\author{
Universidade de São Paulo \\ Instituto de Astronomia, Geofísica e Ciências Atmosféricas
}

Departamento de Astronomia

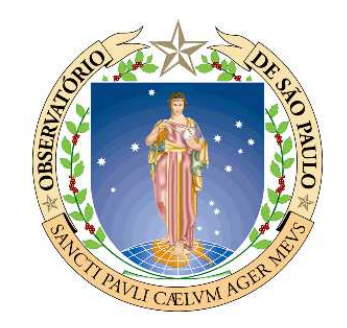

Tiago Mendes de Almeida

\title{
A ORIGEM DO CARBONO NO UNIVERSO - INSIGHTS A PARTIR DE OBSERVAÇÕES DE ESTRELAS POBRES EM METAIS NAS NUVENS DE MAGALHÃES
}

São Paulo 



\section{A ORIGEM DO CARBONO NO UNIVERSO - INSIGHTS A PARTIR DE OBSERVAÇÕES DE ESTRELAS POBRES EM METAIS NAS NUVENS DE MAGALHÃES}

Dissertação apresentada ao Departamento de Astronomia do Instituto de Astronomia, Geofísica e Ciências Atmosféricas da Universidade de São Paulo como parte dos requisitos para a obtenção do título de Mestre em Ciências

Área de Concentração: Astronomia

Orientadora: Prof. ${ }^{a}$ Dr. ${ }^{a}$ Silvia Rossi

São Paulo 

À Almerinda 



\section{Agradecimentos}

Ao meu pai, meu suporte em tempo integral;

Ao meu irmão e família, apoiadores mesmo geograficamente distantes;

A Alciana, minha companheira e amiga completa;

À Prof. Dr. Silvia Rossi, orientadora e mãe nas horas vagas;

A Vinicius Moris Placco, pelo apoio ao longo do andamento deste projeto;

A Alessandro Moisés, pelos ótimos diálogos acompanhados de bons cafés;

Aos pesquisadores Deonísio, Mennickent, Diaz, Masseron, Plez, Beers, Sivarani, pelo suporte técnico e científico;

À Prof. Dr. Barbuy;

À equipe do setor de informática;

Às secretárias do Departamento de Astronomia;

À FAPESP, pelo apoio financeiro, sob o projeto $n^{o}:$ 2006/58659-4;

À Capes e seus auxílio via PROEX;

Ao IAG e toda sua comunidade ${ }^{1}$;

A Maykon, Bira e amigos de São Vicente;

A todos que, embora não tenham sido citados, foram fundamentais para o progresso desta pesquisa.

\footnotetext{
${ }^{1}$ Esta dissertação foi escrita em LATEX com a classe IAGTESE, para teses e dissertações do IAG.
} 

"Em algum ponto perdido deste universo, cujo clarão se estende a inúmeros sistemas solares, houve, uma vez, um astro sobre o qual animais inteligentes inventaram o conhecimento. Foi o instante da maior mentira e da suprema arrogância da história universal."

Friedrich Nietzsche 



\section{Resumo}

Neste projeto de pesquisa planeja-se obter indícios da correlação entre o conteúdo metálico estelar deduzido para a Via Láctea e os indíces metálicos obtidos para as $\mathrm{Nu}$ vens de Magalhães. O ponto de apoio para tal comparação é que cada encontro deixará importantes traços na eficiência de formação dos membros do tripleto. À medida que os encontros dependem da história dinâmica, suas "impressões digitais" deixadas nos conteúdos estelares colocam importantes limitações na história dinâmica e vice-versa.

Para tanto, são utilizados os dados espectroscópicos já obtidos com o telescópio Magellan Clay, para uma amostra contendo 28 estrelas ricas em carbono encontradas nas Nuvens de Magalhães. A quantidade de carbono bem como a existência ou não de binaridade nas estrelas da amostra são indícios das possíveis fontes da sobreabundância do carbono.

A caracterização da amostra é essencial para o estudo da relação entre duas populações estelares aparentemente distintas: a de estrelas de carbono e a de estrelas pobres em metais enriquecidas em carbono. Para tanto, são utilizados catálogos de espectros assim como critérios fotométricos. A descoberta de alguma relação entre as duas populações pode ajudar a esclarecer o problema dos processos de enriquecimento de carbono, notado nas atmosferas estelares.

São estudadas as particularidades de alguns dos objetos, como variabilidade, linhas de

emissão e binaridade. É feita a busca por parâmetros atmosféricos, apesar da dificuldade que resulta da inexistência de grades de modelos de atmosferas compatíveis com a amostra.

A futura determinação dos parâmetros físicos das estrelas que compõem a amostra pode revelar detalhes sobre a história de formação estelar dos objetos das Nuvens e, portanto, vincular a evolução dessas duas galáxias-satélites à história da Via Láctea. 



\section{Abstract}

This project searches for signs of correlation between metallic stellar content, available for the Milky Way, and the metallicities indices obtained for the Magellanic Clouds. This comparison is supported by the traces on the formation of these three galaxies, that should have been left by each triplet members encounter. Since these crosses depend on the dynamical history, their "fingerprints" left by stellar content can estabilish constraints to the Galactic dynamical history.

Spectroscopic data for a sample of carbon stars, obtained on the Magellanic Telescope, are used in this work. The amount of carbon, as the existence or not of binary stars in this sample, indicates possible sources of this element.

The determination of sample properties is essential for studying the constraints between two stellar populations that are apparently distincts: carbon stars and carbon enhanced metal-poor stars. To do this, spectral catalogues and photometric criteria are used. Finding the correlations between both populations will bring some light to the unknown carbon enrichment processes that occured at the stellar atmospheres.

Variability, emission lines, and binarity are studied for the sample. Stellar parameters are discussed, although there is no method applicable to this sample.

By estimating the physical parameters of the stars presented in this sample and by

analysing their carbon abundances, one can provide hints of the star formation history of objects in the Magellanic Clouds and therefore constraint the evolution of these satellitegalaxies to the Milky Way. 



\section{Lista de Figuras}

1.1 Grande e Pequena Nuvens de Magalhães (Mike Bessell, MSSSO). . . . . . 33

1.2 Mosaico de 2400 graus quadrados do mapa de intensidade de HI centrado no polo sul celeste (Putman et al., 1998) . . . . . . . . . . . . . . . 34

1.3 Concepção artística de Dallas Parr, onde são representados o Sistema de Magalhães e a Via Láctea. . . . . . . . . . . . . . . . . . . . . . . . . 35

2.1 Espectros e curvas de luz (magnitude I) das estrelas da SMC (1). . . . . . 45

2.2 Espectros e curvas de luz (magnitude I) das estrelas da SMC (2). . . . . . 46

2.3 Espectros e curvas de luz (magnitude I) das estrelas da LMC (1). . . . . . 47

2.4 Espectros e curvas de luz (magnitude I) das estrelas da LMC (2). . . . . . 48

2.5 Espectros e curvas de luz (magnitude I) das estrelas da LMC (3). . . . . . 49

2.6 Distribuição dos objetos pertencentes a amostra na LMC no espaço de coordenadas galácticas. . . . . . . . . . . . . . . 50

2.7 Distribuição dos objetos pertencentes a amostra na SMC no espaço de coordenadas galácticas. . . . . . . . . . . . . . . . . 51

2.8 Comparação entre $\mathrm{V}$ calculado e $\mathrm{V}$ fornecido pela literatura das estrelaspadrão. . . . . . . . . . . . . . . . . . . . .

3.1 Detalhes das semelhanças entre objetos de tipo espectral C-J nas regiões azul e vermelha do espectro. . . . . . . . . . . . . . . . . . . 70

3.2 Detalhes das semelhanças entre objetos de tipo espectral C-N nas regiões azul e vermelha do espectro. . . . . . . . . . . . . . . . . .

3.3 Detalhes das semelhanças entre objetos de tipo espectral C-H na região azul do espectro. . . . . . . . . . . . . . . . . . . 
3.4 Detalhes das semelhanças entre objetos de tipo espectral C-R na região vermelha do espectro. . . . . . . . . . . . . . . . . . . . 72

3.5 Diagrama cor-magnitude $\mathrm{J}_{-} \mathrm{K}_{s}$ vs. $\mathrm{K}_{s}$. . . . . . . . . . . 73

3.6 Diagrama cor-cor $\mathrm{H}-\mathrm{K}_{s}$ vs. J-H. . . . . . . . . . . . . . . . 74

3.7 Destaque da região azul dos espectros dos sistemas binários OGLE J051609.74693517.9 e OGLE J051810.88-692626.5 . . . . . . . . . . . . . . . . 75 


\section{Lista de Tabelas}

1.1 Nomenclatura de estrelas de diferentes metalicidades (Beers e Christlieb,

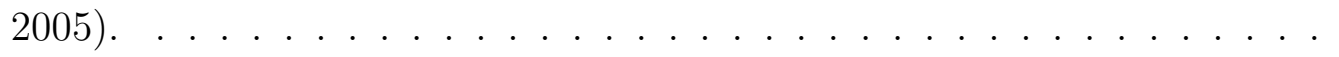

1.2 Definição das subclasses de estrelas pobres em metais (Beers e Christlieb,

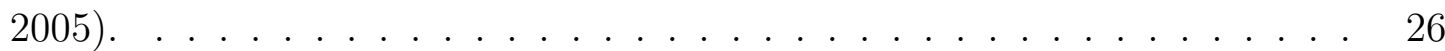

1.3 Informações fundamentais acerca das MC (Westerlund, 1997). . . . . . . . 36

1.4 Informações gerais acerca da estrutura das MC (Westerlund, 1997). . . . . 37

2.1 Log das observações dos 28 objetos da amostra de candidatas a CEMP. . . 43

2.2 Informações sobre a amostra de estrelas das MC. . . . . . . . . . . . . . 53

2.3 Magnitudes absolutas e bolométricas, velocidades radias e tipos espectrais dos objetos da amostra. . . . . . . . . . . . . . . . . 54

2.4 Informações das estrelas consideradas como padrões no cálculo de $\mathrm{V}$ dos objetos da amostra. . . . . . . . . . . . . . . 58 



\section{Sumário}

1. Introdução . . . . . . . . . . . . . . . . . . . . . 21

1.1 Primeiras estrelas . . . . . . . . . . . . . . . . . . . . . . 22

1.2 Estrelas pobres em metais e a sobreabundância de carbono . . . . . . . . . 24

1.3 Estrelas AGB: serão CEMP as estrelas de carbono? . . . . . . . . . . . . . 29

1.4 O Sistema de Magalhães: Nuvens, Corrente e Ponte . . . . . . . . . . . . . 32

1.5 Carbono em estrelas das Nuvens . . . . . . . . . . . . . . . . . . 38

1.6 Objetivos . . . . . . . . . . . . . . . . . . . . . . . . . 39

2. Base de dados . . . . . . . . . . . . . . . . . . . . . . . . . . . . 41

2.1 A amostra de candidatas a estrelas CEMP das Nuvens de Magalhães . . . 41

2.2 Espectroscopia . . . . . . . . . . . . . . . . . . . . . . . . . . 42

2.3 Projeto OGLE e curvas de luz . . . . . . . . . . . . . . . . . . . 44

2.4 Fotometria $\mathrm{IJHK}_{s} \ldots \ldots \ldots \ldots \ldots \ldots$

2.5 Avermelhamento . . . . . . . . . . . . . . . . . . . . . . . . 52

2.6 Magnitudes visuais aparente e absoluta . . . . . . . . . . . . . . . 56

2.7 Estimativa de magnitudes bolométricas . . . . . . . . . . . . . . . . . . . . 59

2.8 Velocidades radiais . . . . . . . . . . . . . . . . . . . . . . . . . . . . . . 59

3. Análise dos dados . . . . . . . . . . . . . . . . . . . . . . 63

3.1 Derivação dos parâmetros atmosféricos de estrelas CEMP . . . . . . . . . . 63

3.1.1 O método de Sivarani et al. (2008) _ . . . . . . . . . . . 65

3.2 Classificação espectral . . . . . . . . . . . . . . . . . . . . . 67

3.2 .1 Sistema MK Revisado . . . . . . . . . . . . . . . . . 67 
3.2.2 Classificação da amostra de candidatas a CEMP . . . . . . . . . . 68

3.3 Identificação de estrelas C por critérios fotométricos . . . . . . . . . . . . . 69

3.4 Resultados . . . . . . . . . . . . . . . . . 73

3.4.1 Tipos espectrais . . . . . . . . . . . . . . . . . 73

3.4.2 Binaridade......................... 75

3.4.3 Fotometria . . . . . . . . . . . . . 76

3.4.4 Curvas de luz . . . . . . . . . . . . . . . . 77

3.4.5 Linhas de emissão em $\mathrm{H} \alpha$. . . . . . . . . . . . . . . . . . . . . . . 78

4. Conclusões e perspectivas . . . . . . . . . . . . . . . . . . . 79

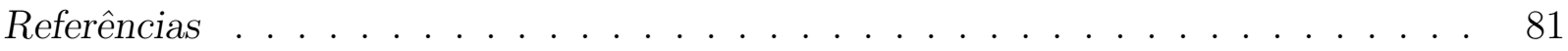




\section{Abreviações e notações}

$[\mathrm{A} / \mathrm{B}]=\log \left(N_{A} / N_{B}\right)_{*}-\log \left(N_{A} / N_{B}\right)_{\odot}$, onde A e B são elementos químicos

$\mu$ - microturbulência

AGB - ramo assimptótico das gigantes (assimptotic giant branch)

$\mathrm{A}_{V}$ - avermelhamento na banda $\mathrm{V}$

BB - Grande Explosão (Big Bang)

C - de carbono

CEH - história de enriquecimento químico (chemical enrichment history)

CEMP - pobre em metais e enriquecida em carbono (carbon-enhanced metal-poor)

CMB - radiação cósmica de fundo de microondas

$\mathrm{dC}$ - estrela de carbono anã (dwarf carbon star)

$\mathrm{E}(\mathrm{B}-\mathrm{V})$ - avermelhamento do índice de cor B-V

EMP - extremamente pobre em metais (extremely metal-poor)

HES - Hamburg/ESO Survey

HMP - hiperpobre em metais (hyper metal-poor)

IMF - função de massa inicial (initial mass function)

ISM - meio interestelar (interstellar medium)

LMC - Grande Nuvem de Magalhães (Large Magellanic Cloud)

$\log g$ - logaritmo da gravidade superficial

$\log g f$ - logaritmo da força de oscilador corrigida pelo fator de Gaunt

LPV - variável de longo período (long period variable)

$\mathrm{M}_{\odot}$ - massa solar

MB - Ponte de Magalhães (Magellanic Bridge)

MC - Nuvens de Magalhães (Magellanic Clouds) 
MDF - função distribuição de metalicidade (metallicity distribution function)

MK93 - Sistema MK Revisado (Keenan, 1993)

MMP - megapobre em metais (mega metal-poor)

MP - pobre em metais (metal-poor)

MS - Corrente de Magalhães (Magellanic Stream)

MW - Via Láctea (Milky Way)

RGB - ramo das gigantes vermelhas (red giant branch)

$\mathrm{R}_{V}$ - extinção na banda $\mathrm{V} \mathrm{S} / \mathrm{R}$ - razão sinal/ruído

SFH - história de formação estelar (star formation history)

SFR - taxa de formação estelar (star formation rate)

SMC - Pequena Nuvem de Magalhães (Small Magellanic Cloud)

SMR - super-rica em metais (super metal-rich)

SBL08 - Sivarani et al. (2008)

$\mathrm{T}_{\text {eff }}$ - temperatura efetiva

UMP - ultrapobre em metais (ultra metal-poor)

$\mathrm{v}_{\text {rad }}$ - velocidade radial

VMP - muito pobre em metais (very metal-poor)

$\mathrm{Z}_{\text {crit }}$ - metalicidade crítica 


\section{Capítulo 1}

\section{Introdução}

Poucos minutos após a Grande Explosão (BB, Big Bang), o Universo se resfriou até atingir o ponto em que prótons e nêutrons puderam existir e se fundir, dando origem aos núcleos atômicos; da união de quatro prótons surgiu o primeiro núcleo de hélio. De forma similar, uma quantidade muito baixa de lítio se formou enquanto os núcleons restantes mantiveram-se na forma de prótons. O único limitante na formação de elementos mais pesados foi a curta existência de condições suficientemente quentes: como o Universo resfriou-se rapidamente, elementos como o carbono, ou mais pesados, não tiveram tempo para serem produzidos. Todos os outros elementos no Universo tiveram que esperar pela formação de estrelas para, em seus interiores, serem produzidos. Assim, qualquer elemento químico presente no Universo atual e que não tenha sido criado logo após o BB foi produzido ao longo da vida de alguma estrela, seja em sua fase quiescente, por fusão nuclear, ou em sua fase explosiva, enquanto supernova, exceção feita aos elementos criados pelo processo de espalação (Reeves et al., 1970).

O papel das estrelas na produção dos elementos químicos tem variado com a idade do Universo. Quando se trata do tempo de vida de uma estrela, a massa é a propriedade mais importante, seguida da composição química. Esta ordem também se mantém na estrutura estelar. Como se sabe, a presença de metais varia com a idade da estrela: as antigas possuem menor quantidade de metais $^{1}$ se comparadas às jovens. Tal distinção se deve ao fato de as estrelas terem se formado em ambientes quimicamente distintos, os quais foram responsáveis pela composição química. É nesse contexto que se insere a procura pelas estrelas mais antigas do Universo, cuja observação pode desvelar informações ainda

\footnotetext{
${ }^{1}$ Embora não esteja de acordo com as definições da Química, em Astronomia são chamados metais os elementos químicos diferentes de $\mathrm{H}, \mathrm{He}, \mathrm{Li}, \mathrm{Be}$ e B.
} 
obscuras sobre o interior estelar, sua evolução e o Universo primordial.

\subsection{Primeiras estrelas}

Desde as primeiras ideias tentadoras de Schwarzschild e Spitzer (1953), a busca pelas primeiras estrelas tem fascinado astrônomos. Atualmente, o assunto tem despertado cada vez mais interesse, tanto sob uma perspectiva teórica quanto observacional.

A População III (Bromm e Larson, 2004), nome dado à geração das primeiras estrelas de acordo com o esquema extendido de classificação estelar do Universo local (Baade, 1944), formada a partir do gás primordial deixado pelo Big Bang e composto somente por $\mathrm{H}$, He e Li, marcou uma transição crucial no final da Idade das Trevas: o Universo tranformou-se de simples e homogêneo para complexo e altamente estruturado. Essa primeira geração estelar se formou sob condições muito mais simples do que as encontradas atualmente nos complexos ambientes das nuvens moleculares. No entanto, a situação subsequente rapidamente se tornou mais complicada devido não somente ao modo como o material ejetado enriquecido em metais se misturou com o gás nas vizinhanças das primeiras regiões de formação estelar (feedback químico), mas também ao grande número de fótons introduzidos no meio interestelar por essas estrelas (feedback radiativo).

A transição de estrelas primordiais às atuais é de grande interesse para o conhecimento da história das primeiras galáxias e da Via Láctea (MW, Milky Way). Teorias preveem que a história da formação estelar (SFH, star formation history) sofreu, em algum instante, uma mudança crítica e, assim, a função de massa inicial (IMF, initial mass function) se deslocou de um domínio de estrelas jovens de alta massa para outro constituído por estrelas de baixa massa (Omukai e Palla, 2001). Sabe-se que a formação de estrelas em gás de metalicidade nula desfavorece a criação de estrelas de baixa massa devido à insuficiência de processos de resfriamento (Bromm et al., 2002). Resultados de simulações numéricas de colapsos e fragmentação de nuvens de gás primordial sugerem que as primeiras estrelas tenham sido muito massivas, com massas típicas de $\mathrm{M}_{*} \gtrsim 100 \mathrm{M}_{\odot}{ }^{2}$ (Bromm et al., 1999; Nakamura e Umemura, 2001). Em contraste com o modo de formação das estrelas massivas de População III em altos redshifts, a observação da fragmentação de nuvens favorece estrelas com massas abaixo da solar no Universo atual. Tal previsão sustenta uma IMF primordial

\footnotetext{
${ }^{2} \mathrm{M}_{\odot}$ significa massa solar e representa uma massa igual a $1,988.10^{30} \mathrm{~kg}$.
} 
bimodal, com picos em 10 e $100 \mathrm{M}_{\odot}$ (Uehara e Inutsuka, 2000; Nakamura e Umemura, 2002).

No entanto, ainda não é claro como a época de formação de estrelas de População III se encerrou. Desde os estudos de Yoshii e Sabano (1980), existe a expectativa de que a metalicidade do gás tenha desempenhado um importante papel na transição entre as Populações III e II; espera-se que diferenças entre os modos de formação estelar dessas duas populações dependam da natureza das primeiras explosões de supernova e, em particular, de como se deu o feedback químico e radiativo. Uma hipótese é que essa transição tenha sido modulada pela radiação cósmica de fundo em microondas (CMB, cosmic microwave background) e por isso tenha sido gradual, envolvendo estrelas de massa intermediária (População II.5) em redshifts intermediários (Mackey et al., 2003; Tumlinson, 2007). Essa população intermediária pode ter dado origem a supernovas menos intensas, ideia frequentemente invocada para explicar os padrões de abundância observados em estrelas pobres em metais (MP, metal-poor; Umeda e Nomoto, 2002, 2003). O conceito de metalicidade crítica $\left(\mathrm{Z}_{\text {crit }}\right.$, onde $\mathrm{Z}$ denota a fração de massa referente a todos os elementos pesados; Omukai, 2000; Schneider et al., 2002, 2003) tem sido utilizado com frequência na definição da População III. Embora não seja necessário um único valor para $\mathrm{Z}_{c r i t}$, este tem sido calculado para condições interestelares típicas $\left(\mathrm{T}=200 \mathrm{~K}, \mathrm{n}=10^{4} \mathrm{~cm}^{-3}\right.$; Rees 1976) e utilizado em modelos mais detalhados.

Em princípio, as estrelas de População III poderiam ter continuado a se formar até épocas recentes, contanto que bolsões de gás suficientemente pobres em metais tenham sido preservados durante a evolução do Universo. Tal cenário pode ter ocorrido no caso de existirem halos que (i) ganharam seu gás de regiões não poluídas por outflows provenientes de galáxias próximas e com estrelas em formação, ou (ii) possuam gás onde a formação de estrelas nunca existiu ou foi coibida (Tornatore et al., 2007).

Entretanto, apesar de todo o empenho até então, a procura observacional por uma estrela legítima que tenha pertencido à primeiríssima geração ou de metalicidade nula tem fracassado, fazendo com que muitos não acreditem em sua existência. Por outro lado, tendo em vista as recentes previsões teóricas de que a maior parte da População III foi muito massiva, com vidas típicas de aproximadamente $10^{6}$ anos, não encontrar uma representante de População III deixou de ser surpreendente, pois tal geração pode ter 
desaparecido há muito tempo (Hernandez e Ferrara, 2001). Existe também a possibilidade do gás primordial ter sido homogeneamente contaminado por metais provenientes das explosões de supernovas (Mori et al., 2002).

Todavia, acredita-se que restem ainda relíquias do final da Era das Trevas: as estrelas MP, representantes da População II extrema.

\subsection{Estrelas pobres em metais e a sobreabundância de carbono}

As estrelas MP observadas hoje em dia constituem o equivalente local do Universo em alto redshift. Por serem representantes de População II, pertencem a gerações estelares que se formaram a partir de um gás com metalicidade não-nula. Sabe-se há muito tempo que as estrelas MP preservam a assinatura das primeiras estrelas em seus padrões de abundância de elementos químicos (Bond, 1981; Beers et al., 1992). Esses objetos guardam em suas atmosferas informação sobre a composição química das nuvens-mãe. As estrelas MP fornecem, portanto, vestígios sobre os tempos mais remotos do Universo. Em particular, padrões de abundâncias fornecem detalhes acerca da formação e evolução dos elementos químicos e dos processos envolvidos em sua nucleossíntese. Tais informações possuem valor inestimável para a compreensão da evolução química e formação de galáxias, fornecendo vínculos observacionais sobre a natureza das primeiras estrelas e supernovas, sendo de fundamental importância para inúmeros trabalhos teóricos. Diante da sua capacidade de armazenar informações sobre os sítios de formação estelar mais antigos, as estrelas MP constituem uma alternativa encorajadora na investigação do Universo remoto.

O termo pobre em metal não necessariamente reflete o conteúdo total de elementos químicos pesados da estrela mas sim a abundância do ferro, utilizada para definir metalicidade. Os valores de abundância de ferro, bem como a classificação que indica quão pobre em metal é uma estrela, são obtidos em comparação aos valores solares, uma vez que o Sol é considerado uma estrela de sequência principal de baixa massa típica da IMF atual, além de ter suas abundâncias elementais muito bem determinadas (Lodders, 2003; Grevesse et al., 2007). A notação mais utilizada para metalicidade é $[\mathrm{Fe} / \mathrm{H}]^{3}$.

Tendo em vista as inúmeras classificações utilizadas na descrição da deficiência em

\footnotetext{
${ }^{3}[\mathrm{~A} / \mathrm{B}]=\log \left(N_{A} / N_{B}\right)_{*}-\log \left(N_{A} / N_{B}\right)_{\odot}$, onde A e B são elementos químicos, é a notação adotada nesse trabalho para representar abundâncias de metais.
} 
Tabela 1.1 - Nomenclatura de estrelas de diferentes metalicidades (Beers e Christlieb, 2005).

\begin{tabular}{ccc}
\hline \hline$[\mathrm{Fe} / \mathrm{H}]$ & Termo & Sigla \\
\hline$>+0,5$ & Super-rica em metais (super metal-rich) & SMR \\
$\sim 0,0$ & Solar & - \\
$<-1,0$ & Pobre em metais (metal-poor $)$ & $\mathrm{MP}$ \\
$<-2,0$ & Muito pobre em metais (very metal-poor) & $\mathrm{VMP}$ \\
$<-3,0$ & Extremamente pobre em metais (extremely metal-poor) & $\mathrm{EMP}$ \\
$<-4,0$ & Ultrapobre em metais (ultra metal-poor) & $\mathrm{UMP}$ \\
$<-5,0$ & Hiperpobre em metais (hyper metal-poor) & HMP \\
$<-6,0$ & Megapobre em metais (mega metal-poor) & MMP \\
\hline
\end{tabular}

metais das estrelas, Beers e Christlieb (2005) propuseram padronizar a nomenclatura dos objetos MP. A Tabela 1.1 apresenta a proposta de classificação de estrelas de diferentes metalicidades.

Das três estrelas com menor metalicidade conhecidas atualmente, duas são HMP e uma UMP. A observação das estrelas HE 0557-4840, com $[\mathrm{Fe} / \mathrm{H}]=-4,8$ (Norris et al., 2007), HE0107-5240, com $[\mathrm{Fe} / \mathrm{H}]=-5,3$ (Christlieb et al., 2002, 2004) e HE1327-2326, com $[\mathrm{Fe} / \mathrm{H}]=-5,4$ (Frebel et al., 2005; Aoki et al., 2006) reflete um desenvolvimento surpreendente no estudo em andamento dos "fósseis" de estrelas antigas, uma vez que tais objetos surgiram além de um intervalo aparente na distribuição de metalicidade das estrelas do halo galático $(-4<[\mathrm{Fe} / \mathrm{H}]<-3)$, que até pouco tempo se acreditava ser um limite inferior na metalicidade das estrelas pobres em metais da Galáxia (Carr, 1987; Oey, 2003).

Ao longo das últimas décadas surgiram inúmeras subclasses de estrelas deficientes em metais. Algumas delas têm sido frequentemente associadas a sítios astrofísicos específicos de produção de elementos pesados. Beers e Christlieb (2005) propuseram uma taxionomia para as categorias mais reconhecidas. Ainda não é claro se todas as divisões propostas são fisicamente relevantes uma vez que elas estão relacionadas a diferentes histórias de nucleossíntese. Além disso, em alguns casos há indícios da existência de uma coleção de parâmetros relevantes, como no caso do enriquecimento por elementos produzidos por processos de captura de nêutrons. Isso faz com que a taxionomia baseada em subclasses discretas de estrelas pobres em metais deva ser considerada apenas como uma primeira 
Tabela 1.2 - Definição das subclasses de estrelas pobres em metais (Beers e Christlieb, 2005).

\begin{tabular}{|c|c|}
\hline & Estrelas enriquecidas em elementos $r$ e $s$ \\
\hline Sigla & Critério de classificação \\
\hline $\mathrm{r}-\mathrm{I}$ & $+0,5 \leq[\mathrm{Eu} / \mathrm{Fe}] \leq+1,0$ e $[\mathrm{Ba} / \mathrm{Eu}]<0$ \\
\hline r-II & {$[\mathrm{Eu} / \mathrm{Fe}]>+1,0$ e $[\mathrm{Ba} / \mathrm{Eu}]<0$} \\
\hline $\mathrm{s}$ & {$[\mathrm{Ba} / \mathrm{Fe}]>+1,0$ e $[\mathrm{Ba} / \mathrm{Eu}]>+0,5$} \\
\hline \multirow[t]{2}{*}{$\mathrm{r} / \mathrm{s}$} & $0,0<[\mathrm{Ba} / \mathrm{Eu}]<+0,5$ \\
\hline & Estrelas enriquecidas em carbono \\
\hline CEMP & {$[\mathrm{C} / \mathrm{Fe}]>+1,0$} \\
\hline CEMP-r & {$[\mathrm{C} / \mathrm{Fe}]>+1,0$ e $[\mathrm{Eu} / \mathrm{Fe}]>+1,0$} \\
\hline CEMP-s & {$[\mathrm{C} / \mathrm{Fe}]>+1,0,[\mathrm{Ba} / \mathrm{Fe}]>+1,0$ e $[\mathrm{Ba} / \mathrm{Eu}]>+0,5$} \\
\hline CEMP-r/s & {$[\mathrm{C} / \mathrm{Fe}]>+1,0$ e $0,0<[\mathrm{Ba} / \mathrm{Eu}]<+0,5$} \\
\hline CEMP-no & {$[\mathrm{C} / \mathrm{Fe}]>+1,0$ e $[\mathrm{Ba} / \mathrm{Fe}]<0$} \\
\hline
\end{tabular}

aproximação. Apesar de que essa nomenclatura certamente necessitará de uma revisão no futuro, acredita-se que a sugestão de Beers e Christlieb ainda é útil por caracterizar as propriedades da diversidade de estrelas deficientes em metais. Na Tabela 1.2 são apresentadas as definições das subclasses de estrelas pobres em metais. Trabalhos como os de Masseron et al. (2009) propõem uma classificação alternativa com base em novas evidências observacionais acerca dos padrões de abundâncias das estrelas MP.

Dentre as estrelas pobres em metais enriquecidas por elementos produzidos via processos $r$ e $s$ de captura de nêutrons ${ }^{4}$ (Burbidge et al., 1957), as maiores subclasses são as de estrelas $r$, estrelas $s$ e estrelas $r / s$, caracterizadas pela alta abundância de elementos produzidos por ambos os processos. Há a divisão de estrelas $r$ em uma subclasse $r$-I, caracterizada pelo baixo enriquecimento em elementos $r$ (produzidos pelo processo $r$ ), e outra $r$-II, onde esse enriquecimento é relativamente maior. Definições quantitativas são apresentadas na Tabela 1.2. O Eu é usado como referência dos elementos $r$ no material contido no sistema solar pois sua abundância é a mais fácil de ser medida no espectro óptico das estrelas MP. A condição $[\mathrm{Ba} / \mathrm{Eu}]<0$ é incluída nas definições uma vez que o Eu

\footnotetext{
${ }^{4} r$ e $s$ são as abreviações de rapid (rápido) e slow (lento) respectivamente, fazendo referência à escala de tempo da captura de nêutrons com relação ao decaimento beta.
} 
é também produzido pelo processo $s$, de forma que o Ba, produzido principalmente via processo $s$, atua como um discriminante na classificação.

Atualmente, tem-se dado destaque especial a uma classe de estrelas MP: a das estrelas que apresentam excesso em sua abundância de carbono.

Muito do que se sabe hoje acerca da sobreabundância de carbono nas estrelas MP se deve ao esforço empenhado na construção de uma base de dados sobre estrelas pobres em metais. Nesse sentido, merece destaque a contribuição dos surveys HK (Beers et al., 1985, 1992) e Hamburg/ESO (HES; Reimers 1990; Wisotzki et al. 2000). Ambos tiveram como características amplas aberturas, utilização da placas fotográficas combinadas com a técnica de prisma-objetivo, baixa resolução espectroscópica e a cobertura de vários graus e regiões profundas do céu. Juntos resultaram em numerosas amostras de estrelas MP da Galáxia e posterior identificação de estrelas pobres em metais enriquecidas em carbono (CEMP, carbon-enhanced metal-poor).

O survey HK cobriu áreas de 2800 e 4100 graus quadrados no hemisférios norte e sul, respectivamente. Os espectros das fontes captadas pelas placas fotográficas tiveram sua banda de passagem limitada em $150 \AA ̊$ e centrada em $\lambda 3950$, região onde se encontram as linhas H e K do CaII. A inspeção visual das placas permitiu a seleção de cerca de 10000 objetos candidatos a MP, que posteriormente tiveram seus espectros de média resolução obtidos em diferentes telescópios para assim terem suas metalicidades determinadas. Os candidatos a MP foram identificados com base na força da linha K do CaII.

O survey HES cobriu uma área de 8225 graus quadrados do hemisfério sul na procura por quasares brilhantes. A busca por estrelas MP em sua base de dados, empenhada por Christlieb (2003), possibilitou o aumento do número de estrelas EMP em quase uma ordem de grandeza com relação ao survey HK. Isso porque o HES atingiu magnitudes mais fracas, $10 \lesssim \mathrm{B} \lesssim 17,5$ em comparação ao intervalo $10 \lesssim \mathrm{B} \lesssim 15,5$ do survey $\mathrm{HK}$. Suas placas fotográficas foram digitalizadas, o que permitiu a classificação quantitativa dos objetos. As candidatas foram selecionadas pelo índice de linha KP, uma indicação da força da linha K do CaII medido a partir do espectro de prisma-objetivo digitalizado. A calibração em fluxo das placas fotográficas permitiu o uso do índice de cor B-V na tentativa de se corrigir um viés de temperatura na seleção das estrelas candidatas a MP, estabelecendo assim mais uma vantagem do HES sobre o HK. A posterior obtenção de espectroscopia em média 
resolução de objetos selecionados com base em uma série de critérios foi apreciada, assim como no survey HK.

Observações recentes de candidatas a estrelas VMP selecionadas a partir dos surveys HK e HES revelaram que aproximadamente $20 \%$ das estrelas com $[\mathrm{Fe} / \mathrm{H}] \leq-2,0$ apresentam sobreabundâncias de carbono em relação ao ferro $([\mathrm{C} / \mathrm{Fe}]>+1$; Cohen et al. 2005; Lucatello et al. 2006). Essa fração é ainda maior nas estrelas com metalicidades mais baixas, o que pode ser constatado nas três estrelas com $[\mathrm{Fe} / \mathrm{H}]<-4$, as quais são extremamente ricas em carbono. Já a porcentagem de estrelas no disco com metalicidades próximas a solar que exibem sobreabundância de carbono não é muito maior que a unidade (Norris et al., 1997).

O fenômeno de enriquecimento em carbono em estrelas é facilmente notado pela presença em seus espectros de fortes bandas em absorções em torno de $\lambda 4300$ (banda G do $\mathrm{CH}$ ) e de absorções por moléculas como $\mathrm{CN}$ e $\mathrm{C}_{2}$ (características mais proeminentes em estrelas com temperaturas efetivas mais baixas). A tendência da razão [C/Fe] crescer com $[\mathrm{Fe} / \mathrm{H}]$ nas estrelas que apresentam deficiência em metais e sobreabundância de carbono em relação ao ferro foi apontada observacionalmente, pela primeira vez, por Rossi et al. (1999). Sabe-se também que, ao longo do intervalo de metalicidades $-4,0<[\mathrm{Fe} / \mathrm{H}]<$ -2,0, existe um limite superior de enriquecimento dentre as estrelas CEMP em $[\mathrm{C} / \mathrm{H}] \sim 0,0$ (Aoki et al., 2008). A fração de estrelas CEMP pode ainda aumentar com a distância ao plano Galáctico (Frebel et al., 2006).

Uma fração significativa de estrelas CEMP (aproximadamente 70-80\%, de acordo com Aoki et al., 2007) apresenta sobreabundância também em elementos s, produzidos em estrelas do ramo assimptótico das gigantes (AGB, assimptotic giant branch) como Ba e Pb. Além disso, algumas estrelas CEMP são enriquecidas em elementos $r$, muito provavelmente provenientes de explosões de supernovas. Esse tipo de característica sugeriu uma divisão bastante conveniente das estrelas CEMP em estrelas enriquecidas somente por elementos $r$ (CEMP-r), somente por elementos $s$ (CEMP-s), por elementos $r$ e $s$ (CEMP-r/s) ou que não apresentam enriquecimento em elementos $s$ (CEMP-no). As subclasses de estrelas CEMP são apresentadas na Tabela 1.2.

A origem da sobreabundância de carbono e de elementos $r$ e $s$ em estrelas MP ainda não é clara. No entanto, é pouco provável que objetos com padrões de abundância tão 
distintos entre si tenham se formado sob as mesmas condições. Atualmente há três cenários propostos para explicar a origem das sobreabundâncias em estrelas MP (Beers e Christlieb, 2005): (i) um mecanismo de produção desses elementos em estrelas massivas primordiais, (ii) produção interna em estrelas de baixa massa e baixíssima metalicidade e (iii) produção de carbono por estrelas de massa intermediária, sintetizado durante sua fase de AGB, seguida de transferência do material enriquecido para uma companheira de baixa massa $\left(\mathrm{M} \sim 0,8 \mathrm{M}_{\odot}\right)$ que tenha sobrevivido até os dias atuais.

\subsection{Estrelas AGB: serão CEMP as estrelas de carbono?}

Assim como as estrelas MP, estrelas em estágios evolutivos avançados como o AGB também apresentam excesso de carbono em suas atmosferas. No entanto, sua correlação com estrelas CEMP ainda não está bem estabelecida.

O AGB é o estágio final de evolução de estrelas de massas intermediária e baixa (1 $\leq \mathrm{M}_{\odot} \leq 8$; Herwig, 2005). A principal característica dessa fase é a fusão de hidrogênio em hélio em finas camadas que envolvem o caroço degenerado de carbono ou oxigênio, produzidos a partir da fusão de átomos de hélio. Seus pulsos termonucleares recorrentes, que provocam inúmeros eventos de mistura convectiva da composição química, garantem a diversidade dos processos de reações nucleares no interior estelar.

As estrelas AGB desempenham um papel importante no estudo da nucleossíntese estelar e na origem dos elementos químicos. Por ejetarem para o meio interestelar material enriquecido em elementos pesados (principalmente na fase pós-AGB), sua contribuição para a evolução química galáctica é fundamental. Dotadas de alta luminosidade em comparação a outras populações estelares, as AGB são facilmente identificadas observacionalmente, além de serem facilmente encontradas nos catálogos e surveys não só da Galáxia mas também de sistemas estelares externos, como de galáxias-satélites da MW.

O transporte convectivo, responsável pelos episódios de dragagem que misturam material do interior estelar com o da superfície, faz com que estrelas AGB tenham sua fria atmosfera enriquecida (com relação à abundância solar) em carbono e/ou oxigênio, dependendo da massa inicial. Como resultado, a energia irradiada que atravessa a superfície da AGB é afetada pela interação com moléculas desses elementos, originando fortes bandas de absorção molecular nas regiões visível e infravermelha dos espectros das estrelas. 
Devido à variabilidade de brilho, resultado de pulsações em seus interiores, as estrelas AGB são normalmente objeto de estudos como estrelas variáveis de longo período (LPV, long period variables).

Em princípio, as estrelas AGB podem ser separadas em duas classes considerandose suas abundâncias espectrais: em estrelas tipo $\mathrm{O}$ ou $\mathrm{M}$, com $\mathrm{C} / \mathrm{O}<1$, e estrelas de carbono (estrelas $\mathrm{C}$ ), com $\mathrm{C} / \mathrm{O}>1$. O enriquecimento em carbono ou sua ausência se deve à eficiência da terceira dragagem, episódio causado pelos pulsos térmicos em que ${ }^{12} \mathrm{C}$ é transportado do interior à superfície, levando ao aumento da razão $\mathrm{C} / \mathrm{O}$. Os pulsos térmicos provocam também a perda de massa e a variação de luminosidade em períodos de 100 dias ou maiores, com diferenças nas amplitudes pico-a-pico de até algumas magnitudes no visível.

As estrelas C têm como principal característica espectral fortes bandas de absorção por moléculas de $\mathrm{C}_{2}$, principalmente em $\lambda 5160$ (Wallerstein e Knapp, 1998). Suas temperaturas e luminosidades correspondem aos tipos espectrais $\mathrm{G}$ tardio, $\mathrm{K}$ e M.

O estudo das estrelas $\mathrm{C}$ vêm se desenvolvendo de forma independente desde o século XIX. Elas foram descobertas e estudadas pela primeira vez pelo Padre Angelo Secchi em Roma no ano de 1866. Foi no catálogo de Henry Draper (Cannon e Pickering, 1918) onde surgiu a classificação dessas estrelas em tipos $\mathrm{N}$ e R. A falta de informação acerca da temperatura das estrelas levou Keenan e Morgan (1941) a reclassificarem-nas. Esse novo sistema, conhecido como Classificação C, foi ligeiramente modificado por Yamashita (1972). Com o passar dos tempos, evidências físicas esclareceram que os tipos $\mathrm{N}$ e $\mathrm{R}$ correspondiam, na verdade, a populações distintas (Sanford, 1944; Dean, 1976). A partir do conhecimento acerca das estrelas $\mathrm{C}$ desenvolvido até então, a Classificação C foi reorganizada de forma mais flexível no chamado Sistema MK Revisado (Keenan, 1993, daqui em diante, MK93). Além de manter as distinções entre estrelas $\mathrm{R}$ e $\mathrm{N}$ (C-R e C-N), o novo sistema passou a incorporar os tipos referentes a estrelas com baixa razão ${ }^{12} \mathrm{C} /{ }^{13} \mathrm{C}(\mathrm{C}-\mathrm{J})$, com deficiência em hidrogênio (C-Hd) e estrelas com excesso de CH (C-H). Barnbaum (1994) e Barnbaum et al. (1996) compilaram atlas espectrais de alta e média resolução que ilustram as características do Sistema MK93. Portanto, há na literatura uma densa base de dados acerca das estrelas C, tanto espectroscópica quanto fotométrica, disponibilizadas em inúmeros surveys não apenas da Galáxia (Downes et al., 2004) mas também da Pequena (Rebeirot et al., 1993; 
Morgan e Hatzidimitriou, 1995) e da Grande Nuvem de Magalhães (Blanco e McCarthy, 1983; Kontizas et al., 2001).

Estrelas AGB observadas nas Nuvens de Magalhães (MC, Magellanic Clouds) e na MW indicam que a maioria das estrelas $\mathrm{C}$ reside no intervalo de luminosidades $-5<\mathrm{M}_{b o l}<$ -3 (Westerlund et al., 1991). O fato de não se encontrar tais estrelas com $\mathrm{M}_{b o l}<-7$ (Paczynski, 1971) sugere que estrelas com massas intermediárias possuam um processo de produção de ${ }^{14} \mathrm{~N}$ a partir do consumo de ${ }^{12} \mathrm{C}$, a chamada queima na base do envoltório convectivo (HBB, hot bottom burning).

É importante ressaltar a diferença entre as estrelas $\mathrm{C}$ e as estrelas CEMP consideradas neste trabalho. Enquanto as primeiras são definidas por apresentarem bandas de $\mathrm{C}_{2}$ na região visível de seus espectros, as CEMP são caracterizadas pela razão $[\mathrm{C} / \mathrm{Fe}]>1$. Como consequência dessas definições, nem todas as estrelas C são pobres em metais, apesar da comunhão dessas características ser relativamente comum.

Acredita-se que a maioria das estrelas C (ao menos as gigantes) constitua uma fase tardia da evolução das estrelas AGB. Os processos de transporte de material enriquecido em carbono de regiões internas para externas em estrelas nessa fase (por exemplo, terceira dragagem) pode estar correlacionado de alguma forma com a escassez de elementos pesados acompanhado do enriquecimento em C nas estrelas de População II extrema. Esse é o principal incentivo para a busca de estrelas CEMP dentre a numerosa população de estrelas C das MC.

Como a origem da sobreabundância do carbono estelar ainda não está bem estabelecida, os parâmetros que discriminam as estrelas CEMP das C não são bem conhecidos. A dificuldade em se distinguir as duas populações aumenta quando são consideradas estrelas com baixa temperatura efetiva, como de tipo esepctral M e mais tardios. Mais obscura ainda é a identificação de objetos sob os efeitos de enriquecimento extrínseco e intrínseco simultâneos - ou seja, de binaridade acompanhada da troca de material enriquecido em carbono e de enriquecimento da atmosfera por dragagem do conteúdo interno. A descoberta de sistemas binários com troca de material enriquecido em carbono (McClure e Woodsworth, 1990), como a estrela de carbono anã (dC, dwarf carbon star) G77-61 (Dahn et al., 1977; Plez e Cohen, 2005), as estrelas de Ba e as de tipo espectral C-H (McClure, 1984), tornou fundamental a investigação no sentido de dintinguir esses dois efeitos. 
O método que vem sendo empregado para se desvendar essa ambiguidade é (i) a identificação de elementos químicos representativos do estágio evolutivo das estrelas e (ii) determinação das classes de luminosidade das estrelas, o que depende do conhecimento da energia total por elas emitida. Nesse sentido, as MC, por serem mais pobres em metais que a MW e por possuírem uma população numerosa de estrelas C, são um valioso laboratório para a investigação da origem da sobreabundância de carbono no Universo local.

\subsection{O Sistema de Magalhães: Nuvens, Corrente e Ponte}

As MC (Figura 1.1) são duas galáxias irregulares que orbitam a MW. Ambas localizamse ao sul do Equador Celeste; a Grande Nuvem (LMC, Large Magellanic Cloud) está centrada em aproximadamente $\alpha=5^{h} 24^{m}, \delta=-69^{\circ} 45^{\prime}$ (J2000), enquanto a Pequena Nuvem (SMC, Small Magellanic Cloud) tem seu centro em $\alpha=0^{h} 52^{m}, \delta=-72^{\circ} 50^{\prime}$ (de Vaucouleurs et al., 1976). Devido à sua proximidade (Figura 1.2), as MC são duas das galáxias mais bem estudadas no Universo: a LMC e a SMC estão a distâncias da ordem de 50 e 60 kpc, respectivamente (Freedman et al., 2001; Hilditch et al., 2005). Nas Tabelas 1.3 e 1.4 são apresentadas informações gerais sobre as MC. Sabe-se que a SMC é mais pobre em metais que a LMC. Outras de suas propriedades que merecem destaque são sua baixa razão entre massa de poeira e de gás, altas razões de abundâncias $[\mathrm{O} / \mathrm{C}]$ e $[\mathrm{O} / \mathrm{N}]$, alto valor da razão entre número de hidrogênio atômico e molecular, além do intenso campo de radição no ultravioleta. As Nuvens, que são muito menores que a MW, são caracterizadas pelo baixo grau de processamento nuclear quando comparadas à Galáxia. No entanto, atualmente elas vêm passando por um episódio de formação estelar (Carlson et al., 2007).

$\mathrm{Na}$ região que se estende das Nuvens ao polo sul celeste, abrangendo $100^{\circ}$, localiza-se a chamada Corrente de Magalhães (MS, Magellanic Stream), uma estrutura de gás de HI de aproximadamente $10^{\circ}$ de largura. Ela foi observada pela primeira vez por Dieter (1965) que, vasculhando os polos Galácticos com a antena de 18m de Harvard, detectou altas velocidades radiais próximas ao polo sul, sem contrapartida no polo norte. No entanto, naquela ocasião, a MS não havia sido identificada como uma estrutura coerente. Observações subsequentes da região norte da MS, chamada naquela época de "complexo do polo sul", foram feitas por van Kuilenburg (1972) e Wannier e Wrixon (1972). Mathewson et al. (1974) observaram a região sul da MS usando o radiotescópio Parkes de $18 \mathrm{~m}$ e 


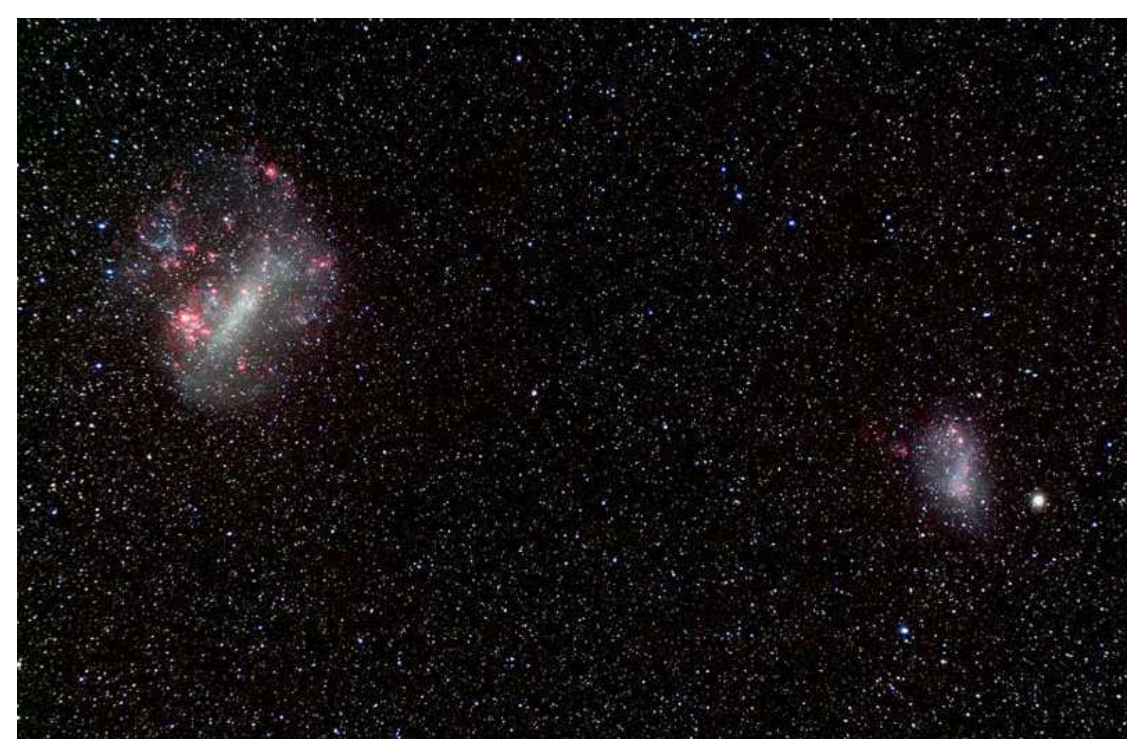

Figura 1.1: Grande (à esquerda) e Pequena (à direita) Nuvens de Magalhães (Mike Bessell, MSSSO).

identificaram a conexão entre o "complexo do polo sul" e as MC, dando assim o nome de MS à conexão.

Entre as Nuvens, há um estrutura formada principalmente por hidrogênio neutro, conhecida como Ponte de Magalhães (MB, Magellanic Bridge). Essa componente, junto com as MC e MS, compõe o Sistema de Magalhães. A Figura 1.3 é uma concepção artística representando a proximidade entre o Sistema e a MW, dando destaque à Corrente.

As Nuvens estão inseridas em um envoltório comum de hidrogênio neutro, uma indicação de que estão ligadas há muito tempo. Desse fato decorre a hipótese, atualmente bem aceita embora não confirmada, de que as Nuvens estão ligadas à Galáxia há pelo menos 7 bilhões de anos (Kallivayalil et al., 2006). Outra hipótese recorrente nos modelos é que a MS acompanha o deslocamento espacial das MC (Lin et al., 1995).

O Sistema de Magalhães possui ainda um papel importante na compreensão de diversas áreas da Astronomia, tais como a escala de distância galáctica que usa a LMC como um ponto-zero (Feast, 1999; Gibson, 2000), a formação de aglomerados estelares e regiões HII, astrofísica de nuvens moleculares e efeitos de microlente no halo Galáctico (Alcock et al., 2000). O Sistema move-se no potencial gravitacional da MW e no plano definido pelo Grupo Local. É de se esperar que a influência da nossa Galáxia seja notada na atual estrutura e cinemática do Sistema (Bekki e Stanimirovic, 2009).

Um dos problemas-chave relacionado às Nuvens é a compreensão de como as interações 


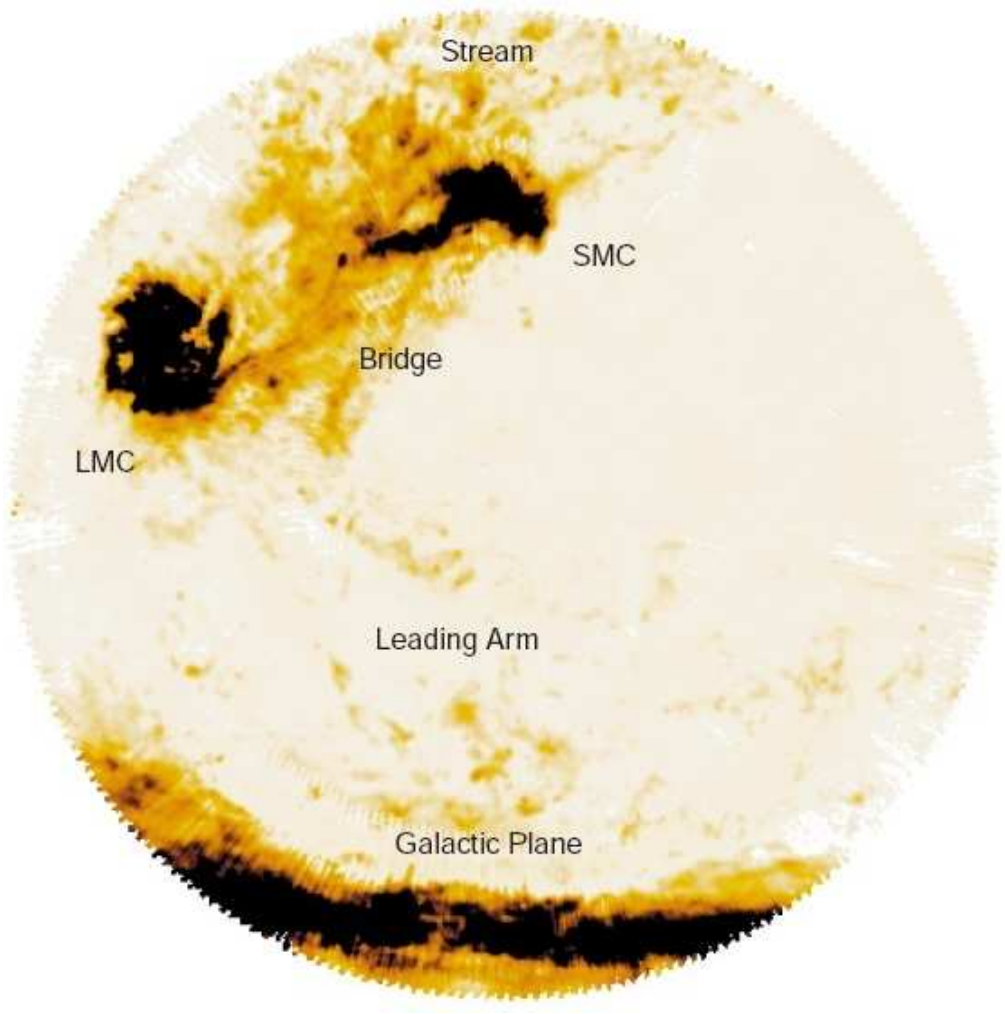

Figura 1.2: Mosaico de 2400 graus quadrados do mapa de intensidade de HI centrado no polo sul celeste. As bordas da imagem delimitam os $-62^{\circ}$ de declinação. Ascenção reta 0h está no topo e cresce no sentido anti-horário (Putman et al., 1998).

dinâmicas do passado entre as MC e MW puderam influenciar a evolução química e dinâmica da SFH das MC (em especial da SMC; Westerlund, 1997). As propriedades estruturais, cinemáticas e químicas observadas de estrelas, aglomerados e gás na SMC vêm sendo discutidas exaustivamente sob diferentes aspectos, como, por exemplo, sua SFH (Russell e Dopita, 1992; Piatti et al., 2007), massa dinâmica total (Dopita et al., 1985; Stanimirovic et al., 2004) e história de interação com a LMC (Bekki e Chiba, 2007).

Interações entre galáxias, eventos relativamente comuns, são capazes de influenciar propriedades físicas como a morfologia global e a taxa de formação estelar (SFR, star formation rate). Interações dinâmicas entre a SMC, LMC e MW têm sido consideradas como intimamente associadas não apenas com a evolução das nuvens (Yoshizawa e Noguchi, 2003), mas também com a formação da MS e MB (Muller et al., 2004). Como companheiras da MW, é de se esperar que interações de maré desempenhem um papel importante na evolução dinâmica das regiões externas da Galáxia. 


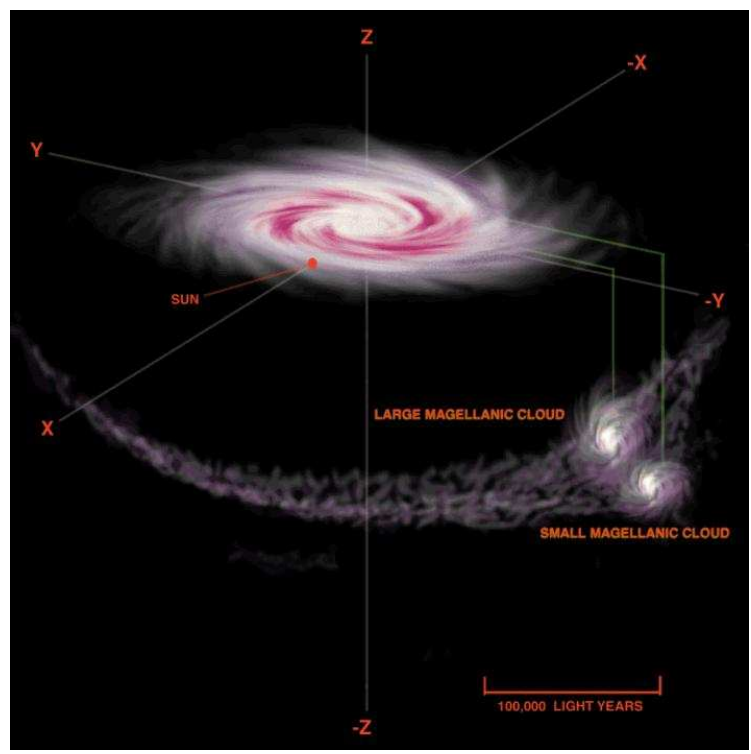

Figura 1.3: Concepção artística de Dallas Parr, onde são representados o Sistema de Magalhães e a MW. É dado destaque à MS.

Há uma série de indícios de efeitos de maré entre as Nuvens e a Galáxia (Putman et al., 1998). A pequena velocidade radial Galactocêntrica das Nuvens indica que suas posições nas órbitas traçadas ao redor da MW são aproximadamente perigalácticas ou apogalácticas. Se aproximadamente apogalácticas, então efeitos de maré podem ter induzido a distorção (warp) no plano Galáctico durante os encontros no passado (Toomre, 1972; Fujimoto e Sofue, 1976). A evidência mais forte de efeitos de maré surgiu das varreduras do céu em busca de nuvens de HI de alta velocidade que revelaram a MS.

O estudo do enriquecimento químico de sistemas estelares consiste em determinar como as abundâncias de diversos elementos evoluem no tempo e variam no espaço, considerando abundâncias determinadas adequadamente e com boa acurácia. O papel das MC na compreensão dos diversos processos de evolução química mostra-se interessante por elas apresentarem baixa metalicidade (próxima à primordial, principalmente quando se trata da SMC), além de terem suas distâncias relativamente bem determinadas e possuírem avermelhamento baixo quando comparado ao do disco Galáctico. As MC apresentam um amplo intervalo de idades e metalicidades, o que aumenta sua importância no estudo do enriquecimento químico de galáxias.

Sabe-se que as histórias de enriquecimento químico (CEH, chemical enrichment history) das Nuvens apresentam padrões distintos, embora ainda um tanto controversas em vários 
Tabela 1.3 - Informações fundamentais acerca das MC (Westerlund, 1997).

\begin{tabular}{lll}
\hline \hline & LMC & SMC \\
\hline Tipo & $\mathrm{SB}(\mathrm{s}) \mathrm{m}^{(1)}$ & $\mathrm{SB}(\mathrm{sm}) \mathrm{mp}{ }^{(1)}$ \\
$\mathrm{l}, \mathrm{b}$ & $280^{\circ}, 5,-32^{\circ}, 9^{(2)}$ & $302^{\circ}, 8,-44^{\circ}, 3^{(2)}$ \\
$\mathrm{m}-\mathrm{M}$ & $18,5 \pm 0,1^{(3)}$ & $18,91 \pm 0,10^{(4)}$ \\
$\mathrm{RV}_{\text {hel }}$ & $262,2 \mathrm{~km} \mathrm{~s}^{-1}{ }^{(5)}$ & $149,3 \mathrm{~km} \mathrm{~s}^{-1}(6)$ \\
Tilt & $33^{\circ}-45^{\circ}$ & $90^{\circ}$
\end{tabular}

\section{Cores e magnitudes integradas}

$\begin{array}{lll}\text { B } & 0,9\left(100 \operatorname{graus}^{2}\right) & 2,9\left(12 \operatorname{graus}^{2}\right) \\ \text { B-V } & 0,5 & 0,4 \\ \text { Brilho Central Superficial B } & 21,2 \mathrm{mag} \mathrm{arcsec}^{-2} & 21,4{\mathrm{mag} \operatorname{arcsec}^{-2}}^{2} \\ \text { B-V } & 0,46 & 0,25\end{array}$

\section{Massas}

Total

H I

H I $(21 \mathrm{~cm})$

$\mathrm{H}_{2}$ (linha de $\mathrm{CO}$ )

Poeira quente (IRAS)

Poeira fria
$2.10^{10} \mathrm{M}_{\odot}$

$\leq 8 \%$ da massa total

$7.10^{8} \mathrm{M}_{\odot}$

$1,4.10^{8} \mathrm{M}_{\odot}$

4-50.10 ${ }^{3} \mathrm{M}_{\odot}$

$8-90.10^{5} \mathrm{M}_{\odot}$
$2.10^{9} \mathrm{M}_{\odot}$

$\sim 30 \%$

$6,5.10^{8} \mathrm{M}_{\odot}$ $3,0.10^{7} \mathrm{M}_{\odot}$ $8-10.10^{4} \mathrm{M}_{\odot}$ $9-100.10^{5} \mathrm{M}_{\odot}$

Notas - A massa da população antiga e com $[\mathrm{Fe} / \mathrm{H}] \leq-1,4$ na LMC é $\sim 6 \%$ da sua massa total (Frogel 1984); a massa da população antiga e com $[\mathrm{Fe} / \mathrm{H}] \sim-1,0 \pm 0,5$, representada pelas LPV, é $\sim 2 \%$ da massa total (Hughes et al., 1991).

A poeira normal média das Nuvens é mais quente que a Galáctica devido ao alto campo de radiação interestelar (Lequeux, 1989). Pequenos grãos podem ter sido destruídos pela radiação UV, abundante na região.

Referências: (1) de Vaucouleurs e Freeman (1972), (2) de Vaucouleurs et al. (1976), (3) Freedman et al. (2001), (4) Hilditch et al. (2005), (5) van der Marel et al. (2002), (6) Hatzidimitriou et al. (1997).

aspectos; a época e a taxa dos principais episódios de formação estelar nas MC ainda são objeto de debate e muitos cenários têm sido propostos. Para a SMC, enquanto Harris e Zaritsky (2004) sugerem três episódios há 400 milhões, 3 e 9 bilhões de anos, Dolphin et al. (2001) favorece uma formação estelar contínua no halo com um episódio dominante entre 5 e 8 bilhões de anos atrás. Em particular, Zaritsky e Harris (2004) defendem que os principais episódios de aumento na formação estelar na SMC foram disparados pelas 
Tabela 1.4 - Informações gerais acerca da estrutura das MC (Westerlund, 1997).

\begin{tabular}{|c|c|c|}
\hline & $\mathrm{LMC}$ & $\mathrm{SMC}$ \\
\hline Disco: & $\begin{array}{l}\text { Possui um disco maior composto por } \\
\text { gás e populações de estrelas jovens e } \\
\text { de idade intermediária }\end{array}$ & $\begin{array}{l}\text { Camadas de HI podem ser vistas em } \\
\text { profundidade; se houver um disco, deve } \\
\text { ser visto na linha de visada. }\end{array}$ \\
\hline Regiões HII & Numerosas & Algumas \\
\hline Emissão $\mathrm{H} \alpha$ difusa & Sim, extendida & Sim, extendida \\
\hline Nuvens moleculares & Numerosas & Algumas, principalmente a nordeste \\
\hline Barra: & $\begin{array}{l}\text { Estrelas vermelhas, regiões HII e de } \\
\text { formação estelar sobrepostas }\end{array}$ & $\begin{array}{l}\text { Predominantemente estrelas azuis com } \\
\text { estrelas vermelhas espalhadas }\end{array}$ \\
\hline Halo: Estelar & Provável & Provável \\
\hline : Plasma & $\begin{array}{l}\text { Emissão de raios-x difusa, diminui com } \\
\text { a distância ao centro. Plasma térmico } \\
\text { opticamente fino com } \sim 2.10^{6} \mathrm{~K} \text { na } \\
\text { parte oeste; } \sim 10^{7} \mathrm{~K} \text { próximo a região } \\
\text { de formação estelar ativa } 30 \text { Doradus }\end{array}$ & $\begin{array}{l}\text { Emissão de raio-x difusa, plasma tér- } \\
\text { mico opticamente fino de } \sim 10^{6} \mathrm{~K} \text {; halo } \\
\text { de plasma? }\end{array}$ \\
\hline
\end{tabular}

Notas - A região central da SMC é considerada mais azul do que as regiões externas. Esse efeito é menos óbvio na LMC. A alta porcentagem de gás ainda não utilizado nas Nuvens mostra que ambas estão evolutivamente atrasadas com relação à Galáxia. Isso também é evidente pelo seu menor conteúdo metálico.

Não existem nuvens moleculares. Faltam nuvens de $\mathrm{HI}$ e $\mathrm{H}_{2}$ aquecidas pela radiação UV das estrelas quentes na SMC.

passagens perigalácticas da LMC pela MW. Rafelski e Zaritsky (2005) argumentam que a distribuição de idades de aglomerados na SMC mostra alguns picos, mas nenhum intervalo significante como na LMC (Chiosi et al., 2006). Carrera et al. (2008) investigaram a CEH da LMC e SMC a partir de relações idade-metalicidade. Há menos estudos acerca do CEH da SMC comparado a LMC, provavelmente devido a (i) sua aparência irregular, com cinemática complexa, (ii) sua distância, maior do que a da LMC, e (iii) sua profundidade na linha de visada, o que ainda gera controvérsias.

Um dos principais vínculos dos modelos de formação e evolução química de galáxias é a função distribuição de metalicidade (MDF, metallicity distribution function) das estrelas que constituem suas componentes - bojo, disco e halo. A MDF fornece informações sobre o $\mathrm{CEH}$; no halo, por exemplo, o enriquecimento em metais no Universo antigo pode fornecer detalhes sobre as primeiras gerações estelares. 
Embora no passado alguns trabalhos tenham apontado para uma ineficiente mistura química nas MC (Spite, 1989), atualmente há muitas evidências de que o meio interestelar (ISM, interstellar medium) da LMC seja relativamente bem misturado (Wang et al., 2009). Há estudos que caminham no sentido de correlacionar o ISM das Nuvens ao da MW. Razões isotópicas de carbono, nitrogênio e oxigênio demonstram que a região externa da MW não fornece uma ligação com o ISM da vizinhança solar e da LMC (Westerlund, 1997). Isto se deve provavelmente à idade das populações estelares da LMC serem elevadas quando comparadas às da Galáxia.

\subsection{Carbono em estrelas das Nuvens}

As estrelas de carbono são consideradas como pertencentes a uma população de idade intermediária e normalmente utilizadas como traçadoras de populações estelares em galáxias vizinhas à nossa. Elas são suficientemente luminosas para serem identificadas facilmente nas MC (Blanco e McCarthy, 1983, 1990; Westerlund, 1983).

O recente interesse nas fases finais da evolução de estrelas favoreceu a disponibilização de uma enorme quantidade de dados observacionais de estrelas AGB, tanto em aglomerados quanto no campo das MC. As estrelas de carbono nas MC têm sido normalmente identificadas com base em espectroscopia de baixa resolução e fotometria infravermelha, do tipo JHK e VRI. Quando possível, espectroscopia de média resolução é utilizada na confirmação do tipo espectral. Tanto fotometria quanto espectroscopia indicam que tais estrelas cobrem amplos intervalos de luminosidade, temperatura e conteúdo de carbono (Westerlund, 1991), o que é consequência de sua evolução estelar. Tamanha diversidade torna fundamental a obtenção do mais completo cenário da população de tais estrelas nas Nuvens, para que seja possível caracterizá-las adequadamente. O número de estrelas C nas MC foi estimado por Blanco e McCarthy (1983) em aproximadamente 11000 na LMC e em 2900 na SMC.

Nas últimas décadas, uma série de surveys foi feita nas MC em busca de tais estrelas ricas em carbono. Nenhum deles foi satisfatoriamente completo: não foram suficientemente profundos (Sanduleak e Philip, 1977; Westerlund et al., 1978) ou cobriram regiões restritas das nuvens (Blanco et al., 1980; Blanco e McCarthy, 1990). O trabalho de Demers et al. (1993) descreveu a busca fotométrica por estrelas vermelhas nas regiões dos halos externos 
e entre as MC. Mais da metade dessas estrelas vermelhas se revelaram como sendo estrelas de carbono. Tal resultado ressalta a participação destas na investigação da dinâmica das interações entre as componentes do Sistema de Magalhães.

\subsection{Objetivos}

Neste projeto são utilizados os dados espectroscópicos obtidos com o telescópio Magellan Clay, de 11 estrelas da SMC e outras 17 da LMC. É feito um levantamento das informações fotométricas das estrelas disponibilizadas na literatura.

Busca-se a classificação dos objetos da amostra, distinguindo as estrelas C de outros tipos espectrais, identificando assim as estrelas candidatas a CEMP. Isso permitirá esclarecer as relações entre estrelas C e estrelas CEMP, além de estabelecer a base para futura investigação da sobreabundância de carbono e da correlação desse fenômeno entre as duas populações, aparentemente distintas. A quantidade de carbono bem como a existência ou não de binaridade nas estrelas da amostra são indícios das possíveis fontes da sobreabundância do carbono.

No capítulo 2 são apresentados os dados utilizados ao longo do trabalho e detalhes dos procedimentos envolvidos em sua redução. No capítulo 3 é feita a análise dos dados reunidos. Em seguida, no capítulo 4, são apresentadas as principais conclusões e perspectivas para o futuro do projeto. 


\section{Capítulo 2}

\section{Base de dados}

\subsection{A amostra de candidatas a estrelas CEMP das Nuvens de Magalhães}

A amostra de estrelas candidatas a CEMP, que constitui a base de dados estudada, é composta por 28 objetos das Nuvens de Magalhães, sendo 11 da SMC e as outras 17 da LMC.

Os objetos foram selecionadas por Marcos Diaz, Ronald Mennickent e Deonisio Cieslinski, a partir das curvas de luz do catálogo OGLE. O critério de seleção da amostra, que visava a identificação de estrelas candidatas a variáveis irregulares, levou em conta a variação da amplitude de brilho e a presença de peculiaridades nas curvas de luz (tais como irregularidade no padrão de comportamento da curva, modulação regular porém com aumento ou declínio acentuado e de outras características não vistas em Miras). A aplicação de tais critérios resultou em uma amostra com 92 estrelas presentes no catálogo OGLE, das quais 28 já foram observadas espectroscopicamente no telescópio Magellan Clay. Após tal missão observacional espectroscópica, os dados foram reduzidos e o grupo verificou que os critérios escolhidos para o processo de seleção resultaram em uma amostra composta por uma grande quantidade de estrelas com espectros contendo fortes bandas de absorção de moléculas de carbono.

É verossímil as 28 estrelas serem estrelas C dada a fácil identificação visual das bandas moleculares de carbono nos espectros, apresentados nas Figuras 2.1 até 2.5. É essa característica que define a amostra como composta por candidatas a estrelas CEMP. A Tabela 2.1 apresenta informações gerais sobre as estrelas da amostra deste trabalho. O identificador OGLE está na coluna 1 da Tabela 2.1, as colunas 2 e 3 apresentam as coordenadas equatoriais e galácticas, a nuvem de origem encontra-se na 4, o número de espectros obtidos 
na coluna 5 e os tempos de exposição na coluna 6. Nas Figuras 2.6 e 2.7 são apresentados dois gráficos que fornecem a localização dos objetos no espaço de coordenadas galácticas e são assinalados os centroides das duas Nuvens, fornecidos por de Vaucouleurs et al. (1976) através do NED ${ }^{1}$.

Foi feita busca por informações dos objetos no Simbad $^{2}$. O único sem resposta para a consulta por identificadores (OGLE e MACHO) foi OGLE J004456.46-731224.2; ainda assim, houve resposta na busca por suas coordenadas. Logo, todos os objetos já foram observados por algum survey embora nenhum deles tenha sido citado como objeto particular de um estudo.

\subsection{Espectroscopia}

A espectroscopia em média resolução da amostra $(R \sim 1500, \Delta \lambda \sim 4,5 \AA)$ foi feita no Telescópio Magellan Clay de 6,5m com o espectrógrafo Boller \& Chivens (com o CCD Marconi\#1 2048x515) da Carnegie Institution of Washington nos dias 30 de outubro e 1 de novembro de 2004. Os espectros possuem razão sinal/ruído (S/R) de, em média, 45/1 na região de $6000 \AA$ e estão calibrados em fluxo em um intervalo de comprimento de onda entre 3850 e $7000 \AA$. Para cada alvo foram obtidos de 2 a 6 espectros com exposições de 30, 60, 100, 200 ou 300 segundos; o tempo de exposição foi sempre o mesmo para cada objeto.

Com o intuito de se reduzir as incertezas dos fluxos calibrados, os objetos tiveram seus vários espectros combinados em versões únicas. Para isso, foram utilizadas as rotinas scombine e scopy do pacote de ferramentas de análise de dados astronômicos IRAF ${ }^{3}$. Os espectros foram combinados via média simples, uma vez que o tempo de exposição das observações não varia para um mesmo objeto. Os espectros resultantes possuem S/R 45/1 na região de $6000 \AA$ A. As Figuras 2.1 até 2.5 mostram os espectros dos 28 objetos da amostra

\footnotetext{
${ }^{1}$ Esta pesquisa recorreu ao NASA/IPAC Extragalactic Database (NED), operado pelo Jet Propulsion Laboratory, California Institute of Technology, sob contrato com National Aeronautics and Space Administration.

${ }^{2}$ A pesquisa fez uso da base de dados Simbad, operados pelo CDS, Strasbourg, França (http://simbad.ustrasbg.fr/simbad/)

${ }^{3}$ Image Reduction and Analysis Facility é distribuído pelo National Optical Astronomical Observatories (NOAO), o qual é operado pela Association of Universities for Research in Astronomy (AURA), sob contrato do National Science Foundation (NSF).
} 
Tabela 2.1 - Log das observações dos 28 objetos.

\begin{tabular}{|c|c|c|c|c|c|}
\hline Nome & $\alpha, \delta(\mathrm{J} 2000)$ & $\mathrm{l}, \mathrm{b}$ & Local & $\mathrm{N}$ & Exposição \\
\hline OGLE & hh:mm:ss.ss, dd:mm:ss.s & ddd:mm:ss.s, dd:mm:ss.s & & & $\mathrm{s}$ \\
\hline (1) & $(2)$ & $(3)$ & (4) & $(5)$ & (6) \\
\hline J003722.15-732222.8 & $00: 37: 22.15,-73: 22: 25.0$ & $304: 19: 25.2,-43: 43: 02.0$ & $\mathrm{SMC}$ & 3 & 200 \\
\hline J004032.22-732048.3 & $00: 40: 32.94,-73: 20: 49.1$ & 304:00:47.0, -43:45:31.4 & $\mathrm{SMC}$ & 3 & 100 \\
\hline J004216.08-725731.9 & $00: 42: 15.30,-72: 57: 29.6$ & $303: 52: 06.3,-44: 09: 09.1$ & $\mathrm{SMC}$ & 3 & 30 \\
\hline J004456.46-731224.2 & $00: 44: 56.48,-73: 12: 25.7$ & $303: 35: 00.8,-43: 54: 48.2$ & $\mathrm{SMC}$ & 3 & 200 \\
\hline J005058.90-730006.6 & 00:50:58.47, -73:00:03.4 & $302: 58: 42.7,-44: 07: 34.3$ & $\mathrm{SMC}$ & 3 & 60 \\
\hline J005106.53-730502.8 & 00:51:06.55, -73:05:09.2 & $302: 57: 55.6,-44: 02: 38.3$ & $\mathrm{SMC}$ & 3 & 200 \\
\hline J005401.40-731029.8 & 00:54:02.33, -73:10:33.3 & $302: 40: 19.5,-43: 57: 07.7$ & $\mathrm{SMC}$ & 2 & 200 \\
\hline J005516.51-725733.4 & $00: 55: 17.39,-72: 57: 37.2$ & $302: 32: 24.2,-44: 09: 58.7$ & $\mathrm{SMC}$ & 3 & 200 \\
\hline J005835.16-725935.4 & $00: 58: 35.10,-72: 59: 31.9$ & $302: 12: 13.4,-44: 07: 29.3$ & $\mathrm{SMC}$ & 3 & 200 \\
\hline J005929.08-723926.7 & $00: 59: 29.12,-72: 39: 24.7$ & $302: 05: 31.4,-44: 27: 28.3$ & $\mathrm{SMC}$ & 3 & 200 \\
\hline J010846.90-724019.4 & $01: 08: 47.33,-72: 40: 16.6$ & $301: 07: 32.2,-44: 23: 44.2$ & $\mathrm{SMC}$ & 3 & 300 \\
\hline J050515.90-684446.6 & $05: 05: 16.54,-68: 44: 44.3$ & $279: 42: 11.6,-34: 41: 26.7$ & $\mathrm{LMC}$ & 3 & 100 \\
\hline J051139.99-684816.8 & $05: 11: 40.71,-68: 48: 15.3$ & $279: 36: 22.8,-34: 06: 48.9$ & $\mathrm{LMC}$ & 6 & 100 \\
\hline J051544.81-694457.1 & $05: 15: 45.92,-69: 44: 57.5$ & $280: 37: 11.5,-33: 33: 12.7$ & $\mathrm{LMC}$ & 3 & 200 \\
\hline J051609.74-693517.9 & $05: 16: 10.26,-69: 35: 19.1$ & $280: 25: 18.7,-33: 33: 10.5$ & $\mathrm{LMC}$ & 5 & 200 \\
\hline J051810.88-692626.5 & $05: 18: 10.48,-69: 26: 20.7$ & $280: 12: 16.4,-33: 24: 38.7$ & $\mathrm{LMC}$ & 3 & 300 \\
\hline J051954.02-692802.8 & 05:19:53.86, -69:27:55.8 & $280: 12: 01.5,-33: 15: 26.4$ & LMC & 3 & 200 \\
\hline J051958.26-691416.7 & $05: 19: 58.31,-69: 14: 09.4$ & $279: 55: 44.9,-33: 17: 46.9$ & $\mathrm{LMC}$ & 3 & 300 \\
\hline J052047.00-695046.2 & $05: 20: 47.29,-69: 50: 38.8$ & $280: 37: 30.3,-33: 06: 28.5$ & $\mathrm{LMC}$ & 3 & 300 \\
\hline J052154.24-705657.0 & $05: 21: 54.59,-70: 56: 49.0$ & $281: 53: 27.0,-32: 47: 41.2$ & LMC & 3 & 300 \\
\hline J052555.11-694354.1 & $05: 25: 55.80,-69: 43: 52.9$ & $280: 23: 36.5,-32: 41: 35.8$ & $\mathrm{LMC}$ & 3 & 200 \\
\hline J053424.83-694400.1 & $05: 34: 25.40,-69: 43: 51.7$ & $280: 15: 34.8,-31: 57: 60.0$ & $\mathrm{LMC}$ & 3 & 300 \\
\hline J053608.58-702220.5 & 05:36:09.28, $-70: 22: 19.6$ & $280: 58: 50.7,-31: 43: 59.6$ & $\mathrm{LMC}$ & 6 & 100 \\
\hline J053900.00-695225.1 & 05:39:00.63, -69:52:24.5 & $280: 21: 47.8,-31: 33: 21.8$ & $\mathrm{LMC}$ & 3 & 200 \\
\hline J053910.32-695916.7 & $05: 39: 11.05,-69: 59: 16.2$ & $280: 29: 39.1,-31: 31: 39.3$ & $\mathrm{LMC}$ & 3 & 200 \\
\hline J054223.01-701810.3 & 05:42:23.71, -70:18:01.6 & $280: 49: 23.2,-31: 13: 09.3$ & $\mathrm{LMC}$ & 2 & 200 \\
\hline J054433.79-703949.1 & $05: 44: 34.50,-70: 39: 48.2$ & 281:13:09.5, -31:00:01.1 & $\mathrm{LMC}$ & 3 & 200 \\
\hline J054658.32-702505.6 & $05: 46: 58.86,-70: 25: 04.9$ & $280: 54: 39.5,-30: 49: 29.1$ & $\mathrm{LMC}$ & 3 & 200 \\
\hline
\end{tabular}


de candidatas a estrelas CEMP. Cada janela contém o respectivo identificador OGLE.

\subsection{Projeto OGLE e curvas de luz}

O Projeto OGLE (Optical Gravitational Lensing Experiment; Udalski, 2003) tem como objetivo procurar fenômenos de microlentes que manifestam interações da matéria escura com a bariônica. Tal ideia foi proposta por Paczynski (1986, 1991). As MC e o bojo Galáctico são os sítios mais apropriados para a condução do projeto devido ao grande número de estrelas que podem sofrer efeitos de lente causados por objetos no halo e no bojo da Galáxia.

A primeira fase do projeto (OGLE-I) teve início em 1992 e seguiu até 1995. Nesse período, foi utilizado o telescópio Swope de 1m, localizado no Las Campanas Observatory, no Chile, operado pelo Carnegie Institution of Washington com uma câmera CCD Ford/Loral 2kx2k, sendo as observações limitadas ao bojo Galáctico. Em 1996, com a conclusão do telescópio Warsaw de 1,3m, localizado no mesmo sítio observacional e operado pelo Carnegie Institution of Washington, Princeton University e Warsaw University Observatory, a segunda fase (OGLE-II) pôde ser iniciada e, desde então, as Nuvens de Magalhães vêm sendo observadas, utilizando um CCD SITE $2 k x 2 k$. A terceira fase (OGLE-III) teve início em 2001. Essa versão do projeto utiliza a fotometria DIA (difference image analysis) no lugar do ajuste de perfil PSF (point source fitting). Tal metodologia aumentou substancialmente a taxa de detecção de eventos de microlentes estelares pelo projeto OGLE.

As curvas de luz na magnitude I do sistema fotométrico padrão Johnson e Morgan (1953) dos 28 objetos que constituem a amostra foram obtidas durante o OGLE-III. A variabilidade de luz pode ser um indicativo de que os objetos fazem parte de sistemas binários. Havendo binaridade, a hipótese de que tais sistemas sustentam processos de enriquecimento em carbono em estrelas VMP pode ser melhor analisada. As Figuras 2.1 a 2.5 apresentam também as curvas de luz dos objetos da amostra.

\subsection{Fotometria $\mathrm{IJHK}_{s}$}

A estimativa dos parâmetros atmosféricos das estrelas candidatas a CEMP, como por exemplo temperatura efetiva e gravidade superficial, exige informações fotométricas nas 


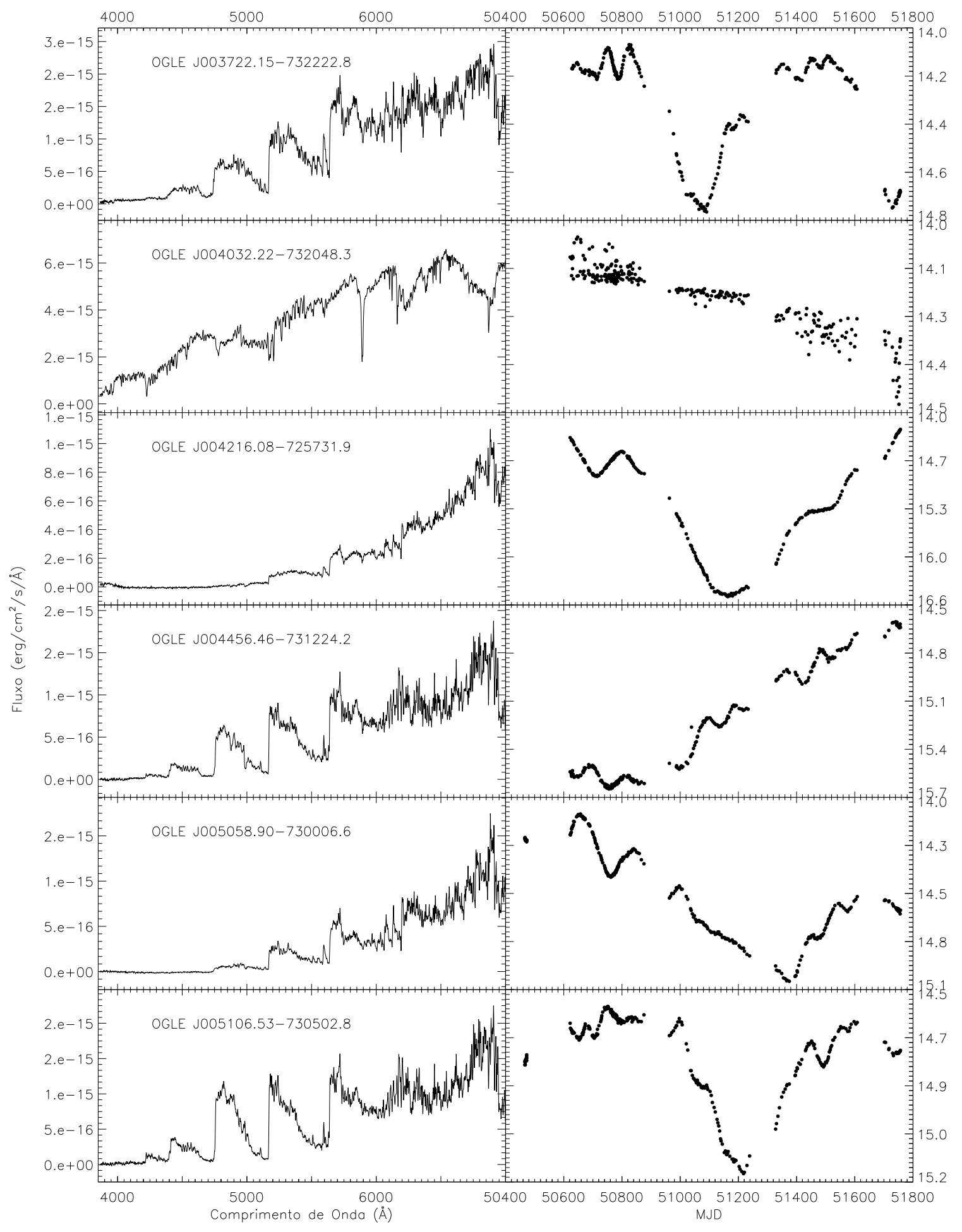

Figura 2.1: Espectros e curvas de luz (magnitude I) das estrelas da SMC (1). 


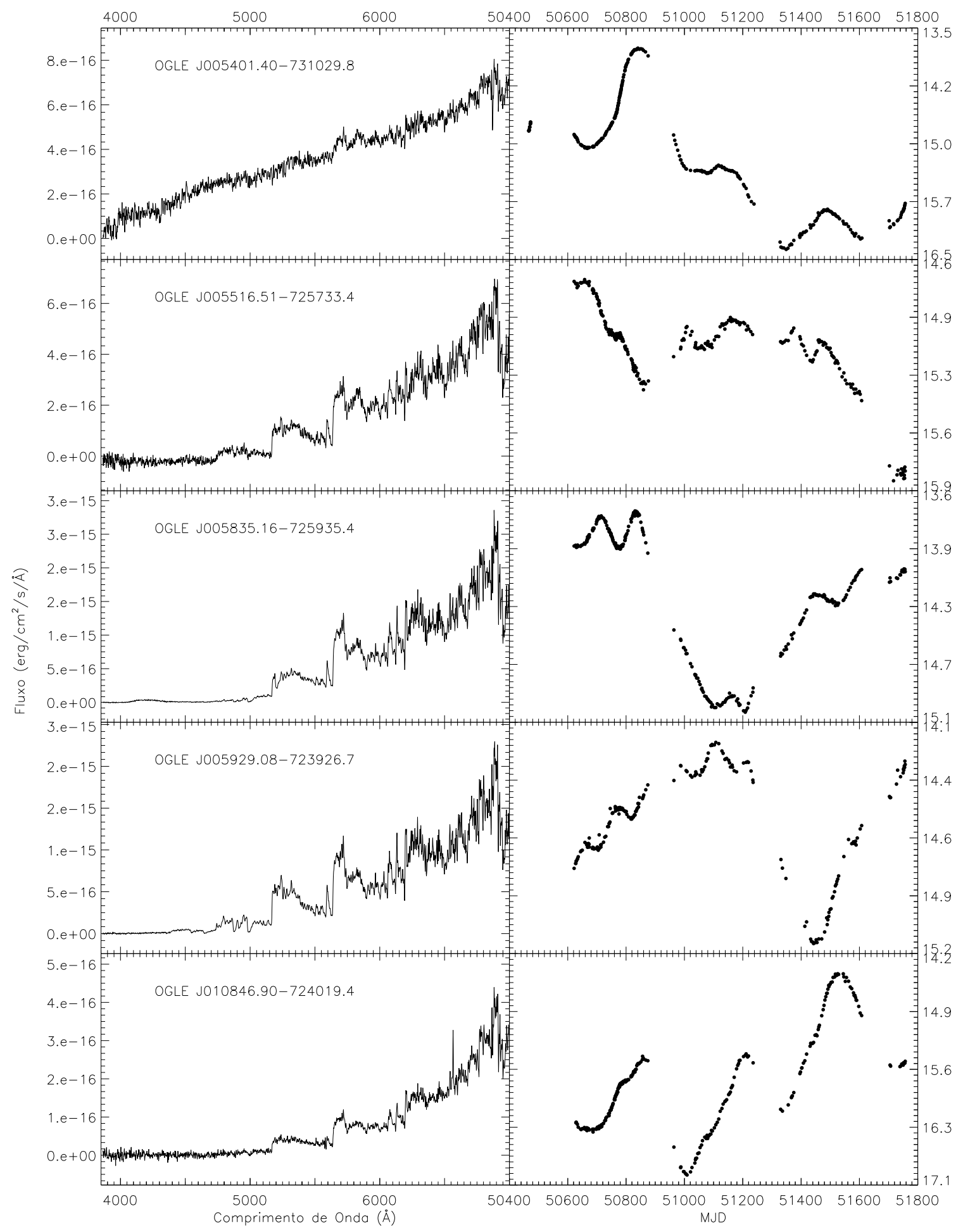

Figura 2.2: Espectros e curvas de luz (magnitude I) das estrelas da SMC (2). 


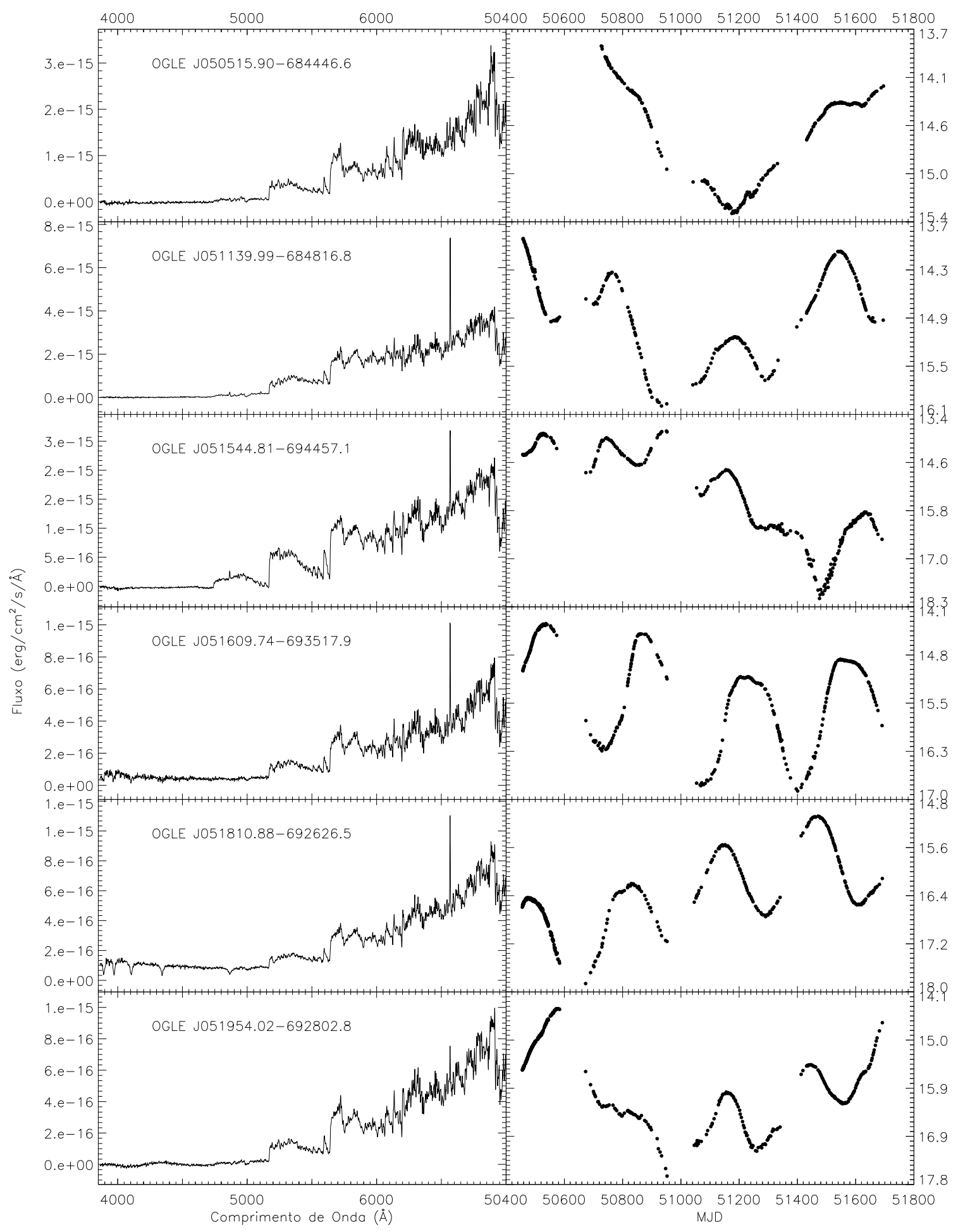

Figura 2.3: Espectros e curvas de luz (magnitude I) das estrelas da LMC (1). 


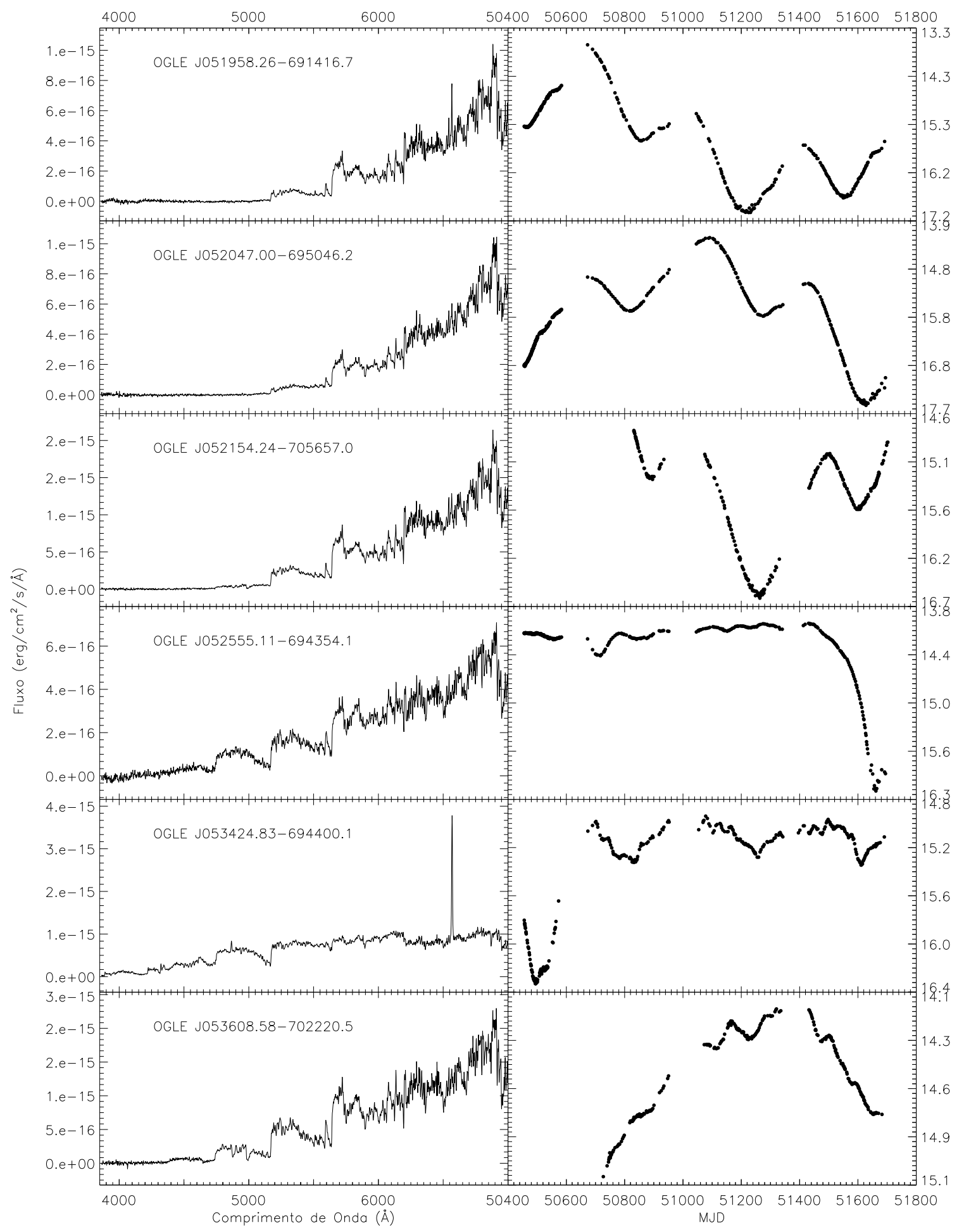

Figura 2.4: Espectros e curvas de luz (magnitude I) das estrelas da LMC (2). 


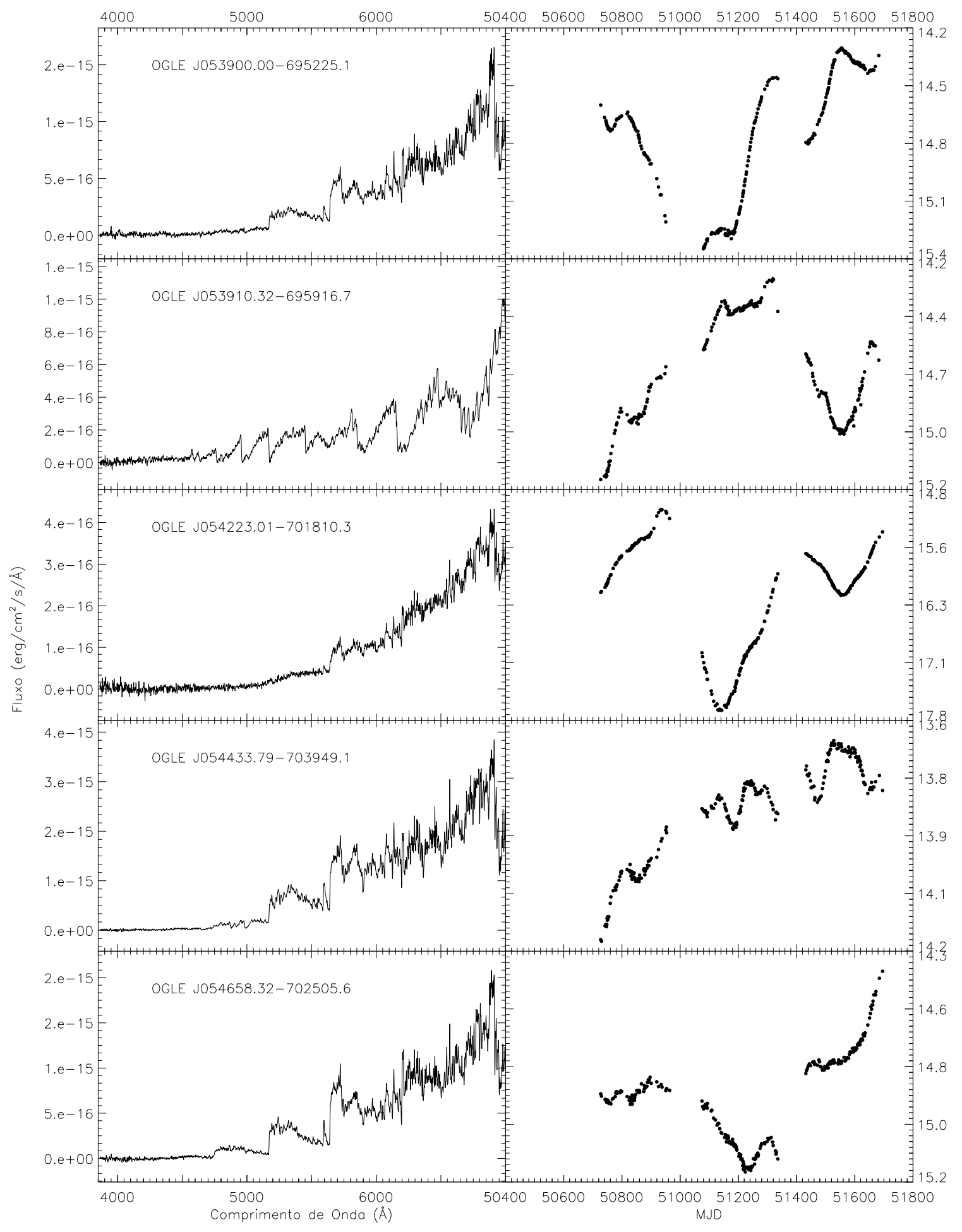

Figura 2.5: Espectros e curvas de luz (magnitude I) das estrelas da LMC (3). 


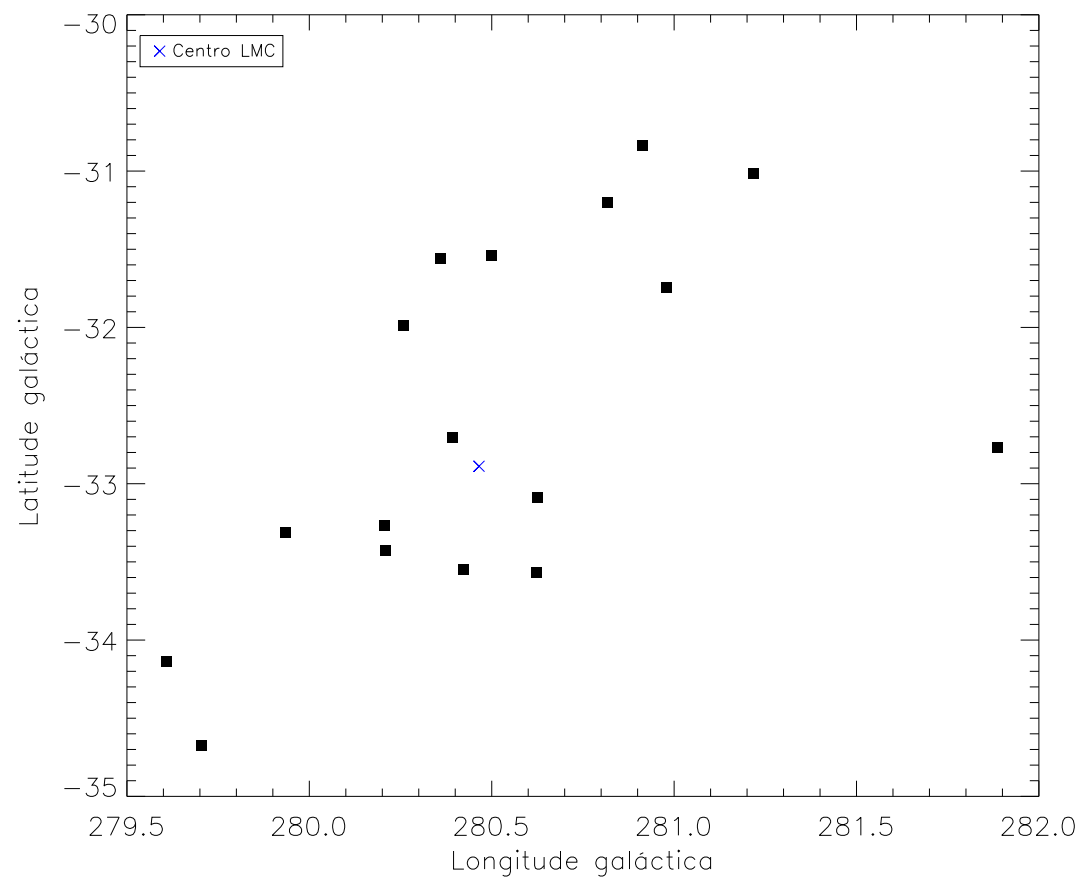

Figura 2.6: Distribuição dos objetos da amostra na LMC no espaço de coordenadas galácticas. O "x" representa o centroide da Nuvem.

regiões do vermelho e infravermelho próximo do espectro. Os dados fotométricos da amostra são apresentados nas Tabelas 2.2 e 2.3. Por serem objetos variáveis, é de se esperar que tal variabilidade aumente o erro na determinação dos valores de magnitudes tanto na banda I quanto nas outras bandas e, portanto, implique em incertezas nos parâmetros derivados a partir da fotometria.

As magnitudes I médias (coluna 4 da Tabela 2.2) foram fornecidas pelo OGLE-III, com uma média das incertezas na magnitude I da ordem de 0,01. No entanto, a maior fonte de erro nessa magnitude é a variabilidade de brilho dos objetos: o desvio padrão das magnitudes varia de 0,1 a 1,2 de objeto para objeto.

A fotometria $\mathrm{JHK}_{s}$ foi obtida a partir do catálogo 2MASS ${ }^{4}$ (Skrutskie et al., 2006). Sua aquisição, baseada em um sistema fotométrico próprio, é descrita em detalhes no trabalho de Cohen et al. (2003). As magnitudes, apresentadas nas colunas 5, 6 e 7 da Tabela

\footnotetext{
${ }^{4} \mathrm{O}$ trabalho utilizou informações produzidas pelo Two Micro All Sky Survey (2MASS), o qual faz parte do projeto da University of Massachusetts e do Infrared Processing and Analysis Center/California Institute of Technology, financiado por National Aeronautics and Space Administration e por National Science Foundation.
} 


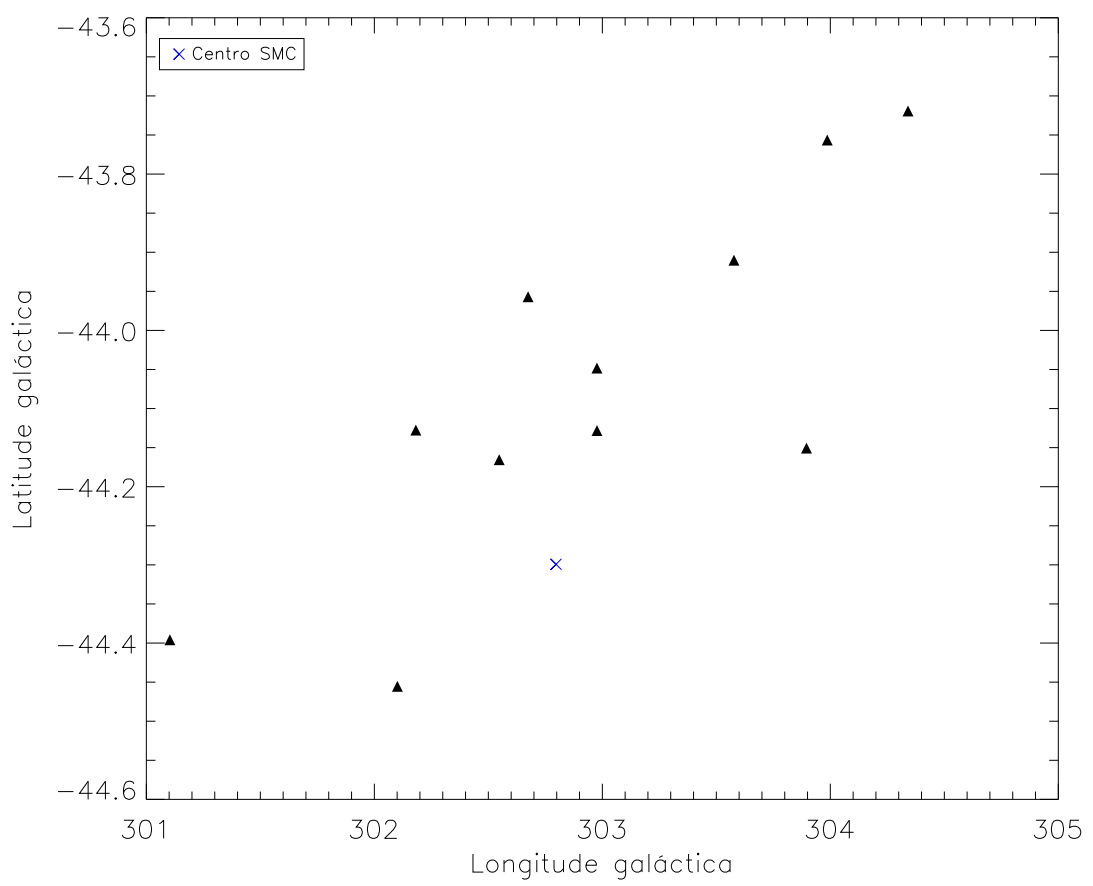

Figura 2.7: Distribuição dos objetos da amostra na SMC no espaço de coordenadas galácticas. O "x" representa o centroide da Nuvem.

2.2, foram acessadas via catálogo $\operatorname{VizieR}^{5}$ (Ochsenbein et al., 2000). Foram estabelecidos critérios de seleção de informações a fim de se assegurar a obtenção da fotometria $\mathrm{JHK}_{s}$ correta para toda a amostra. As buscas consideraram (i) um raio de 10" em torno das coordenadas contidas nos cabeçalhos dos espectros, (ii) os identificadores OGLE e (iii) os identificadores MACHO. Para as coordenadas com retorno múltiplo, foi escolhida a fotometria que teve um único retorno na busca por identificadores, obviamente idêntico a um dos resultados retornados pela busca por coordenadas. Para quatro estrelas, não houve nenhum retorno na busca por identificadores; neste caso, foram escolhidas as magnitudes com menor raio em torno das respectivas coordenadas da busca. A média das incertezas das magnitudes $\mathrm{J}, \mathrm{H}$ e $\mathrm{K}_{s}$, fornecidas pelo catálogo 2MASS, é 0,03 . As cores $\mathrm{J}-\mathrm{H}, \mathrm{H}-\mathrm{K}_{s}$ e $\mathrm{J}-\mathrm{K}_{s}$ são apresentadas nas colunas 13, 14 e 15 da Tabela 2.2.

A magnitude $\mathrm{K}_{s}$ adotada pelo sistema fotométrico do 2MASS é diferente da magnitude K do sistema de Bessell e Brett (1988) devido a ligeiras diferenças entre os filtros utilizados.

\footnotetext{
${ }^{5}$ A pesquisa fez uso da ferramenta de acesso a catálogos VizieR, operados pelo CDS, Strasbourg, França (http://webviz.u-strasbg.fr/viz-bin/VizieR/).
} 
As relações entre as magnitudes $\mathrm{K}$ e $\mathrm{K}_{s}$ e as cores $\mathrm{J}-\mathrm{K}$ e $\mathrm{J}-\mathrm{K}_{s}$, extraídas do Complemento Explanatório do 2MASS All Sky Data Release ${ }^{6}$, são dadas pelas Equações

$$
\begin{aligned}
& K_{s}=K+(-0,039 \pm 0,007)+(0,001 \pm 0,005)(J-K) \mathrm{e} \\
& \left(J-K_{s}\right)=(0,983 \pm 0,008)(J-K)+(-0,018 \pm 0,007) .
\end{aligned}
$$

As magnitudes $\mathrm{K}_{s}$ dos objetos da amostra são, em média, 0,026 menores que as magnitudes $\mathrm{K}$, apresentadas na coluna 8 da Tabela 2.2 , enquanto $\mathrm{J}-\mathrm{K}_{s}$ (2MASS) é em média 0,05 menor que J-K no sistema de Bessel e Brett.

\subsection{Avermelhamento}

Quando se usa ou se interpreta cores de estrelas, deve-se sempre ter em mente que as cores observadas são afetadas pela extinção interestelar, causada por gás e poeira que espalham e absorvem a luz proveniente das estrelas. A extinção varia de acordo com cada banda passante, ou seja, não é uniforme para todo o espectro eletromagnético. Assim, a curva de extinção seletiva costuma ser parametrizada em termos da extinção na banda $\mathrm{V}\left(\mathrm{R}_{V}\right)$ e de uma medida da extinção relativa entre as bandas $\mathrm{B}$ e $\mathrm{V}$, de acordo com a Equação 2.2,

$$
R_{V}=\frac{A(V)}{A(V)-A(B)} \equiv \frac{A(V)}{E(B-V)},
$$

onde $\mathrm{A}(\mathrm{V})$ (ou $\mathrm{A}_{V}$ ) é o avermelhamento na banda $\mathrm{V}$ e $\mathrm{E}(\mathrm{B}-\mathrm{V})$ é o avermelhamento do índice de cor B-V, também conhecido como excesso de cor. Diz-se avermelhamento porque a região azul do espectro é mais afetada pela extinção do que a vermelha, fazendo com que a luz proveniente das estrelas se torne avermelhada. Evidências observacionais mostram que $\mathrm{R}_{V}$ varia de 2,6 a 5,5 (Clayton e Cardelli, 1988).

A estimativa de avermelhamento pode ser feita de diversas formas. Uma das técnicas utiliza mapas de distribuição de poeira galáctica como os de Burstein e Heiles (1978, 1982) na correção do avermelhamento. Esse mapeamento, construído a partir de surveys de colunas de densidade de hidrogênio neutro (Heiles e Jenkins, 1976), vem sendo uma importante referência para toda comunidade astrofísica devido a sua maior precisão em relação às estimativas anteriores. Schlegel et al. (1998, daqui em diante, SFD98) propuseram novos mapas que são tão bons ou melhores que os de Burstein e Heiles (1978). Esses mapas de

\footnotetext{
${ }^{6}$ http://www.ipac.caltech.edu/2mass/releases/allsky/doc/explsup.html
} 
Tabela 2.2 - Informações sobre a amostra de estrelas das MC. São apresentados: identificadores OGLE e MACHO, magnitudes V, I média, J, H, K ${ }_{s}$ e K, extinção $\mathrm{A}_{V}$ e $\mathrm{A}_{K}$, avermelhamento $\mathrm{E}\left(\mathrm{J}-\mathrm{K}_{s}\right)$ e $\mathrm{E}\left(\mathrm{V}-\mathrm{K}_{s}\right)$ e índices de cor $\mathrm{J}-\mathrm{H}, \mathrm{H}-\mathrm{K}_{s}, \mathrm{~J}-\mathrm{K}_{s},\left(\mathrm{~J}-\mathrm{K}_{s}\right)_{0},(\mathrm{~J}-\mathrm{K})_{0}$ e $(\mathrm{V}-\mathrm{K})_{0}$.

\begin{tabular}{|c|c|c|c|c|c|c|c|c|c|c|c|c|c|c|c|c|c|}
\hline $\begin{array}{l}\text { OGLE } \\
(1)\end{array}$ & $\begin{array}{c}\text { MACHO } \\
\text { (2) }\end{array}$ & $\begin{array}{l}\text { V } \\
(3)\end{array}$ & $\begin{array}{c}<I> \\
(4)\end{array}$ & $\begin{array}{l}\mathrm{J} \\
(5)\end{array}$ & $\begin{array}{l}\mathrm{H} \\
(6)\end{array}$ & $\begin{array}{l}\mathrm{K}_{s} \\
(7)\end{array}$ & $\begin{array}{l}\mathrm{K} \\
(8)\end{array}$ & $\begin{array}{l}\mathrm{A}_{V} \\
(9)\end{array}$ & $\begin{array}{l}\mathrm{A}_{K} \\
(10)\end{array}$ & $\begin{array}{c}\mathrm{E}\left(\mathrm{J}-\mathrm{K}_{s}\right) \\
(11)\end{array}$ & $\begin{array}{c}\mathrm{E}\left(\mathrm{V}-\mathrm{K}_{s}\right) \\
(12)\end{array}$ & $\begin{array}{l}\mathrm{J}-\mathrm{H} \\
(13)\end{array}$ & $\begin{array}{c}\mathrm{H}-\mathrm{K}_{s} \\
(14)\end{array}$ & $\begin{array}{c}\mathrm{J}-\mathrm{K}_{s} \\
(15)\end{array}$ & $\begin{array}{c}\left(\mathrm{J}-\mathrm{K}_{s}\right)_{0} \\
(16)\end{array}$ & $\begin{array}{c}(\mathrm{J}-\mathrm{K})_{0} \\
(17)\end{array}$ & $\begin{array}{c}\left(\mathrm{V}-\mathrm{K}_{s}\right)_{0} \\
(18)^{2}\end{array}$ \\
\hline J003722.15-732222.8 & 213.15162 .5 & $16,69^{\star}$ & 14,3 & 13,28 & 12,23 & 11,76 & 11,79 & 0,16 & 0,02 & 0,03 & 0,14 & 1,05 & 0,47 & 1,52 & 1,49 & 1,54 & 4,79 \\
\hline J004032.22-732048.3 & 213.15391 .10 & 14,68 & 14,2 & 11,73 & 11,17 & 10,89 & 10,92 & 0,28 & 0,03 & 0,05 & 0,25 & 0,56 & 0,28 & 0,84 & 0,78 & 0,82 & 4,87 \\
\hline J004216.08-725731.9 & 213.15453 .10 & $17,41^{\star}$ & 15,1 & 12,36 & 11,12 & 10,35 & 10,38 & 0,49 & 0,05 & 0,09 & 0,44 & 1,24 & 0,77 & 2,01 & 1,92 & 1,98 & 6,62 \\
\hline J004456.46-731224.2 & 212.15621 .153 & 16,86 & 15,3 & 13,24 & 11,97 & 11,29 & 11,32 & 1,54 & 0,17 & 0,28 & 1,38 & 1,27 & 0,68 & 1,95 & 1,67 & 1,71 & 4,21 \\
\hline J005058.90-730006.6 & 212.16023 .42 & $17,11^{\star}$ & 14,5 & 12,78 & 11,65 & 10,98 & 11,01 & 1,11 & 0,12 & 0,20 & 0,98 & 1,13 & 0,67 & 1,80 & 1,60 & 1,65 & 5,15 \\
\hline J005106.53-730502.8 & 212.16021 .35 & $16,80^{\star}$ & 14,7 & 13,06 & 11,98 & 11,45 & 11,48 & 1,05 & 0,12 & 0,19 & 0,94 & 1,08 & 0,53 & 1,61 & 1,42 & 1,47 & 4,41 \\
\hline J005401.40-731029.8 & 211.16191 .60 & 17,26 & 15,1 & 13,63 & 12,37 & 11,34 & 11,36 & 0,92 & 0,10 & 0,17 & 0,82 & 1,26 & 1,03 & 2,29 & 2,12 & 2,19 & 4,60 \\
\hline J005516.51-725733.4 & 211.16308 .19 & $16,94^{\star}$ & 15,1 & 13,26 & 12,10 & 11,30 & 11,33 & 0,58 & 0,06 & 0,10 & 0,52 & 1,16 & 0,80 & 1,96 & 1,86 & 1,91 & 5,13 \\
\hline J005835.16-725935.4 & 211.16479 .2 & $17,02^{\star}$ & 14,2 & 12,98 & 11,86 & 11,02 & 11,05 & 0,48 & 0,05 & 0,09 & 0,42 & 1,12 & 0,84 & 1,96 & 1,88 & 1,93 & 5,57 \\
\hline J005929.08-723926.7 & 207.16541 .11 & $17,60^{\star}$ & 14,6 & 12,76 & 11,70 & 11,14 & 11,17 & 0,67 & 0,07 & 0,12 & 0,59 & 1,06 & 0,56 & 1,62 & 1,49 & 1,55 & 5,86 \\
\hline J010846.90-724019.4 & 206.17168 .79 & 19,58 & 15,8 & 14,57 & 12,90 & 11,62 & 11,64 & 0,34 & 0,04 & 0,06 & 0,30 & 1,67 & 1,28 & 2,95 & 2,89 & 2,96 & 5,84 \\
\hline J050515.90-684446.6 & 1.4056 .1146 & 17,21 & 14,6 & 12,98 & 11,56 & 10,62 & 10,65 & 0,61 & 0,07 & 0,11 & 0,54 & 1,42 & 0,94 & 2,36 & 2,25 & 2,31 & 6,18 \\
\hline J051139.99-684816.8 & 79.5023 .40 & 16,27 & 14,8 & 13,44 & 12,18 & 11,36 & 11,39 & 1,55 & 0,20 & 0,28 & 1,38 & 1,26 & 0,82 & 2,08 & 1,80 & 1,80 & 4,28 \\
\hline J051544.81-694457.1 & 78.5735 .2240 & 16,95 & 15,3 & 13,48 & 12,43 & 11,75 & 11,78 & 1,13 & 0,12 & 0,20 & 1,01 & 1,05 & 0,68 & 1,73 & 1,52 & 1,59 & 4,68 \\
\hline J051609.74-693517.9 & 78.5737 .19 & 18,28 & 15,3 & 13,37 & 11,85 & 10,70 & 10,73 & 0,89 & 0,10 & 0,16 & 0,80 & 1,52 & 1,15 & 2,67 & 2,51 & 2,57 & 5,60 \\
\hline J051810.88-692626.5 & 78.6102 .490 & 18,03 & 16,4 & 13,51 & 11,99 & 10,91 & 10,94 & 3,25 & 0,34 & 0,58 & 2,90 & 1,52 & 1,08 & 2,60 & 2,01 & 2,10 & 3,09 \\
\hline J051954.02-692802.8 & 78.6465 .85 & 18,31 & 15,6 & 14,17 & 12,74 & 11,67 & 11,70 & 2,39 & 0,28 & 0,43 & 2,13 & 1,43 & 1,07 & 2,50 & 2,07 & 2,10 & 3,78 \\
\hline J051958.26-691416.7 & 80.6468 .77 & 18,88 & 15,5 & 14,28 & 12,66 & 11,36 & 11,39 & 2,75 & 0,35 & 0,49 & 2,45 & 1,62 & 1,30 & 2,92 & 2,43 & 2,41 & 3,83 \\
\hline J052047.00-695046.2 & 78.6580 .62 & 18,91 & 16,0 & 12,70 & 11,28 & 10,24 & 10,27 & 1,85 & 0,21 & 0,33 & 1,65 & 1,42 & 1,04 & 2,46 & 2,12 & 2,17 & 5,76 \\
\hline J052154.24-705657.0 & 13.6685 .29 & 17,56 & 15,6 & 13,86 & 12,41 & 11,41 & 11,44 & 0,37 & 0,04 & 0,07 & 0,32 & 1,45 & 1,00 & 2,45 & 2,39 & 2,45 & 5,56 \\
\hline J052555.11-694354.1 & 77.7429 .64 & 18,07 & 14,3 & 12,75 & 11,58 & 10,97 & 10,99 & 1,19 & 0,15 & 0,21 & 1,06 & 1,17 & 0,61 & 1,78 & 1,56 & 1,59 & 5,26 \\
\hline J053424.83-694400.1 & 81.8760 .67 & 16,48 & 15,4 & 13,65 & 12,82 & 12,59 & 12,62 & 3,02 & 0,30 & 0,54 & 2,69 & 0,83 & 0,23 & 1,06 & 0,52 & 0,60 & 1,35 \\
\hline J053608.58-702220.5 & 11.8992 .23 & 16,92 & 14,5 & 12,78 & 11,59 & 10,89 & 10,92 & 0,93 & 0,10 & 0,17 & 0,83 & 1,19 & 0,70 & 1,89 & 1,73 & 1,78 & 5,29 \\
\hline J053900.00-695225.1 & 81.9484 .20 & 17,84 & 14,7 & 12,65 & 11,25 & 10,38 & 10,41 & 5,42 & 0,61 & 0,97 & 4,83 & 1,40 & 0,87 & 2,27 & 1,30 & 1,32 & 2,09 \\
\hline J053910.32-695916.7 & 81.9482 .51 & 18,25 & 14,7 & 12,58 & 11,53 & 11,14 & 11,17 & 3,70 & 0,40 & 0,66 & 3,30 & 1,05 & 0,39 & 1,44 & 0,77 & 0,82 & 2,79 \\
\hline J054223.01-701810.3 & 12.10082 .100 & 19,51 & 16,2 & 13,28 & 11,73 & 10,64 & 10,67 & 1,57 & 0,16 & 0,28 & 1,40 & 1,55 & 1,09 & 2,64 & 2,35 & 2,44 & 5,71 \\
\hline J054433.79-703949.1 & 12.10440 .10 & 16,50 & 13,8 & 12,10 & 10,98 & 10,37 & 10,39 & 1,39 & 0,15 & 0,25 & 1,24 & 1,12 & 0,61 & 1,73 & 1,49 & 1,53 & 5,41 \\
\hline J054658.32-702505.6 & 12.10807 .15 & 17,37 & 14,9 & 12,71 & 11,42 & 10,59 & 10,62 & 1,35 & 0,15 & 0,24 & 1,20 & 1,29 & 0,83 & 2,12 & 1,88 & 1,93 & 5,43 \\
\hline
\end{tabular}

*indica magnitude $\mathrm{V}$ adotada como padrão. 
Tabela 2.3 - Magnitudes absolutas $\mathrm{M}_{V}$ e $\mathrm{M}_{K}$, magnitudes bolométricas $\mathrm{M}_{b o l}^{A}$ e $\mathrm{M}_{b o l}^{W}$, velocidades radiais heliocêntricas e tipos espectrais (ST) dos objetos da amostra.

\begin{tabular}{|c|c|c|c|c|c|c|}
\hline $\begin{array}{c}\text { Nome } \\
\text { OGLE } \\
(1)\end{array}$ & $\mathrm{M}_{V}$ & $\mathrm{M}_{K}$ & $\mathrm{M}_{b o l}^{A}$ & $\mathrm{M}_{b o l}^{W}$ & $\begin{array}{c}\mathrm{v}_{\text {rad }} \\
\mathrm{km} / \mathrm{s} \\
(6)\end{array}$ & $\mathrm{ST}$ \\
\hline J003722.15-732222.8 & $-2,30$ & $-7,14$ & $-4,34$ & $-4,10$ & 155,8 & $\mathrm{C}-\mathrm{N} 5$ \\
\hline J004032.22-732048.3 & $-3,09$ & $-8,02$ & $-5,15$ & $-5,75$ & $6,4^{\dagger}$ & $\mathrm{dC}$ \\
\hline J004216.08-725731.9 & $-1,91$ & $-8,58$ & $-5,66$ & $-5,41$ & $14,4^{\dagger}$ & $\mathrm{C}-\mathrm{N} 5$ \\
\hline J004456.46-731224.2 & $-3,50$ & $-7,76$ & $-4,91$ & $-4,64$ & 154,9 & $\mathrm{C}-\mathrm{J} 4$ \\
\hline J005058.90-730006.6 & $-2,82$ & $-8,02$ & $-5,15$ & $-4,92$ & 143,8 & $\mathrm{C}-\mathrm{N} 4,5$ \\
\hline J00 & $-3,08$ & $-7,55$ & $-4,72$ & $-4,55$ & 162,2 & $\mathrm{C}-\mathrm{J} 4$ \\
\hline J005401.40-731029.8 & $-2,99$ & $-7,65$ & $-4,81$ & $\ldots$ & 172,1 & $\mathrm{Ba} ?$ \\
\hline J00 & $-2,47$ & $-7,64$ & $-4,80$ & $-4,47$ & 114,2 & $\mathrm{C}-\mathrm{N} 5$ \\
\hline J005835.16-725935.4 & $-2,29$ & $-7,91$ & $-5,05$ & $-4,74$ & 129,2 & $\mathrm{C}-\mathrm{N} 5$ \\
\hline J005929.08-723926.7 & $-1,90$ & $-7,81$ & $-4,96$ & $-4,76$ & 88,7 & $\mathrm{C}-\mathrm{N} 5$ \\
\hline J010846.90-724019.4 & $-1,41$ & $-7,31$ & $-4,50$ & $\cdots$ & $11,8^{\dagger}$ & $\mathrm{C}-\mathrm{R} 5$ \\
\hline J050515.90-684446.6 & $-1,79$ & $-7,92$ & $-5,06$ & 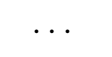 & 212,7 & $\mathrm{C}-\mathrm{N} 5$ \\
\hline J051139.99-684816.8 & $-3,05$ & $-7,31$ & $-4,50$ & $-4,02$ & 262,0 & $\mathrm{C}-\mathrm{N} 5$ \\
\hline J0515 & -2 & -6 & $-4,06$ & -3 , & 0 & 5 \\
\hline J051609.74-693517.9 & $-2,31$ & $-7,87$ & $-5,01$ & $\cdots$ & 222,2 & • \\
\hline J051810.88-692626.5 & $-4,87$ & $-7,90$ & $-5,04$ & $\cdots$ & $2307,9^{\dagger}$ & • \\
\hline J051954.02-692802.8 & $-3,33$ & $-7,08$ & $-4,28$ & $\cdots$ & 225,5 & $\mathrm{C}-\mathrm{N} 5$ \\
\hline J051958.26-691416.7 & $-3,63$ & $-7,46$ & $-4,63$ & $\cdots$ & $25,2^{\dagger}$ & $\mathrm{C}-\mathrm{N} 5$ \\
\hline J052047.00-695046.2 & $-2,72$ & $-8,44$ & $-5,53$ & 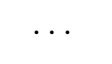 & 220,3 & $\mathrm{C}-\mathrm{N} 5$ \\
\hline J052154.24-705657.0 & $-1,60$ & $-7,10$ & $-4,30$ & $\cdots$ & 188,8 & $\mathrm{C}-\mathrm{N} 5$ \\
\hline J052555.11-694354.1 & $-2,43$ & $-7,66$ & $-4,82$ & $-4,45$ & 285,6 & $\mathrm{C}-\mathrm{N} 5$ \\
\hline J053424.83-694400.1 & $-4,90$ & $-6,18$ & $-3,46$ & $-4,14$ & 274,2 & $\mathrm{C}-\mathrm{H} 3$ \\
\hline J053608.58-702220.5 & $-2,44$ & $-7,68$ & $-4,84$ & $-4,40$ & 242,7 & $\mathrm{C}-\mathrm{N} 5$ \\
\hline J053900.00-695225.1 & $-6,64$ & $-8,70$ & $-5,77$ & $-5,68$ & $-99,5^{\dagger}$ & $\mathrm{C}-\mathrm{N} 4,5$ \\
\hline J053 & $-4,99$ & $-7,73$ & $-4,88$ & $-5,32$ & $193,7^{\dagger}$ & M8 III \\
\hline J054223.01-701810.3 & $-2,35$ & $-7,99$ & $-5,12$ & $\cdots$ & $193,2^{\dagger}$ & $\mathrm{C}-\mathrm{N} 5$ \\
\hline J054433. & $-2,89$ & $-8,26$ & $-5,37$ & $-5,08$ & 298,5 & $\mathrm{C}-\mathrm{N} 5$ \\
\hline J054658.32-702505.6 & $-2,64$ & $-8,03$ & $-5,16$ & $-4,72$ & 274,5 & $\mathrm{C}-\mathrm{N} 5$ \\
\hline
\end{tabular}

- indica espectro composto; $\dagger$ indica baixa probabilidade de pertencer às MC. 
radiação difusa, feitos em $100 \mu \mathrm{m}$, são uma combinação de outros obtidos a partir do IRAS (InfraRed Astronomy Satellite) e do DIRBE (Diffuse InfraRed Background Experiment, acoplado ao satélite COBE, COsmic Background Explorer).

A determinação do avermelhamento na direção das MC não é um problema com solução trivial. Apesar dos esforços, ainda não há um modelo que leve em conta todas as variáveis que determinam $\mathrm{R}_{V}$ na direção das $\mathrm{MC}$ (como por exemplo a distribuição diferencial de poeira dentro e fora da MW), de modo que a lei de extinção varia entre linear e não-linear de acordo com a linha de visada (Zagury, 2007). A princípio, é razoável dizer que o valor médio de $\mathrm{R}_{V}=3,1$ vale para todo o meio interestelar difuso; o trabalho de Rieke e Lebofsky (1985) favorece essa hipótese. Diante das dificuldades, é aceitável, ao menos como primeira aproximação, ter o meio interestelar Galáctico como único responsável pela extinção na direção das Nuvens. Portanto, optou-se pela utilização dos mapas de SFD98 neste trabalho para estimativas de avermelhamento.

O avermelhamento de SFD98 está disponibilizado eletronicamente pelos autores em forma de um programa ${ }^{7}$ em $\mathrm{IDL}^{8}$, onde fornecendo-se as coordenadas galácticas $(1, \mathrm{~b}$ ) obtém-se os valores de $\mathrm{E}(\mathrm{B}-\mathrm{V})$ da respectiva posição.

Arce e Goodman (1999) atentaram para uma superestimativa de um fator entre 1,3 e 1,5 no $\mathrm{E}(\mathrm{B}-\mathrm{V})$ de SFD98 para $\mathrm{A}_{V}>0,5$ na linha de visada. Esse trabalho é baseado em quatro técnicas diferentes de se medir a extinção na nuvem escura na região de Taurus, comparadas com os mapas de SFD98. Arce e Goodman atribuíram o valor superestimado de $\mathrm{E}(\mathrm{B}-\mathrm{V})_{S F D}$ à desafortunada escolha de 389 galáxias utilizadas no cálculo da conversão de densidade de coluna de poeira para $\mathrm{E}(\mathrm{B}-\mathrm{V})_{S F D} .90 \%$ dessas galáxias teriam baixos valores de avermelhamento, o que prejudicou a generalização de uma estimativa. Bonifacio et al. (2000) adotaram a correção da extinção dada pela Equação 2.3,

$$
E(B-V)=\left\{\begin{array}{cl}
E(B-V)_{S F D} & \text { para } E(B-V)_{S F D} \leq 0,10 \\
0,10+0,65 \cdot\left[E(B-V)_{S F D}-0,10\right] & \text { para } E(B-V)_{S F D}>0,10
\end{array}\right.
$$

Bessell e Brett (1988) estabeleceram relações de avermelhamento entre várias cores, dentre elas $\mathrm{E}(\mathrm{J}-\mathrm{K})$ e $\mathrm{E}(\mathrm{V}-\mathrm{K})$, com relação a $\mathrm{E}(\mathrm{B}-\mathrm{V})$. Dessa forma, pôde-se determinar as

\footnotetext{
${ }^{7}$ http://astro.berkeley.edu/ marc/dust/data/data.html.

${ }^{8}$ O trabalho fez uso do programa IDL e de sua Biblioteca de Uso em Astronomia (Landsman, 1993).
} 
correções de avermelhamento pelas Equações

$$
\begin{gathered}
E\left(J-K_{s}\right)=0,56 \cdot E(B-V)_{A G} \mathrm{e} \\
E\left(V-K_{s}\right)=2,78 \cdot E(B-V)_{A G} .
\end{gathered}
$$

Tal determinação fundamenta-se na hipótese de que $\mathrm{E}\left(\mathrm{J}-\mathrm{K}_{s}\right) \approx \mathrm{E}(\mathrm{J}-\mathrm{K})$ e $\mathrm{E}\left(\mathrm{V}-\mathrm{K}_{s}\right) \approx$ $\mathrm{E}(\mathrm{V}-\mathrm{K})$. Tal hipótese é apropriada pois o desvio característico entre as duas magnitudes é suficientemente pequeno (em média 0,026) para afetar as correções de avermelhamento. Os valores de avermelhamento $\mathrm{E}\left(\mathrm{J}-\mathrm{K}_{s}\right)$ e $\mathrm{E}\left(\mathrm{V}-\mathrm{K}_{s}\right)$ estão apresentados nas colunas 11 e 12 da Tabela 2.2. Daqui em diante, o índice "0" indica a cor corrigida pelo avermelhamento.

Os índices de cor $\left(\mathrm{J}-\mathrm{K}_{s}\right)_{0},(\mathrm{~J}-\mathrm{K})_{0}$ e $\left(\mathrm{V}-\mathrm{K}_{s}\right)_{0}$ para os objetos da amostra são apresentados nas colunas 16,17 e 18 da Tabela 2.2 .

Adotando um valor de $\mathrm{R}_{V}=3,12$ e $\mathrm{R}_{K}=0,34$ (Bessell e Brett, 1988), adequados para o meio interestelar difuso, calculou-se os valores de extinção nas bandas V e K através de:

$$
\begin{aligned}
& A_{V}=3,12 \cdot E(B-V) \mathrm{e} \\
& A_{K_{s}}=0,34 \cdot E(B-V),
\end{aligned}
$$

os quais são apresentados nas colunas 9 e 10 da Tabela 2.2. Assim como para o avermelhamento, é razoável fazer a aproximação $\mathrm{A}_{K_{s}} \approx \mathrm{A}_{K}$.

De acordo com SFD98, o valor da incerteza no avermelhamento em B-V é estimado como sendo da ordem de $16 \%$ do valor do índice de cor. Essa incerteza é propagada para $\mathrm{E}\left(\mathrm{J}-\mathrm{K}_{s}\right)$ e $\mathrm{E}\left(\mathrm{V}-\mathrm{K}_{s}\right)$, sendo portanto equivalente a $9 \%$ e $44 \%$ do avermelhamento em $\mathrm{J}-\mathrm{K}_{s}$ e $\mathrm{V}-\mathrm{K}_{s}$, respectivamente. Os avermelhamentos $\mathrm{A}_{V}$ e $\mathrm{A}_{K_{s}}$ apresentam incertezas de $50 \%$ e $5 \%$ de seu valor, nessa ordem.

\subsection{Magnitudes visuais aparente e absoluta}

As magnitudes visuais aparente ( $\mathrm{V}$, de acordo com o sistema fotométrico padrão) e absoluta $\left(\mathrm{M}_{V}\right)$ das estrelas foram obtidas a partir da base de dados do catálogo Simbad. Foram encontradas as magnitudes $\mathrm{V}$ para apenas sete das 28 estrelas que constituem a amostra de candidatas a CEMP e para duas estrelas-padrão observadas ao longo da campanha. Com exceção das estrelas-padrão, cujas magnitudes foram fornecidas por Mermilliod (1986), os demais objetos fazem parte do catálogo de estrelas C da SMC cuja fotometria foi medida por Rebeirot et al. (1993). Com as magnitudes V e os espectros (calibrados 
em fluxo) das estrelas da amostra, foi possível derivar as magnitudes visuais aparentes dos objetos restantes.

Nesse sentido, foi utilizada, para cada objeto, a seguinte equação na calibração da curva aplicada para o cálculo de $\mathrm{V}$ dos outros objetos:

$$
V=-2,5 \log \left(F_{V}\right)+C
$$

onde $\mathrm{F}_{V}$ representa o fluxo integrado no intervalo de comprimentos de onda da banda $\mathrm{V}$, dado em erg/s/ $\mathrm{cm}^{2}$. O fluxo $\mathrm{F}_{V}$ foi ponderado pela curva de transmissão do filtro $\mathrm{V}$ de Johnson-Cousins (Johnson e Morgan, 1953).

O valor da constante $\mathrm{C}$ foi calculado para cada um dos objetos de calibração. A partir desse resultado foi calculada a C média, valor utilizado no cálculo da magnitude V para os objetos restantes, a partir da Equação 2.6. Os dados utilizados e resultantes da calibração (constante $\mathrm{C}, \mathrm{V}$ de calibração calculado e seu desvio padrão) são apresentados na Tabela 2.6. Os valores de $\mathrm{C}$ médio e do desvio padrão da média encontrados foram

$$
C=-14,23 \pm 0,31
$$

A incerteza final de cada magnitude $\mathrm{V}$ é dada pela equação

$$
\sigma_{V}^{2}=\left[-2,5\left(1+\frac{1}{F_{V} \ln 10}\right) \log \left(F_{V}\right)\right]^{2} \sum_{\lambda}\left(\Delta \lambda \sigma_{F_{\lambda}}\right)^{2}+\sigma_{C}^{2},
$$

onde $\sigma_{F_{\lambda}}$ é a incerteza do fluxo calibrado em cada comprimento de onda, espaçados em $\Delta \lambda=1,5$ em todos os espectros. A média das incertezas em $\mathrm{V}$ é 0,33 , sendo que a principal contribuição para esse valor é a incerteza da constante C.

As magnitudes V calculadas são apresentadas na coluna 3 da Tabela 2.2. Nessa Tabela, as magnitudes V de calibração são indicadas pelo símbolo *. A Figura 2.8 mostra um gráfico de resíduos absolutos, ou subtração entre $\mathrm{V}$ calculadas $\left(\mathrm{V}_{\text {calc }}\right)$ e das estrelas-padrão $\left(\mathrm{V}_{\text {pad }}\right)$. Tal Figura mostra que a maior diferença entre esses dois valores é maior que a unidade apenas no caso de 3 objetos. $\mathrm{O}$ índice de correlação $r$ entre $\mathrm{V}_{\text {calc }}$ e $\mathrm{V}_{\text {pad }}$ é 0,97. Tais resultados mostram uma forte correlação entre as magnitudes $V_{\text {pad }}$ e $V_{\text {calc }}$ e uma dispersão que é típica de estrelas variáveis, o que não compromete a validade dos dados. Os valores de $\mathrm{V}_{\text {calc }}$ são, portanto, confiáveis e adequados para futura análise.

A partir da equação de módulo de distância,

$$
V-M_{V}-A_{V}=\mu
$$


Tabela 2.4 - Informações das estrelas consideradas como padrões no cálculo de V dos objetos da amostra.

\begin{tabular}{ccccc}
\hline \hline Objeto & $\mathrm{V}_{\text {pad }}$ & $\mathrm{C}$ & $\mathrm{V}_{\text {calc }}$ & $\sigma_{V}$ \\
\hline OGLE J003722.15-732222.8 & 16.69 & -13.82 & 16.27 & 0.31 \\
OGLE J004216.08-725731.9 & 17.41 & -15.33 & 18.50 & 0.34 \\
OGLE J005058.90-730006.6 & 17.11 & -14.94 & 17.82 & 0.32 \\
OGLE J005106.53-730502.8 & 16.80 & -14.04 & 16.60 & 0.31 \\
OGLE J005516.51-725733.4 & 16.94 & -15.86 & 18.57 & 0.38 \\
OGLE J005835.16-725935.4 & 17.02 & -14.31 & 17.09 & 0.31 \\
OGLE J005929.08-723926.7 & 17.60 & -13.71 & 17.07 & 0.31 \\
LTT 377 & 10.60 & -13.39 & 9.76 & 0.31 \\
GD 71 & 13.06 & -12.72 & 11.54 & 0.31 \\
\hline
\end{tabular}

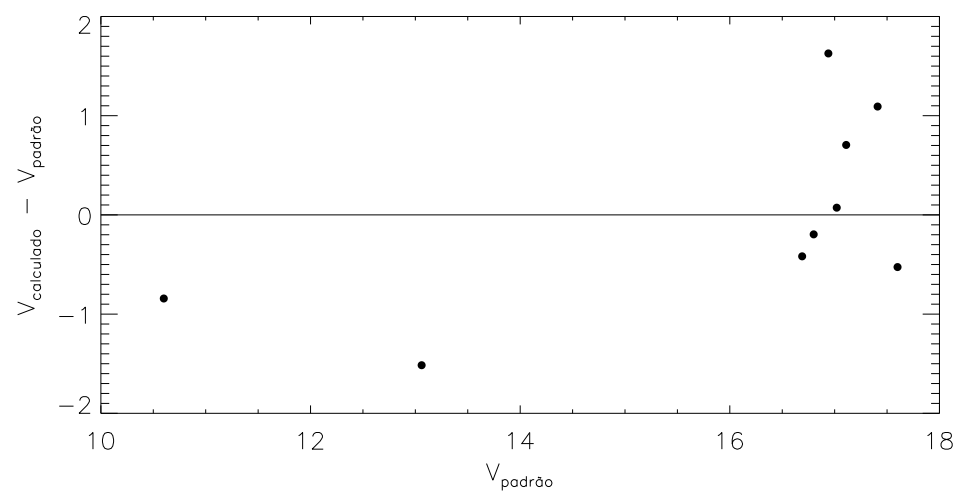

Figura 2.8: Comparação entre V calculado e V fornecido pela literatura das estrelas-padrão.

dos valores de $\mathrm{A}_{V}$ (ver Seção 2.5) e dos módulos de distâncias ( $\mu$ ) conhecidos para LMC e SMC, pôde-se calcular as magnitudes absolutas $\mathrm{M}_{V}$ para cada objeto da amostra. $\mathrm{O}$

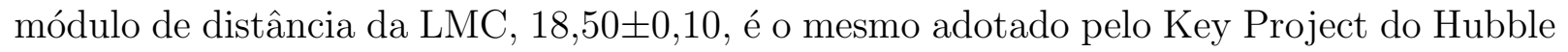

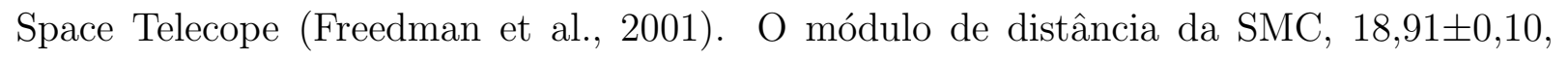
foi determinado por Hilditch et al. (2005); esse valor resultou do estudo de uma amostra contendo 40 sistemas binários eclipsantes. Os valores de $\mathrm{M}_{V}$ são apresentados na coluna 2 da Tabela 2.3. As magnitudes absolutas $\mathrm{M}_{K}$, calculadas pela equação

$$
M_{K}=K-A_{K}-\mu,
$$

são apresentadas na coluna 3 da Tabela 2.3. A incerteza média de $\mathrm{M}_{V}$ e $\mathrm{M}_{K}$, dadas pela 
soma quadrática das incertezas relativas a cada termo das equações 2.9 e 2.10, são 0,80 e 0,15 , respectivamente.

\subsection{Estimativa de magnitudes bolométricas}

Há na literatura relações que permitem calcular magnitudes bolométricas de populações associadas a estrelas C. Wood et al. (1983) forneceram a correção bolométrica $\mathrm{BC}_{K}$ para estrelas LPV do centro da MW e Miras da mesma localidade do Sol, dada pela equação

$$
M_{b o l}^{W}=M_{K}+B C_{K}
$$

onde, para $0,6<(\mathrm{J}-\mathrm{K})_{0}<2,0$,

$$
\begin{gathered}
B C_{K}=0,69+2,65(J-K)_{0}-0,67(J-K)_{0}^{2} \text { na LMC e } \\
B C_{K}=0,55+2,65(J-K)_{0}-0,67(J-K)_{0}^{2} \text { na SMC }
\end{gathered}
$$

Neste caso, (J-K) $)_{0}$ é dada pelo sistema de Bessell e Brett (1988). Alksnis et al. (1998) forneceu uma calibração de $\mathrm{M}_{\text {bol }}$ baseada em estrelas $\mathrm{C}$ da MW, com distâncias determinadas a partir de medições de paralaxes realizadas pela missão astrométrica Hipparcos (Turon et al., 1996), dada pela equação

$$
M_{b o l}^{A}=2,23+0,92 M_{K}
$$

Nenhuma dessas equações pode ser utilizada indiscriminadamente no cálculo das magnitudes bolométricas de estrelas C das MC. No entanto, ainda servem para uma estimativa e posterior comparação das estrelas da amostra aqui estudada com resultados de outros trabalhos. Não obstante, os valores encontrados pelas duas equações (colunas 4 e 5 da Tabela 2.3) são muito similares, o que torna essas estimativas adequadas ao menos para uma análise qualitativa.

\subsection{Velocidades radiais}

As velocidades radiais foram calculadas pelo método de correlação cruzada (Tonry e Davis, 1979), onde se compara o espectro observado a um espectro modelo (template). A partir da distribuição de desvios de correlação de pontos (no caso comprimentos de onda, relacionados diretamente a velocidades), obtém-se a velocidade mais provável do 
objeto. Sivarani (comunicação particular) utilizou um programa construído em IDL para realizar o cálculo de tais velocidades. Também com uma rotina em IDL, as velocidades observadas foram então corrigidas para o referencial centrado no Sol. As velocidades radiais heliocêntricas são apresentadas na coluna 6 da Tabela 2.3. A incerteza média das velocidades radiais heliocêntricas das estrelas é de $10 \mathrm{~km} / \mathrm{s}$, estimativa extraída da largura a meia altura das distribuições de velocidades.

Apesar da amostra ter sido observada no campo visual das MC, é importante verificar se os objetos observados fazem parte realmente das Nuvens e não de outros sistemas como, por exemplo, o halo da MW. A execução dessa tarefa é alcançada comparando-se as velocidades radiais heliocêntricas dos objetos com a velocidade típica das Nuvens: quanto mais próxima a velocidade do objeto estiver da velocidade das MC, maior a probabilidade de pertencer à respectiva galáxia.

As velocidades das estrelas de cada Nuvem correspondem à soma de diversas componentes relativas à composição de seus movimentos com relação à MW, como translação, rotação, precessão e nutação. Apesar da complexidade de informações agregadas à determinação da velocidade desses objetos, há um comportamento sistêmico dos dados, de forma que a velocidade do centro de massa da Nuvem deve se sobressair das outras componentes; assim, a velocidade radial deve ser uma projeção da velocidade do centro de massa na linha de visada. Segundo van der Marel et al. (2002), a velocidade radial sistêmica (na linha de visada de um observador centrado no Sol) da população de estrelas C da LMC e a respectiva dispersão $(\sigma)$ é

$$
v_{\text {rad }}=262,2 \pm 3,4 \mathrm{~km} / \mathrm{s}, \sigma=20,2 \pm 0,5 \mathrm{~km} / \mathrm{s} .
$$

De acordo com Hatzidimitriou et al. (1997), a velocidade radial da população de estrelas C da SMC é

$$
v_{\text {rad }}=149,3 \pm 3,0 \mathrm{~km} / \mathrm{s}, \sigma=25,2 \pm 2,1 \mathrm{~km} / \mathrm{s} .
$$

É razoável a utilização da dispersão de velocidades como parâmetro para delimitar o espaço de velocidades no qual há maior probabilidade de encontrarmos uma estrela C que pertença às Nuvens. A suposição de que velocidades radiais de estrelas C da MC sejam inferiores a $3 \sigma$ é razoável para um estudo preliminar acerca da amostra de candidatas a estrelas C. 
As estrelas com baixa probabilidade de pertencerem às MC, de acordo com o critério apresentado, são assinaladas com o símbolo $†$ na coluna 6 da Tabela 2.3. É importante ressaltar que a incompatibilidade das velocidades dessas estrelas com relação às das Nuvens não significa que não pertençam às MC: estrelas de alta velocidade, que são comuns nas MC, e objetos binários podem sofrer grandes variações de velocidades radiais, causando a falsa impressão de que não fazem desse sistema. 


\section{Capítulo 3}

\section{Análise dos dados}

\subsection{Derivação dos parâmetros atmosféricos de estrelas CEMP}

A derivação dos parâmetros atmosféricos das estrelas da amostra é um passo fundamental para sua caracterização, o que viabiliza sua comparação com as populações estelares tanto da MW quanto das MC. É nesse contexto em que se insere a necessidade de um método que, baseado em informações fotométricas e principalmente espectroscópicas, seja capaz de fornecer as informações acerca das abundâncias elementais entre outras características das atmosferas estelares.

Muitos estudos sobre abundância espectroscópica de alta resolução de estrelas MP veem sendo desenvolvidos (McWilliam et al., 1995; Cayrel et al., 2004; François et al., 2007) e seus resultados têm mostrado que os padrões de abundância das estrelas MP divergem dos do Sol. Em particular, está bem estabelecido que a razão de elementos $\alpha$ em relação ao ferro $([\alpha / \mathrm{Fe}])$ em estrelas com $[\mathrm{Fe} / \mathrm{H}]<-1,0$ é maior que a razão solar em $0,4 \operatorname{dex}$ (Wheeler et al., 1989), o que indica a complexidade da natureza do enriquecimento químico em sistemas estelares.

Entretanto, as fontes primárias de nucleossíntese da maioria dos elementos não são

bem conhecidas. A razão relativa de abundâncias, ou seja, a razão de abundância entre algum elemento e o ferro, tem sido uma ótima ferramenta para a investigação do sítio de produção de cada elemento. No entanto, as inomogeneidades nas abundâncias resultantes de diversos estudos, exibindo espalhamentos e desvios expressivos, têm sido recorrentes. Isso se deve a vários fatores: para se determinar a abundância de um determinado elemento, os parâmetros atmosféricos - temperatura efetiva $\left(\mathrm{T}_{\text {eff }}\right)$, gravidade superficial $(\log g)$, velocidade de microturbulência $(\mu)$ e metalicidade - precisam ser derivados; além 
disso, diferenças nos resultados desses parâmetros devem-se a peculiaridades na atmosfera, que são dependentes das abundâncias químicas; outro fator relevante é a escolha das linhas consideradas na análise e valores das forças de oscilador $(\log g f)$ adotados na derivação das abundâncias, os quais acarretam incertezas sistemáticas nos resultados; por fim, mas não encerrando o problema, Ryan et al. (1996) mostraram que ignorar a estrutura eletrônica hiperfina pode levar a erros na abundância de até 0,6 dex. Tais fatores exigem a determinação de abundâncias de elementos químicos nas estrelas em um amplo intervalo de metalicidades, tão acurada quanto possível e utilizando métodos homogêneos, a fim de se obter pistas sobre suas origens ainda não descobertas.

A estrutura da atmosfera estelar é dependente da composição química devido à contribuição dos metais na opacidade no contínuo espectral: conforme cresce a concentração de metais, cresce também a pressão eletrônica como resultado do maior número de elétrons livres, que, por seu turno, afeta a temperatura e a pressão diferencial da atmosfera. Dentro do intervalo de temperatura de interesse, metais como C, $\mathrm{Si}, \mathrm{Al}, \mathrm{Mg}$ e Fe contribuem para a maior parte da opacidade no contínuo. Espera-se que as atmosferas das estrelas CEMP, com suas altas abundâncias de $\mathrm{C}$, tenham perfis de temperatura e pressão diferentes das estrelas MP com abundância "normal" de carbono. Sob essa perspectiva, a utilização de modelos de atmosferas estelares construídos com base em abundâncias do tipo solar em análises do conteúdo de estrelas CEMP pode provocar estimativas inapropriadas de abundâncias, devido à inconsistência na estrutura de temperatura e pressão das regiões de formação das linhas por conta do enriquecimento em carbono.

Infelizmente, modelos de atmosferas de estrelas enriquecidas em carbono ainda não estão tão amplamente disponibilizados quanto os de abundâncias do tipo solar (Castelli e Kurucz, 2004; Gustafsson et al., 2008); por isso, não é surpresa os estudos sobre estrelas CEMP terem se apoiado em abundâncias deste tipo. Ao menos uma grande parte desses estudos comparou modelos enriquecidos em carbono com os de tipo solar (Lucatello et al., 2003; Aoki et al., 2007). Em especial, Hill et al. (2000), em uma investigação mais específica, mostrou que as camadas superiores das atmosferas enriquecidas em carbono, onde normalmente se formam as linhas mais fortes, são mais frias que em atmosferas com abundância solar, enquanto suas camadas internas, onde são formadas as linhas mais fracas, são mais quentes. Essas diferenças na estrutura de temperatura devem afetar as 
derivações de abundância, especialmente aquelas baseadas em características moleculares, mais sensíveis à temperatura.

Problemas com estimativa de abundâncias químicas não residem somente na inexistência de modelos de atmosferas adequados. Para estrelas MP, o método espectroscópico pode não ser satisfatório devido à falta das linhas de metais necessárias no espectro. Embora a melhor forma de se derivar abundâncias químicas seja a espectroscopia de alta resolução, tal técnica requer uma enorme quantidade de tempo em grandes telescópicos para se medir um número significativo de estrelas, mesmo em galáxias mais próximas. A alternativa é a espectroscopia de baixa resolução que, em união com os modernos espectrógrafos multiobjetos, permite a observação de grandes amostras em tempo razoável. Entretanto, em galáxias externas, baixa resolução é viável apenas para os objetos mais brilhantes, na maioria dos casos, estrelas do ramo das gigantes vermelhas (RGB, red giant branch) ou em fases evolutivas mais avançadas.

\subsubsection{O método de Sivarani et al. (2008)}

Sivarani et al. (2008, SBL08) vêm desenvolvendo um método automatizado para estimar uma série de parâmetros atmosféricos, a ser aplicado nos objetos selecionados do catálogo de estrelas CEMP de Christlieb et al. (2001), fornecido pelo Hamburg/ESO Survey.

O método baseia-se em uma grade de parâmetros atmosféricos obtidos a partir de espectros sintéticos (espectroscopia óptica, 3800-4800Å, de média resolução, $\mathrm{R}=2000$ ) os quais utilizam uma versão do código MARCS (Gustafsson et al., 2003) adaptado por Bertrand Plez e Thomas Masseron. Esse código é particularmente interessante para as estrelas do tipo CEMP por dar destaque a uma estrutura atmosférica que varia com o aumento da abundância de carbono (Masseron et al., 2006). A grade é composta por 2000 espectros de atmosferas enriquecidas em carbono e cobre os intervalos de $4000<\mathrm{T}_{\text {eff }}(\mathrm{K})$ $<6000$, de $0,0<\log g<5,0$ e $0,0<[\mathrm{C} / \mathrm{Fe}]<4,0$. As abundâncias de nitrogênio são admitidas 0,5 dex menores que as de carbono, o que de acordo com SBL08 é apropriado para estrelas CEMP. A lista de linhas de CH e CN são as mesmas compiladas por Plez e Cohen (2005), enquanto a lista de linhas de $\mathrm{C}_{2}$ são as de Kurucz (1993).

A estimativa dos parâmetros segue os seguintes passos: após reduzir a grade sintética à 
resolução do espectro observado $(\mathrm{R}=2000)$, é feita a minimização por $\chi^{2}$, usando a rotina de minimização multidimensional ou método de downhill simplex (Nelder e Mead, 1965) do IDL. As temperaturas são estimadas seguindo as calibrações de Alonso et al. (1996) com base em índices de cor $\mathrm{V}-\mathrm{K}$ e J-K. A calibração de Alonso et al. em V-K requer cores no sistema fotométrico padrão de Johnson (Johnson, 1963), enquanto a calibração em J-K foi feita com base no sistema TCS (o sistema fotométrico nativo do telescópio Carlos Sánchez em Tenerife; Arribas e Roger 1987). Para a obtenção das cores V-K no sistema de Johnson, a magnitude $\mathrm{K}_{s}$ obtida do 2MASS foi transformada ao sistema de Bessell e Brett (1988) utilizando-se as equações do Complemento Explanatório do 2MASS; vale ressaltar que essa calibração segue dois passos, do sistema do 2MASS para o CIT (Burstein e Heiles, 1982; Frogel et al., 1978) e então para o sistema de Bessel e Brett. Portanto, os erros envolvidos nas transformações de cores devem ser combinados com os associados à fotometria. Já para transformar as cores $\mathrm{J}-\mathrm{K}_{s}$ do 2MASS em sistema TCS de acordo com Alonso et al. (1994), novamente foi necessária uma transformação em dois passos, do sistema do 2MASS para o CIT e do CIT para o TCS. A minimização do ajuste levando em conta todos os parâmetros é conduzida pela razão $\mathrm{C} / \mathrm{O}$, uma vez que as absorções das moléculas de $\mathrm{CN}, \mathrm{C}_{2}$ e $\mathrm{CH}$ são as características mais marcantes do espectro. As metalicidades são determinadas pelo ajuste da linha K do CaII, enquanto o $\log g$ é derivado pelo ajuste da linha $\lambda 4427$ do CaI. Todos os parâmetros são revisados iterativamente até o melhor ajuste ser atingido.

O método de SBL08 baseia-se em estrelas que são relativamente mais quentes que as da amostra aqui estudada - o intervalo de temperaturas da grade não corresponde às temperaturas assinaladas pelos tipos espectrais das estrelas da amostra (Seção 3.2.2). De acordo com Sivarani (comunicação particular), no limite inferior de $\mathrm{T}_{\text {eff }}$, o método deveria apresentar resultados razoáveis, pois na realidade sua grade considera estrelas com temperaturas inferiores a 4000K, próximas a 3900K. Como ainda não existe um método que forneça parâmetros atmosféricos de estrelas tão frias, tentou-se utilizar o método de SBL08 na estimativa de $\mathrm{T}_{e f f}, \log g,[\mathrm{Fe} / \mathrm{H}]$ e $[\mathrm{C} / \mathrm{Fe}]$. No entanto, as temperaturas fornecidas pelo método foram inferiores até mesmo que 3900K. Assim, as temperaturas das estrelas da amostra são incompatíveis com SBL08, tornado as estimativas inadequadas para serem comparadas aos dados referentes a populações estelares da MW e MC. 


\subsection{Classificação espectral}

\subsubsection{Sistema MK Revisado}

A classificação espectral de estrelas C tem como objetivo aumentar a acurácia da comparação das estrelas de carbono individuais em diferentes regiões da MW e galáxias vizinhas. O principal sistema de classificação espectral de estrelas C, o MK93 (ver Seção 1.3), funciona não apenas no sentido de ilustrar a separação dos objetos estelares de acordo com suas afinidades, mas também no de distinguir as propriedades decorrentes da evolução estelar das determinadas pelo meio ambiente. Embora tenha respaldo em um processo de classificação que não considera hipóteses físicas, a definição dos tipos espectrais subsidia a elaboração de tais hipóteses. A classificação espectral é, portanto, fundamental por dar condições à descoberta da origem da sobreabundância do carbono, atualmente associada de forma não muito clara tanto a fontes internas quanto externas às estrelas.

Atmosferas estelares frias e enriquecidas em carbono possuem fluxo na região entre 4000 e $10000 \AA$ drasticamente reduzido pela absorção por moléculas de $\mathrm{C}_{2}$, $\mathrm{CH}$ e $\mathrm{CN}$; essas são as características visuais imediatas dos espectros de estrelas $\mathrm{C}$ em geral. No entanto, de acordo com o Sistema MK93, há uma série de detalhes a serem considerados na distinção dos diversos tipos espectrais, o que requer uma investigação minuciosa das linhas de absorção e emissão.

A classificação de MK93 foi apoiada em espectrogramas fotográficos e traçadores microfotômetros com uma resolução de aproximadamente $3 \AA \AA$ no azul. Nessa classificação, as estrelas C se dividem nos tipos espectrais C-R, C-J, C-N, C-H e C-Hd.

As estrelas de tipo espectral C-R, as mais quentes dentre as estrelas $\mathrm{C}$, possuem abundâncias de elementos $s$ muito próximas à solar.

O tipo espectral C-J, caracterizado principalmente pela alta abundância de ${ }^{13} \mathrm{C}$, possui uma série de propriedades de fácil identificação visual em seu espectro. Na região vermelha, a absorção nas bandas de $\mathrm{C}_{2}$ revela a abundância dos isótopos de carbono. As bandas de Merrill-Sanford de $\mathrm{SiC}_{2}$, localizadas entre 4500 e $5000 \AA$, são encontradas com frequência nesse tipo espectral. Há ainda na região azul a banda de ${ }^{12} \mathrm{C}^{13} \mathrm{C}$ em $\lambda 4752$, embora seu reconhecimento seja prejudicado pela recorrente saturação da região.

As estrelas de tipo espectral C-N são normalmente enriquecidas em elementos $s$ (parti- 
cularmente Ba) ao contrário das de tipo C-R e C-J. A região azul de seus espectros muitas vezes apresenta fluxo nulo. Suas bandas isotópicas são fracas, em contraste com as de tipo C-J. As estrelas C-N se assemelham às C-J com relação à presença de bandas de Merrill-Sanford de $\mathrm{SiC}_{2}$, apesar da menor frequência dessas bandas neste tipo espectral.

O tipo espectral C-H é associado a estrelas que possuem forte absorção nas bandas de $\mathrm{CH}$ na parte azul do espectro. Elas se diferenciam das C-R por apresentarem maiores abundâncias de elementos $s$.

As estrelas C-Hd (deficientes em hidrogênio) possuem bandas de absorção de $\mathrm{CN}_{\mathrm{N}} \mathrm{C}_{2}$; no entanto, a banda $\mathrm{G}$ de $\mathrm{CH}$ é ausente ou muito fraca quando comparada à do tipo $\mathrm{C}-\mathrm{H}$.

Barnbaum et al. (1996) compilaram em um atlas espectros de estrelas C representativas

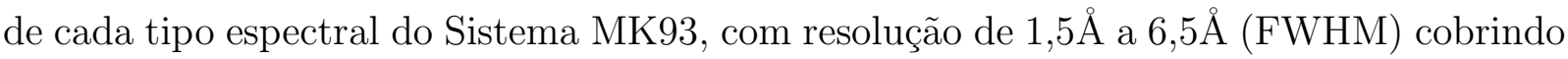
um intervalo de comprimento de onda de 4000 a $7000 \AA$. Esse atlas, que engloba sua versão anterior (Barnbaum, 1994), é composto basicamente por estrelas C de classes de luminosidade diferentes (Gray e Corbally, 2009) além de apresentar amostras de estrelas de Ba, cujos espectros possuem propriedades comuns aos de estrelas C. Os critérios utilizados na classificação levaram em consideração o índice $\mathrm{C}_{2}$, uma medida da razão das bandas de Swan de $\mathrm{C}_{2}$; o índice isotópico $j$, usado como medida da razão ${ }^{12} \mathrm{C}^{13} \mathrm{C}$ (Yamashita, 1972); e outros índices de medida de abundâncias moleculares, como de CH. A determinação dos índices foi feita visualmente, atribuindo a eles valores de 1 a 5 .

\subsubsection{Classificação da amostra de candidatas a CEMP}

As estrelas da amostra de candidatas a CEMP tiveram seus espectros comparados visualmente com os presentes no atlas de Barnbaum et al. (1996). Os critérios considerados na classificação das estrelas da amostra foram definidos de acordo com as principais características dos distintos tipos espectrais das estrelas C, de acordo com o Sistema MK93:

- C-J - ausência de linhas de BaII (representante do processo s), presença de bandas de ${ }^{13} \mathrm{CN}$ em $\lambda 6260$, forte absorção na banda de ${ }^{12} \mathrm{C}^{13} \mathrm{C}$ em $\lambda 6168$ com relação à de ${ }^{12} \mathrm{C}^{12} \mathrm{C}$ em $\lambda 6122$ e eventual presença de bandas de Merrill-Sanford de $\mathrm{SiC}_{2}$ em $\lambda \lambda 4540,4639,4802,4909$ e 4977;

- C-N - presença de linhas de BaII em $\lambda \lambda 4554,4934$ e 6497, bandas relativamente fracas de ${ }^{12} \mathrm{C}^{13} \mathrm{C}$ e eventual presença de bandas de Merrill-Sanford de $\mathrm{SiC}_{2}$; 
- C-H - absorção na banda G de CH, próxima a $\lambda 4300$;

- C-R - ausência de linhas de BaII, de bandas de Merrill-Sanford de $\mathrm{SiC}_{2}$ e bandas relativamente fracas de ${ }^{12} \mathrm{C}^{13} \mathrm{C}$.

- outros - propriedades que não se enquadram em nenhum dos tipos espectrais anteriores ou típicos de outras classes espectrais não contempladas por MK93.

A Tabela 2.3 apresenta em sua coluna 7 os tipos espectrais dos objetos da amostra, resultado da comparação visual com o atlas. Nas Figuras 3.1 até 3.4 são apresentados exemplos dos detalhes dos espectros considerados na comparação entre os objetos da amostra e os presentes no atlas de Barnbaum e colaboradores.

\subsection{Identificação de estrelas $C$ por critérios fotométricos}

Diagramas baseados em cores e magnitudes são ferramentas convenientes para a seleção de determinadas populações estelares inseridas em uma dada amostra.

Cioni et al. (2003) selecionaram estrelas C das MC com base em suas cores, restringindoas pelo limite $\mathrm{J}-\mathrm{K}_{s} \geq 1,33$. De acordo com Cioni et al. (2000), estrelas AGB evoluídas, que ocupam a região do topo do RGB, possuem $\mathrm{K}_{s} \leq 12,6$ e $\mathrm{J}-\mathrm{K}_{s} \gtrsim 0,97$. Raimondo et al. (2005) estudaram estrelas C pulsantes da SMC utilizando diagramas cor-cor (J- $\mathrm{K}_{s}$ vs. $\left.\mathrm{K}_{s}\right)$ e cor-magnitude $\left(\mathrm{H}-\mathrm{K}_{s}\right.$ vs. J-H), alterando o intervalo no qual se pode garantir a presença de estrelas $\mathrm{C}$ para $\mathrm{K}_{s}<12$; esse trabalho estabeleceu $\mathrm{J}-\mathrm{K}_{s}>1,33$ e $\mathrm{K}_{s}<12$ como os limites da região onde residem as estrelas C. Os critérios não levam em consideração avermelhamento interestelar. Marigo e Girardi (2005) reconheceram que em $\mathrm{J}-\mathrm{K}_{s}=1,33$ é o local onde residem as estrelas na terceira fase do AGB.

Na Figura 3.5 é apresentado o diagrama cor-magnitude $J-K_{s}$ vs. $\mathrm{K}_{s}$ (sem correção de avermelhamento para que a comparação seja consistente com as referências citadas). Triângulos representam objetos presentes na SMC e quadrados indicam objetos da LMC. Os objetos localizados entre as linhas azuis estão de acordo com o critério fotométrico que apontam estrelas C, seguindo Raimondo et al. (2005). Os dois objetos além dessa região são OGLE J004032.22-732048.3 e OGLE J053424.83-694400.1. Os dois objetos, de acordo com a classificação espectral, são de tipos dC e C-H, respectivamente. 


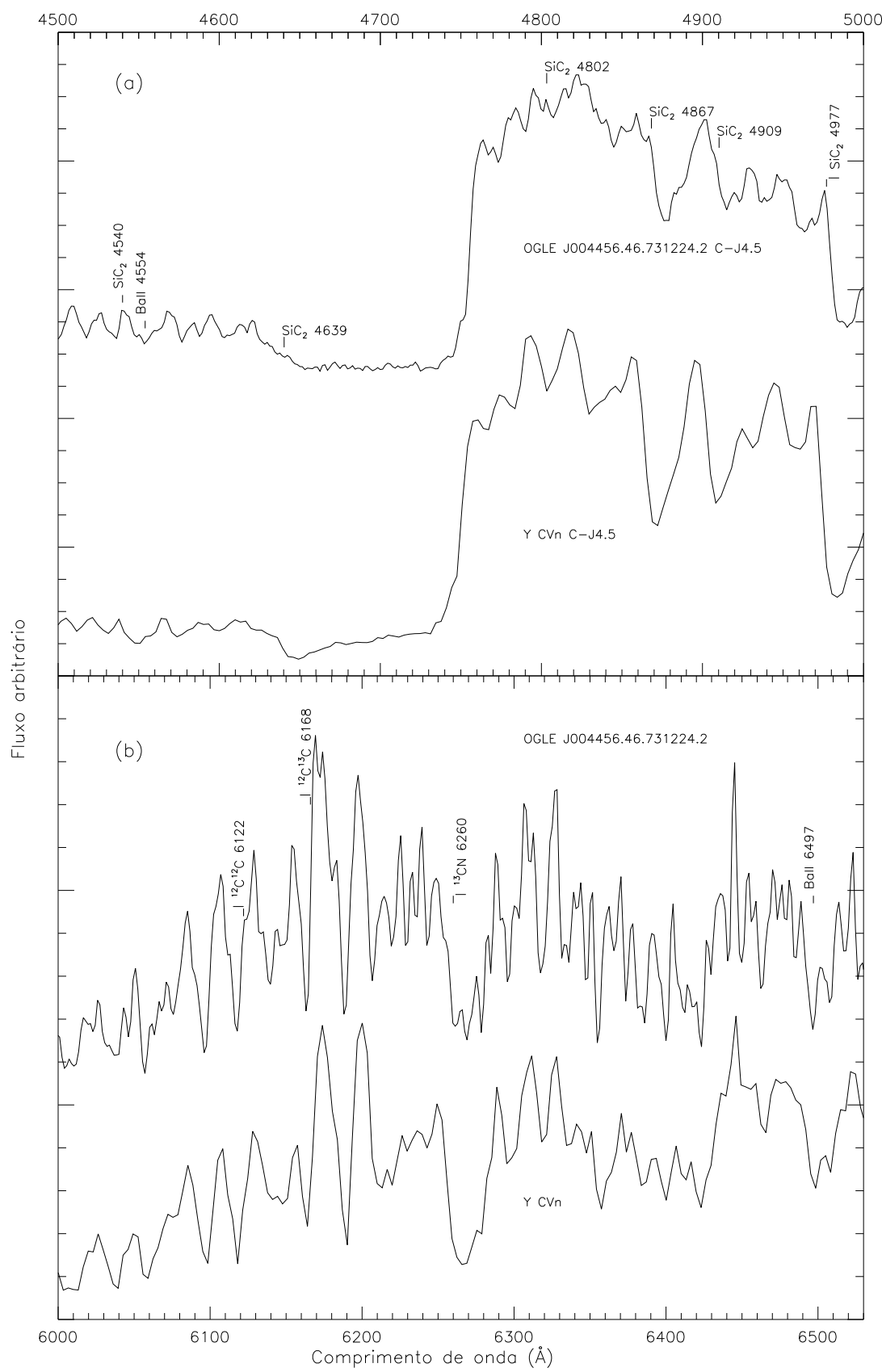

Figura 3.1: Detalhes das semelhanças entre objetos de tipo espectral C-J nas regiões azul (a) e vermelha (b) do espectro.

Na Fig. 3.6 é apresentado o diagrama cor-cor $\mathrm{H}-\mathrm{K}_{S}$ vs. J-H. Os objetos à direita da linha diagonal $\left(\mathrm{J}-\mathrm{K}_{s}>1,33\right)$ são os apontados como estrelas C (Cioni et al., 2003). A região delimitada pelo quadrado azul, no canto superior direito do diagrama $\left(\mathrm{H}-\mathrm{K}_{s}>0,8\right.$ e $\mathrm{J}-\mathrm{H}>1,2$ ), indica os objetos obscurecidos em fase AGB. O brilho reduzido dos objetos nessa área do diagrama pode ser consequência do estágio evolutivo mais avançado que o 


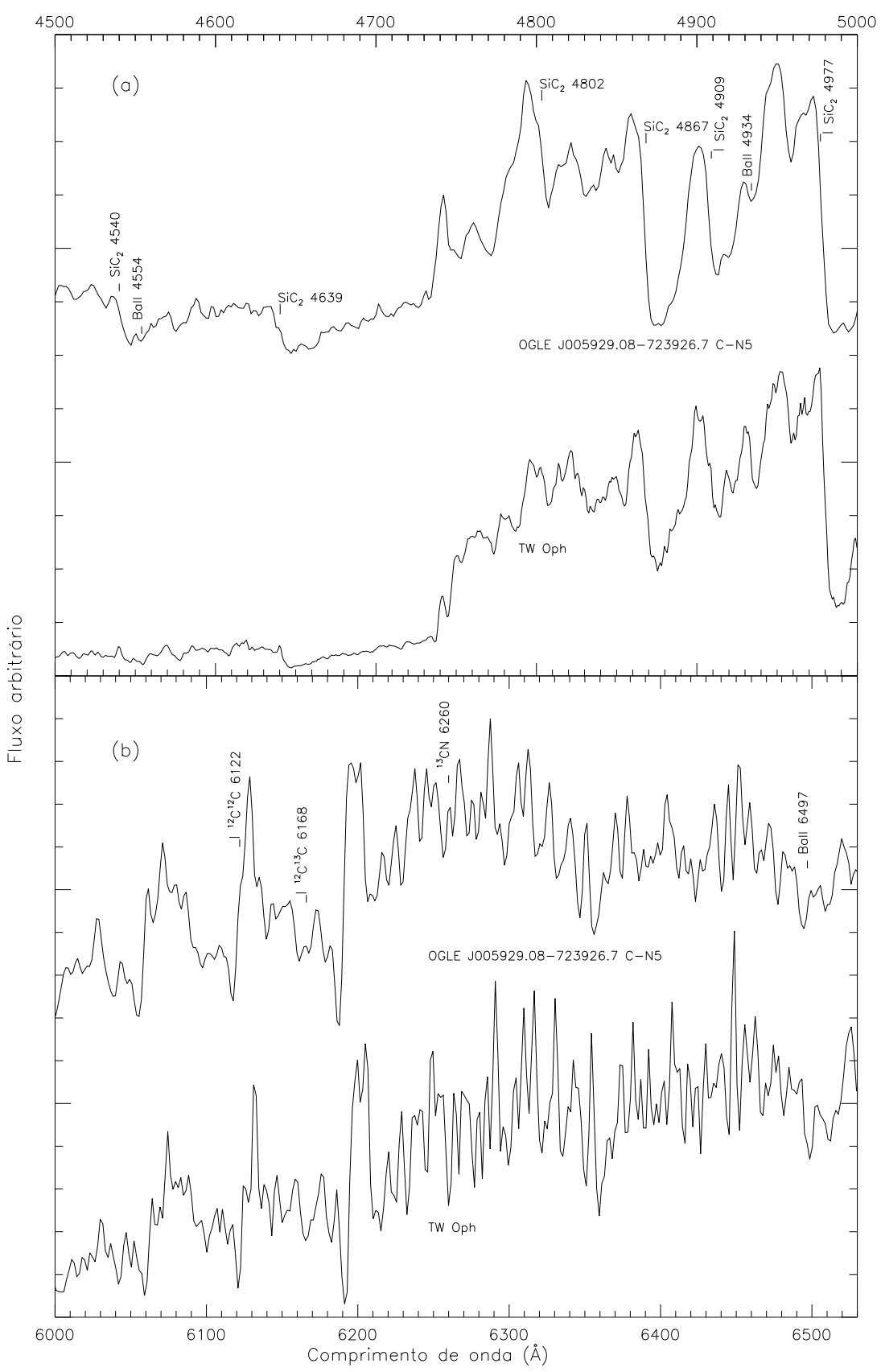

Figura 3.2: Detalhes das semelhanças entre objetos de tipo espectral C-N nas regiões azul (a) e vermelha (b) do espectro.

das estrelas relativamente mais brilhantes. Outra justificativa pode ser a observação desses objetos em uma fase de mínimo de luminosidade (Westerlund et al., 1995).

Os dois objetos abaixo da linha diagonal da Fig. 3.6 são OGLE J004032.22-732048.3 (dC) e OGLE J053424.83-694400.1 (C-H). Ambos se destacam também na Fig. 3.5. O motivo de serem mais azuis do que os outros objetos, fato que provoca sua distinção fo- 


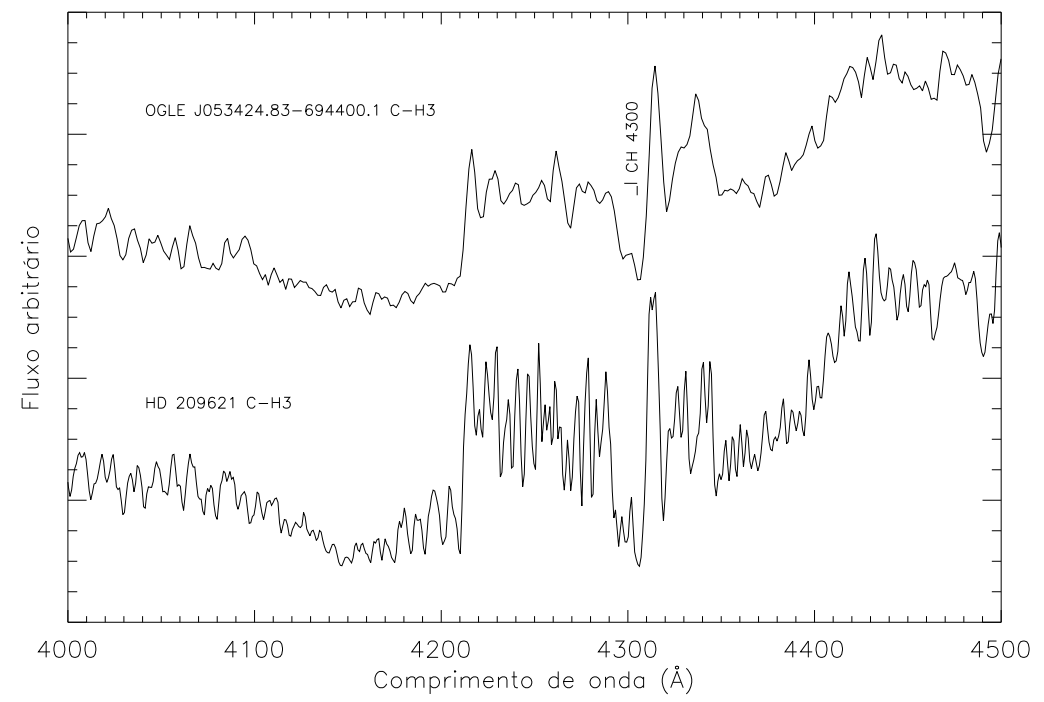

Figura 3.3: Detalhes das semelhanças entre objetos de tipo espectral C-H na região azul do espectro.

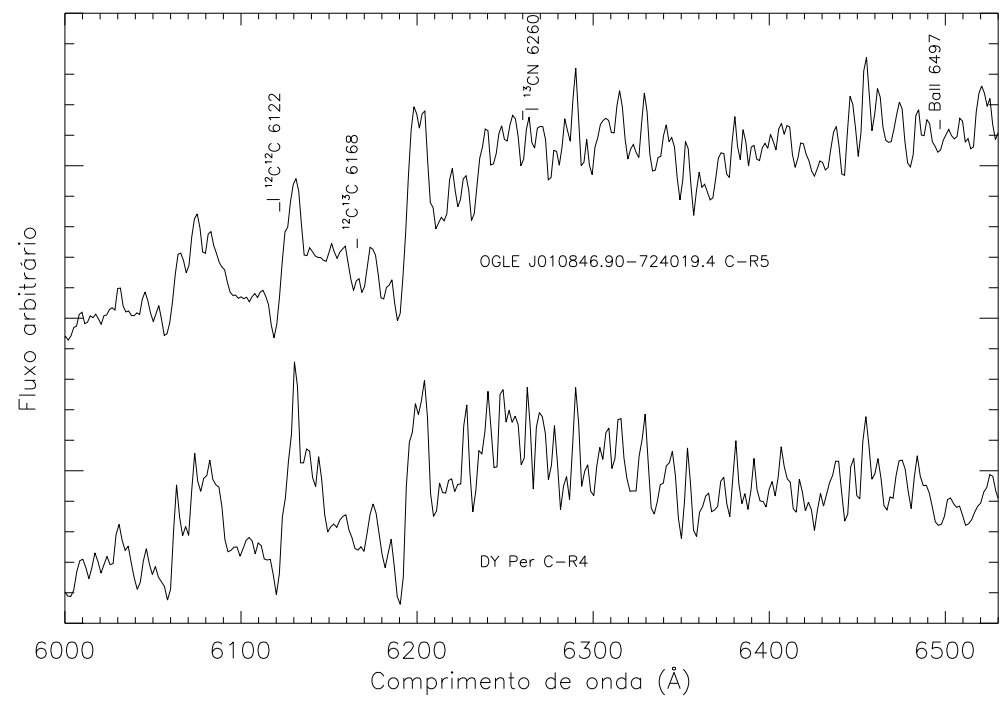

Figura 3.4: Detalhes das semelhanças entre objetos de tipo espectral C-R na região vermelha do espectro.

tométrica, pode estar associado ao seu estágio evolutivo, o que faz com que as temperaturas de suas atmosferas sejam relativamente superiores as das outras estrelas. 


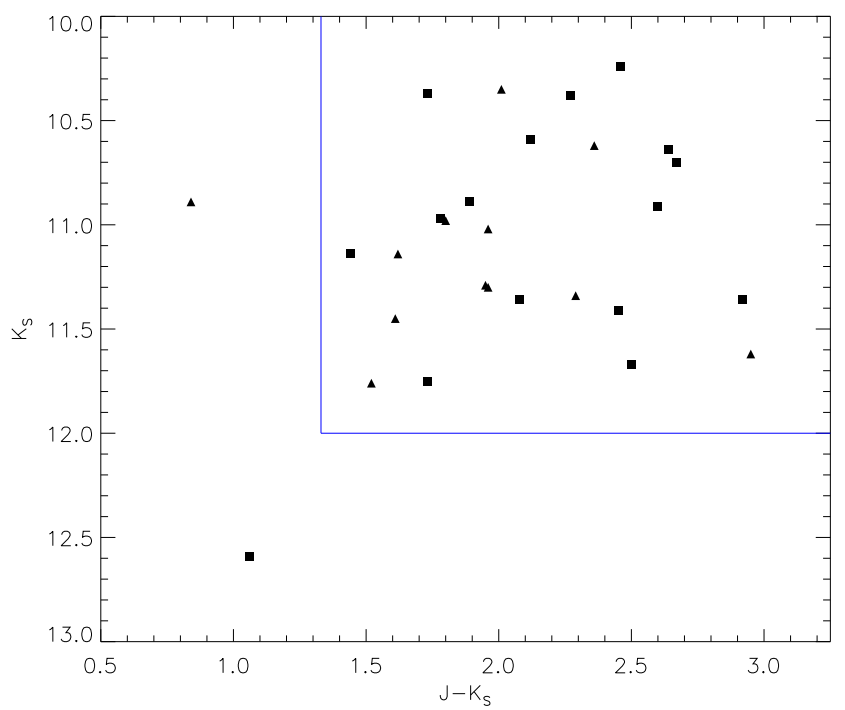

Figura 3.5: Diagrama cor-magnitude $\mathrm{J}-\mathrm{K}_{s}$ vs. $\mathrm{K}_{s}$. Triângulos representam objetos presentes na SMC e quadrados indicam objetos da LMC. As linhas azuis representam os limites da região onde residem as estrelas C $\left(\mathrm{J}-\mathrm{K}_{s}>1,33\right.$ e $\left.\mathrm{K}_{s}<12\right)$.

\subsection{Resultados}

\subsubsection{Tipos espectrais}

Como mostra a coluna 7 da Tabela 2.3, a amostra é composta em sua maioria por estrelas C-N. Como já visto anteriormente, esse tipo de estrela é caracterizado por espectros com abundâncias de elementos $s$ acima da solar. A sobreabundância desse tipo elemental é típica de estrelas em estágios avançados, onde os elementos primários presentes em seu interior tiveram tempo suficiente para passarem por processos de enriquecimentos de nêutrons.

Há cinco estrelas cujos espectros não se enquadram na classificação de MK93: OGLE J004032.22-732048.3 (tipo espectral dC), OGLE J051609.74-693517.9, OGLE J051810.88692626.5 (ambas com espectros compostos), OGLE J053910.32-695916.7 (M8 III) e OGLE J005401.40-731029.8 (da qual o tipo espectral ainda é incerto).

A gigante de tipo M8, OGLE J053910.32-695916.7, teve seu tipo espectral determinado pela identificação das fortes bandas de TiO. Sua classificação seguiu a comparação visual com espectros de Montes et al. (1997) e Gray e Corbally (2009).

O tipo espectral dC atribuído à estrela OGLE J004032.22-732048.3 ainda é duvidoso. 


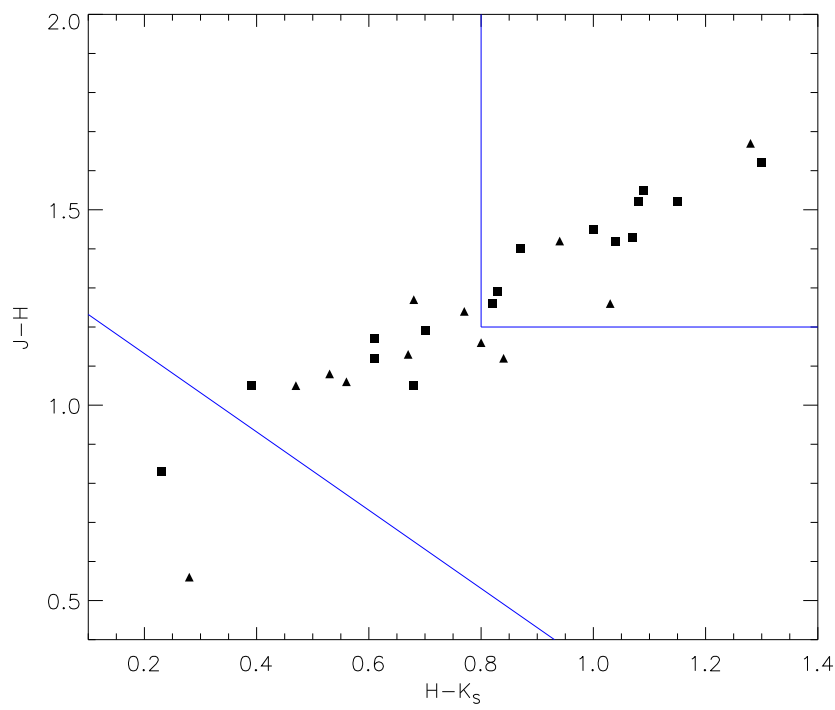

Figura 3.6: Diagrama cor-cor $\mathrm{H}-\mathrm{K}_{s}$ vs. J-H. Os símbolos são os mesmos da Fig. 3.5. A linha diagonal indica $\mathrm{J}-\mathrm{K}_{s}>1,33$; a região delimitada pelo quadrado azul, no canto superior direito do diagrama (H-K $>0,8$ e $\mathrm{J}-\mathrm{H}>1,2)$, indica os objetos obscurecidos em fase AGB

O espectro dessa estrela é distinto do padrão que segue o restante da amostra; não obstante, seu espectro é semelhante ao de estrelas dC apresentados por Gray e Corbally (2009). Embora a identificação de gigantes $\mathrm{C}$ tenha respaldo em critérios espectrais bem estabelecidos, não é raro estrelas C gigantes apresentarem espectros semelhantes aos de dC. Para garantir a classificação correta de uma estrela dC é necessária a busca por um critério que discrimine claramente as classes de luminosidade de estrelas C, tarefa até então pouco desenvolvida (Gray e Corbally, 2009). A peculiaridade do espectro desse objeto pode estar associada a possível binaridade, assim como ocorre com a estrela dC G77-61 (Dahn et al., 1977).

A estrela OGLE J005401.40-731029.8 (tipo espectral Ba?) possui espectro semelhante ao de estrelas de tipo tardio. Seu espectro se distingue do restante da amostra principalmente por não possuir bandas de moléculas de carbono tão fortes quanto as outras estrelas. Comparando-o aos de estrelas de Ba de Barnbaum et al. (1996), seria possível associar OGLE J005401.40-731029.8 a esse mesmo tipo espectral se as linhas desse elemento fossem mais fortes, o que é esperado para esse tipo espectral. Estudos observacionais de velocidades radiais de estrelas de Ba sugerem que todas elas são sistemas binários (McClure et al., 1980; McClure e Woodsworth, 1990; Jorissen e Mayor, 1988; Husti et al., 2009); 


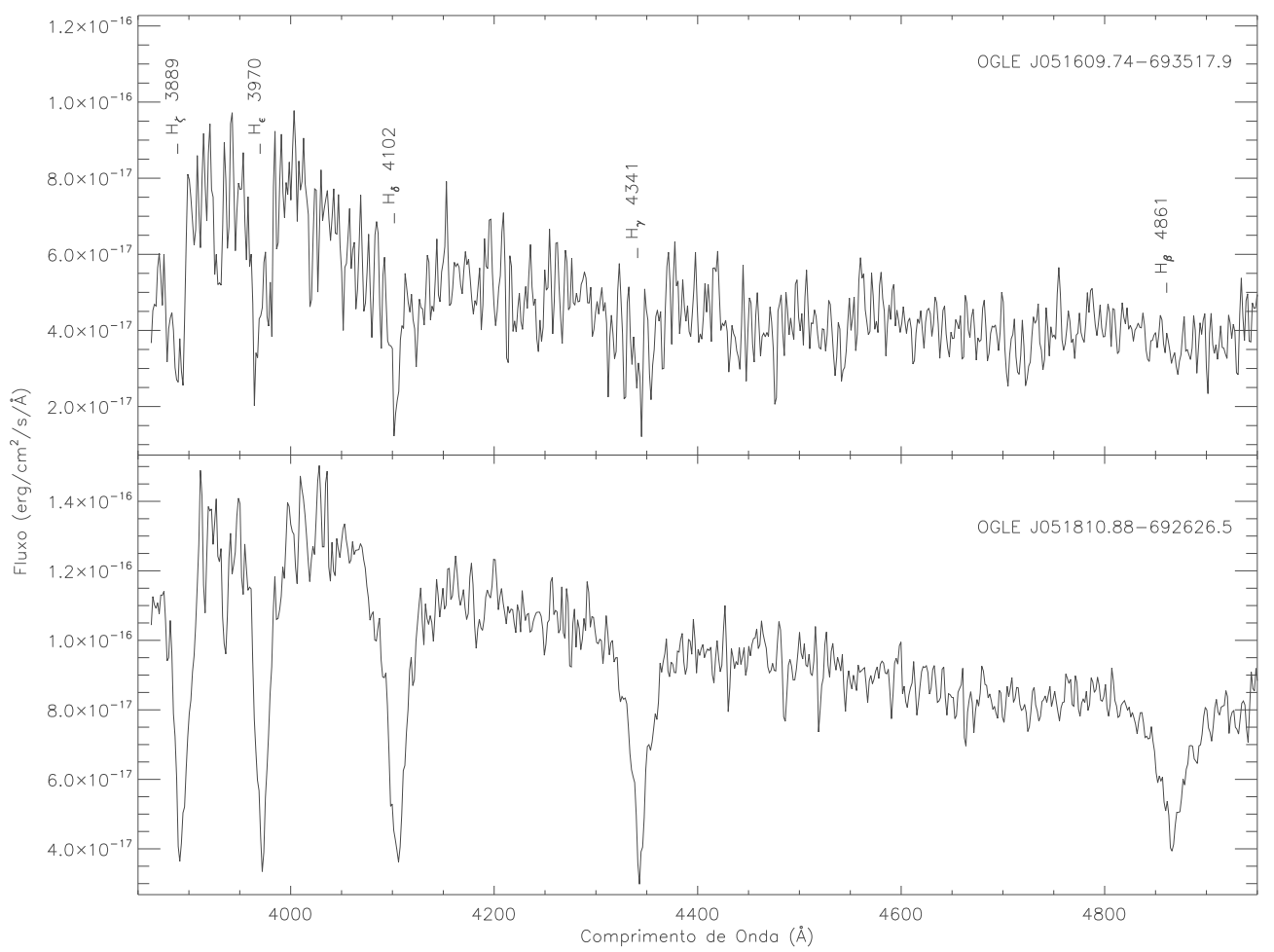

Figura 3.7: Destaque da região azul dos espectros dos sistemas binários OGLE J051609.74-693517.9 e OGLE J051810.88-692626.5

nesse sentido, a confirmação de seu tipo espectral possibilitará estudá-la como uma forte candidata a CEMP, assim como os outros objetos binários.

Os dois espectros compostos são típicos de sistemas binários.

\subsubsection{Binaridade}

As estrelas OGLE J051609.74-693517.9 e OGLE J051810.88-692626.5 (Fig. 2.3) possuem espectros nitidamente compostos: o lado azul é típico de uma estrela anã branca, com as linhas da série de Balmer apresentando asas alargadas (consequência da alta gravidade superficial), enquanto há um fluxo característico de estrelas frias no lado vermelho do espectro. As regiões entre 3850 e $4950 \AA$ dos dois espectros são destacadas na Figura 3.7 .

Há outro objeto com forte indício de pertencer a classe de sistemas binários. A estrela OGLE J053424.83-694400.1 possui tipo espectral C-H e, de acordo com McClure (1984), todas as estrelas desse tipo são na realidade sistemas binários, com troca de material 
enriquecido por elementos produzidos na fase AGB, da estrela gigante para a anã.

O objeto OGLE J004032.22-732048.3 (dC) possui velocidade radial $\mathrm{v}_{\text {rad }}=6,4 \mathrm{~km} / \mathrm{s}$, atípica para uma estrela pertencente à SMC. Logo, é possível que essa estrela pertença ao halo Galáctico. O estudo da variabilidade de sua velocidade radial passa a ser fundamental para se verificar a qual sistema pertence, bem como para a melhor compreensão de sua natureza peculiar.

O objeto OGLE J005401.40-731029.8 (Ba?), como já dito anteriormente, também possui chances de ser um objeto binário, portanto requer um estudo aprofundado acerca de sua natureza.

Portanto, há na amostra ao menos cinco objetos que, por serem potenciais estrelas binárias, possuem perfil de interesse no estudo de estrelas CEMP. Esses objetos terão fundamental contribuição na investigação dos efeitos da binaridade na composição química da atmosfera estelar, no que diz respeito tanto à deficiência em metais quanto à sobreabundância de carbono. Como os espectros dos outros objetos da amostra ou não permitem a identificação de binaridade ou não pertencem a classes em que tal característica esteja bem estabelecida, a futura associação desses objetos a alguma classe de sistemas binários requer o monitoramento da variação de velocidade radial ao longo de dias, informação não disponível para esses objetos até o momento.

\subsubsection{Fotometria}

A fotometria confirma o objeto OGLE J004032.22-732048.3 (dC) como peculiar. É importante apontar para a informação que diz respeito ao critério de identificação de estrelas dC apresentado por Westerlund et al. (1995), J-H $<0,75$ e $\mathrm{H}-\mathrm{K}>0,25$. A estrela possui $\mathrm{J}-\mathrm{H}=0,83$ e $\mathrm{H}-\mathrm{K}_{s}=0,23$, muito próximo do limite do intervalo estabelecido por esse critério, apesar de suas cores estarem fora desse intervalo. Essa evidência se apresenta como um ponto a favor da hipótese de ser um objeto dC.

O objeto C-H, uma estrela binária (como consequência da classificação espectral), também possui justificativa para se afastar da região de estrelas $\mathrm{C}$ em geral. Isso porque, como consequência da suposta binaridade, a composição química do objeto pode ter sido alterada devido a transferência de material enriquecido em elementos pesados de uma estrela para outra, fenômeno que pode influenciar a distribuição de energia e refletir na 
alteração de suas cores.

Os dois objetos que possuem espectros compostos, OGLE J051609.74-693517.9 e OGLE J051810.88-692626.5, são estrelas binárias localizadas em regiões de estrelas C (Fig. 3.5). Apesar da peculiaridade espectral, tais estrelas não se distinguem do comportamento fotométrico geral da amostra. Essa é uma evidência de que as estrelas C, enquanto classe espectral, possuem alguma relação, mesmo que fraca, com estrelas binárias, embora não seja possível apontar uma causalidade nessa relação. É mais um incentivo ao estudo desse tipo de vínculo.

Como pode ser verificado nas colunas 4 e 5 da Tabela 2.3 e sabendo que estrelas C possuem $-5,0<\mathrm{M}_{b o l}<-3,0$ (Westerlund et al., 1991), todas estrelas da amostra correspondem a esse critério. Logo, a análise das magnitudes bolométricas não aponta nenhuma inomogeneidade na amostra, apesar de outras informações levarem a conclusões no sentido oposto.

\subsubsection{Curvas de luz}

A grande maioria dos objetos apresenta curvas de luz com períodos típicos de Miras (de 80 a 1000 dias). Todavia há algumas curvas que não seguem esse padrão e portanto foram analisadas com mais atenção.

A curva de luz do objeto OGLE J053424.83-694400.1 (C-H3, Figura 2.4) possui quatro mínimos, sendo o primeiro deles 1,5 magnitudes menos brilhante do que os outros três. A aparente periodicidade de seus mínimos insinua a possibilidade de ser um sistema binário visual. No entanto, a investigação da periodicidade dos mínimos de luminosidade, separados em 336, 422 e 356 dias, mostra uma irregularidade que exclui tal hipótese. A menor diferença entre esses intervalos de mínimo de luminosidade é de 20 dias. Considerando um desvio de $3 \sigma$ da incerteza média da magnitude $\left(\sigma_{I}=0,01\right.$, Seção 2.4), a incerteza média do dia em que ocorre o mínimo é de \pm 7 dias. Mesmo considerando a possibilidade de ocorrer um erro dessa ordem nas magnitudes, influenciando um deslocamento dos mínimos das curvas de luz em relação ao seus valores verdadeiros, esse deslocamento seria insuficiente para provocar uma diferença que justificaria uma curva de luz característica de uma binária visual. Logo, a hipótese de binária (ao menos) visual não parece ser sustentada pela análise da curva de luz do objeto em questão, apesar de se esperar que, de acordo com seu tipo 
espectral C-H, tal estrela seja binária.

\subsubsection{Linhas de emissão em $H \alpha$}

Dentre as estrelas na amostra que pertencem a SMC, há duas com linhas de H $\alpha$ emissão, enquanto na LMC há 10 estrelas com essa propriedade.

A emissão em estrelas C é um fenômeno com estudos ainda inconclusivos. Meyssonnier e Azzopardi (1993), em seu levantamento de objetos com linhas de emissão em H $\alpha$ na SMC, apontaram para algumas estrelas de tipo tardio como Miras e simbióticas. Barnbaum (1994) cita autores que justificam tal emissão pela presença de atividade cromosférica. Nesses dois trabalhos, mostrou-se que a intensidade da emissão varia com tempo, indicando a existência de algum vínculo entre essas duas quantidades. Mikulasek e Graf (2005) constatam que todas as estrelas C que são ao mesmo tempo Miras possuem emissão em $\mathrm{H} \alpha$ e concluem que há correlação entre a variabilidade na magnitude $\mathrm{V}$ com a da linhas de emissão.

Uma análise qualitativa das curvas de luz dos objetos da amostra que possuem emissão em $\mathrm{H} \alpha$ em seus espectros mostra que há períodos de centenas de dias dentro das variações de luminosidade, o que é compatível com o intervalo de períodos aceitável para Miras. 


\section{Capítulo 4}

\section{Conclusões e perspectivas}

Neste projeto de pesquisa buscou-se informações sobre uma amostra contendo 28 estrelas ricas em carbono encontradas nas Nuvens de Magalhães, a partir de dados espectroscópicos previamente obtidos com o telescópio Magellan Clay e da fotometria obtida da literatura.

A caracterização da amostra e a classificação espectral de suas estrelas foi apreciada. Para tanto, recorreu-se a catálogos de espectros e a critérios fotométricos sobre os quais se sustentou a classificação.

Infelizmente, não foi possível a determinação de parâmetros atmosféricos, uma vez que a grade de modelos sintéticos não inclui ainda estrelas suficientemente frias, como as da amostra aqui estudadas.

A maioria dos objetos da amostra possuem espectros que se assemelham a estrelas C. Essas estrelas foram classificadas a partir da comparação visual com catálogos disponíveis na literatura. Alguns desses objetos são fortes candidatos a sistemas binários, como os classificados como dC e C-H. Logo, são indicados ao estudo do enriquecimento de carbono em estrelas CEMP e podem ajudar a desvendar os processos responsáveis pela sobreabundância desse elemento. Nesse sentido, fornecerão condições para testar a hipótese de transferência de material de uma estrela evoluída para outra relativamente jovem.

Foram estudadas as particularidades de alguns dos objetos, como variabilidade, linhas de emissão e potencial binaridade. O objeto OGLE J004032.22-732048.3, classificado como estrela dC, é de especial interesse para o estudo de estrelas CEMP uma vez que apresenta características peculiares tanto do ponto de vista fotométrico quanto espectroscópico. O objeto OGLE J005401.40-731029.8 é uma potencial estrela de bário, logo também se apre- 
senta como candidato a um estudo detalhado para melhor compreensão de sua natureza.

A descoberta de alguma relação entre estrelas CEMP e C trará luz ao problema do enriquecimento em carbono notado nas atmosferas estelares de estrelas da MW, ainda não esclarecidos. As informações reunidas acerca da amostra permitirão o estudo aprofundado sobre a origem da sobreabundância do carbono em estrelas pobres em metais. Para tanto, será necessária a determinação dos parâmetros físicos das estrelas reunidas a partir de métodos ainda em desenvolvimento. A partir desse resultado, será possível a busca por indícios da correlação entre o conteúdo metálico estelar deduzido para a Via Láctea e os indíces metálicos obtidos para as Nuvens de Magalhães. O estudo detalhado sobre a história de formação estelar dos objetos das Nuvens vinculará a evolução dessas duas galáxias-satélites à história da Via Láctea. 


\section{Referências Bibliográficas}

Alcock C., Allsman R. A., Alves D. R., et al., The MACHO Project: Microlensing Results from 5.7 Years of Large Magellanic Cloud Observations, ApJ, 2000, vol. 542, p. 281

Alksnis A., Balklavs A., Dzervitis U., Eglitis I., Absolute magnitudes of carbon stars from HIPPARCOS parallaxes, A\&A, 1998, vol. 338, p. 209

Alonso A., Arribas S., Martinez-Roger C., Broad band JHK infrared photometry of an extended sample of late type dwarfs and subdwarfs, A\&AS, 1994, vol. 107, p. 365

Alonso A., Arribas S., Martinez-Roger C., The empirical scale of temperatures of the low main sequence (F0V-K5V), A\&A, 1996, vol. 313, p. 873

Aoki W., Beers T. C., Christlieb N., Norris J. E., Ryan S. G., Tsangarides S., Carbonenhanced Metal-poor Stars. I. Chemical Compositions of 26 Stars, ApJ, 2007, vol. 655, p. 492

Aoki W., Beers T. C., Sivarani T., Marsteller B., Lee Y. S., Honda S., Norris J. E., Ryan S. G., Carollo D., Carbon-Enhanced Metal-Poor Stars. III. Main-Sequence Turnoff Stars from the SDSS SEGUE Sample, ApJ, 2008, vol. 678, p. 1351

Aoki W., Frebel A., Christlieb N., HE 1327-2326, an Unevolved Star with $[\mathrm{Fe} / \mathrm{H}]<-5.0$. I. A Comprehensive Abundance Analysis, ApJ, 2006, vol. 639, p. 897

Arce H. G., Goodman A. A., Measuring Galactic Extinction: A Test, ApJ, 1999, vol. 512, p. 135

Arribas S., Roger C. M., Infrared observations of metal-deficient stars, A\&AS, 1987, vol. 70, p. 303 
Baade W., The Resolution of Messier 32, NGC 205, and the Central Region of the Andromeda Nebula., ApJ, 1944, vol. 100, p. 137

Barnbaum C., A high-resolution spectral atlas of carbon stars, ApJS, 1994, vol. 90, p. 317

Barnbaum C., Stone R. P. S., Keenan P. C., A Moderate-Resolution Spectral Atlas of Carbon Stars: R, J, N, CH, and Barium Stars, ApJS, 1996, vol. 105, p. 419

Beers T. C., Christlieb N., The Discovery and Analysis of Very Metal-Poor Stars in the Galaxy, ARA\&A, 2005, vol. 43, p. 531

Beers T. C., Preston G. W., Shectman S. A., A search for stars of very low metal abundance. I, AJ, 1985, vol. 90, p. 2089

Beers T. C., Preston G. W., Shectman S. A., A search for stars of very low metal abundance. II, AJ, 1992, vol. 103, p. 1987

Bekki K., Chiba M., The Magellanic squall: gas replenishment from the Small to the Large Magellanic Cloud, MNRAS, 2007, vol. 381, p. 16

Bekki K., Stanimirovic S., The total mass and dark halo properties of the Small Magellanic Cloud, MNRAS, 2009, vol. 395, p. 342

Bessell M. S., Brett J. M., JHKLM photometry - Standard systems, passbands, and intrinsic colors, PASP, 1988, vol. 100, p. 1134

Blanco V. M., Blanco B. M., McCarthy M. F., Carbon and late M-type stars in the Magellanic Clouds, ApJ, 1980, vol. 242, p. 938

Blanco V. M., McCarthy M. F., The distribution of carbon and M-type giants in the Magellanic Clouds, AJ, 1983, vol. 88, p. 1442

Blanco V. M., McCarthy M. F., Identification of 849 cool-carbon LMC stars, AJ, 1990, vol. 100 , p. 674

Bond H. E., Where is Population III, ApJ, 1981, vol. 248, p. 606 
Bonifacio P., Monai S., Beers T. C., A Search for Stars of Very Low Metal Abundance. V. Photoelectric UBV Photometry of Metal-weak Candidates from the Northern HK Survey, AJ, 2000, vol. 120, p. 2065

Bromm V., Coppi P. S., Larson R. B., Forming the First Stars in the Universe: The Fragmentation of Primordial Gas, ApJ, 1999, vol. 527, p. 5

Bromm V., Coppi P. S., Larson R. B., The Formation of the First Stars. I. The Primordial Star-forming Cloud, ApJ, 2002, vol. 564, p. 23

Bromm V., Larson R. B., The First Stars, ARA\&A, 2004, vol. 42, p. 79

Burbidge E. M., Burbidge G. R., Fowler W. A., Hoyle F., Synthesis of the Elements in Stars, Reviews of Modern Physics, 1957, vol. 29, p. 547

Burstein D., Heiles C., H I, galaxy counts, and reddening - Variation in the gas-to-dust ratio, the extinction at high galactic latitudes, and a new method for determining galactic reddening, ApJ, 1978, vol. 225, p. 40

Burstein D., Heiles C., Reddenings derived from H I and galaxy counts - Accuracy and maps, AJ, 1982, vol. 87, p. 1165

Cannon A. J., Pickering E. C., The Henry Draper catalogue 0h, 1h, 2h, and 3h, Annals of Harvard College Observatory, 1918, vol. 91, p. 1

Carlson L. R., Sabbi E., Sirianni M., Hora J. L., Nota A., Meixner M., Gallagher J. S., Oey M. S., Pasquali A., Smith L. J., Tosi M., Walterbos R., Progressive Star Formation in the Young SMC Cluster NGC 602, ApJ, 2007, vol. 665, p. 109

Carr B., Cosmology - where is Population-III, Nature, 1987, vol. 326, p. 829

Carrera R., Gallart C., Aparicio A., Costa E., Mendez R. A., Noel N. E. D., The Chemical Enrichment History of the Small Magellanic Cloud and its Gradients, AJ, 2008, vol. 136, p. 1039

Castelli F., Kurucz R. L., New Grids of ATLAS9 Model Atmospheres, astro-ph/0405087, 2004 
Cayrel R., Depagne E., Spite M., Hill V., Spite F., François P., Plez B., Beers T., Primas F., Andersen J., Barbuy B., Bonifacio P., Molaro P., Nordstrom B., First stars V Abundance patterns from $\mathrm{C}$ to $\mathrm{Zn}$ and supernova yields in the early Galaxy, A\&A, 2004, vol. 416, p. 1117

Chiosi E., Vallenari A., Held E. V., Rizzi L., Moretti A., Age distribution of young clusters and field stars in the Small Magellanic Cloud, A\&A, 2006, vol. 452, p. 179

Christlieb N., Finding the Most Metal-poor Stars of the Galactic Halo with the Hamburg/ESO Objective-prism Survey (With 6 Figures), vol. 16 of Reviews in Modern Astronomy, Wiley, New York, 2003, p. 191

Christlieb N., Bessell M. S., Beers T. C., Gustafsson B., Korn A., Barklem P. S., Karlsson T., Mizuno-Wiedner M., Rossi S., A stellar relic from the early Milky Way, Nature, 2002, vol. 419, p. 904

Christlieb N., Green P. J., Wisotzki L., Reimers D., The stellar content of the Hamburg/ESO survey II. A large, homogeneously-selected sample of high latitude carbon stars, A\&A, 2001, vol. 375, p. 366

Christlieb N., Gustafsson B., Korn A. J., Barklem P. S., Beers T. C., Bessell M. S., Karlsson T., Mizuno-Wiedner M., HE 0107-5240, a Chemically Ancient Star. I. A Detailed Abundance Analysis, ApJ, 2004, vol. 603, p. 708

Cioni M. L., Blommaert J. A. D. L., Groenewegen M. A. T., Habing H. J., Hron J., Kerschbaum F., Loup C., Omont A., van Loon J. T., Whitelock P. A., Zijlstra A. A., Long period variables detected by ISO in the Small Magellanic Cloud, A\&A, 2003, vol. 406 , p. 51

Cioni M. L., van der Marel R. P., Loup C., Habing H. J., The tip of the red giant branch and distance of the Magellanic Clouds: results from the DENIS survey, A\&A, 2000, vol. 359 , p. 601

Clayton G. C., Cardelli J. A., Polarization and the ratio of total-to-selective extinction, AJ, 1988, vol. 96, p. 695 
Cohen J. G., Shectman S., Thompson I., McWilliam A., Christlieb N., Melendez J., Zickgraf F. J., Ramirez S., Swenson A., The Frequency of Carbon Stars among Extremely Metal-poor Stars, ApJ, 2005, vol. 633, p. 109

Cohen M., Wheaton W. A., Megeath S. T., Spectral Irradiance Calibration in the Infrared. XIV. The Absolute Calibration of 2MASS, AJ, 2003, vol. 126, p. 1090

Dahn C. C., Liebert J., Kron R. G., Spinrad H., Hintzen P. M., G77-61 - A dwarf carbon star, ApJ, 1977, vol. 216, p. 757

de Vaucouleurs G., de Vaucouleurs A., Corwin J. R., Second reference catalogue of bright galaxies , University of Texas Press, 1976

de Vaucouleurs G., Freeman K., Structure and dynamics of barred spiral galaxies, in particular of the Magellanic type, Vistas in Astronomy, 1972, vol. 14, p. 163

Dean C. A., Kinematic properties of the carbon stars, AJ, 1976, vol. 81, p. 364

Demers S., Irwin M. J., Kunkel W. E., Very red stars between the Magellanic Clouds Discovery of carbon stars in the outer LMC and SMC haloes, MNRAS, 1993, vol. 260, p. 103

Dieter N. H., Neutral hydrogen near the galactic poles, AJ, 1965, vol. 70, p. 552

Dolphin A. E., Walker A. R., Hodge P. W., Mateo M., Olszewski E. W., Schommer R. A., Suntzeff N. B., Old Stellar Populations of the Small Magellanic Cloud, ApJ, 2001, vol. 562, p. 303

Dopita M. A., Lawrence C. J., Ford H. C., Webster B. L., The kinematics and internal dynamics of planetary nebulae in the small Magellanic Cloud, ApJ, 1985, vol. 296, p. 390

Downes R. A., Margon B., Anderson S. F., Harris H. C., Knapp G. R., Schroeder J., Schneider D. P., York D. G., Pier J. R., Brinkmann J., Faint High-Latitude Carbon Stars Discovered by the Sloan Digital Sky Survey: An Initial Catalog, AJ, 2004, vol. 127, p. 2838 
Feast M., The Distance to the Large Magellanic Cloud; A Critical Review. In New Views of the Magellanic Clouds, vol. 190, 1999, p. 542

François P., Depagne E., Hill V., Spite M., Spite F., Plez B., Beers T. C., Andersen J., James G., Barbuy B., Cayrel R., Bonifacio P., Molaro P., Nordstrom B., Primas F., First stars. VIII. Enrichment of the neutron-capture elements in the early Galaxy, A\&A, 2007, vol. 476 , p. 935

Frebel A., Aoki W., Christlieb N., Nucleosynthetic signatures of the first stars, Nature, 2005, vol. 434, p. 871

Frebel A., Christlieb N., Norris J. E., Beers T. C., Bessell M. S., Rhee J., Fechner C., Marsteller B., Rossi S., Thom C., Wisotzki L., Reimers D., Bright Metal-poor Stars from the Hamburg/ESO Survey. I. Selection and Follow-up Observations from 329 Fields, ApJ, 2006, vol. 652, p. 1585

Freedman W. L., Madore B. F., Gibson B. K., Ferrarese L., Kelson D. D., Sakai S., Mould J. R., Kennicutt R. C., Ford H. C., Graham J. A., Huchra J. P., Hughes S. M. G., Illingworth G. D., Macri L. M., Stetson P. B., Final Results from the Hubble Space Telescope Key Project to Measure the Hubble Constant, ApJ, 2001, vol. 553, p. 47

Frogel J. A., Persson S. E., Matthews K., Aaronson M., Photometric studies of composite stellar systems. I - CO and JHK observations of E and S0 galaxies, ApJ, 1978, vol. 220, p. 75

Fujimoto M., Sofue Y., Dynamical evolution of the triple system of the Galaxy, the Large and Small Magellanic Clouds, A\&A, 1976, vol. 47, p. 263

Gibson B. K., The distance to the Large Magellanic Cloud., Memorie della Societa Astronomica Italiana, 2000, vol. 71, p. 693

Gray R. O., Corbally C., Stellar Spectral Classification. Princeton University Press, 2009, 592

Grevesse N., Asplund M., Sauval A. J., The Solar Chemical Composition, Space Science Reviews, 2007, vol. 130, p. 105 
Gustafsson B., Edvardsson B., Eriksson K., Jörgensen U. G., Mizuno-Wiedner M., Plez B., A New MARCS Grid. In Modelling of Stellar Atmospheres , vol. 210 of IAU Symposium, 2003, p. $4 \mathrm{P}$

Gustafsson B., Edvardsson B., Eriksson K., Jörgensen U. G., Nordlund A., Plez B., A grid of MARCS model atmospheres for late-type stars. I. Methods and general properties, A\&A, 2008, vol. 486, p. 951

Harris J., Zaritsky D., The Star Formation History of the Small Magellanic Cloud, AJ, 2004, vol. 127 , p. 1531

Hatzidimitriou D., Croke B. F., Morgan D. H., Cannon R. D., Kinematics of carbon stars in the outer regions of the Small Magellanic Cloud, A\&AS, 1997, vol. 122, p. 507

Heiles C., Jenkins E. B., An almost complete survey of 21-cm line radiation for galactic latitudes of $10 \mathrm{deg}$ and higher. V - Photographic presentation and qualitative comparison with other data, A\&A, 1976, vol. 46, p. 333

Hernandez X., Ferrara A., Cosmological origin of the lowest metallicity halo stars, MNRAS, 2001, vol. 324, p. 484

Herwig F., Evolution of Asymptotic Giant Branch Stars, ARA\&A, 2005, vol. 43, p. 435

Hilditch R. W., Howarth I. D., Harries T. J., Forty eclipsing binaries in the Small Magellanic Cloud: fundamental parameters and Cloud distance, MNRAS, 2005, vol. 357, p. 304

Hill V., Barbuy B., Spite M., Spite F., Cayrel R., Plez B., Beers T. C., Nordström B., Nissen P. E., Heavy-element abundances in the CH/CN-strong very metal-poor stars CS 22948-27 and CS 29497-34, A\&A, 2000, vol. 353, p. 557

Hughes S. M. G., Wood P. R., Reid N., Long-period variables in the Large Magellanic Cloud. III - Evidence of a kinematic spheroidal population, AJ, 1991, vol. 101, p. 1304

Husti L., Gallino R., Bisterzo S., Straniero O., Cristallo S., Barium Stars: Theoretical Interpretation, Publications of the Astronomical Society of Australia, 2009, vol. 26, p. 176 
Johnson H. L., , 1963, Basic Astronomical Data: Stars and stellar systems, edited by K. A. Strand. Published by the University of Chicago Press, Chicago, IL USA, p.204 p. 204

Johnson H. L., Morgan W. W., Fundamental stellar photometry for standards of spectral type on the revised system of the Yerkes spectral atlas, ApJ, 1953, vol. 117, p. 313

Jorissen A., Mayor M., Radial velocity monitoring of a sample of barium and S stars using CORAVEL - Towards an evolutionary link between barium and S stars, A\&A, 1988, vol. 198 , p. 187

Kallivayalil N., van der Marel R. P., Alcock C., Is the SMC Bound to the LMC? The Hubble Space Telescope Proper Motion of the SMC, ApJ, 2006, vol. 652, p. 1213

Keenan P. C., Revised MK spectral classification of the red carbon stars, PASP, 1993, vol. 105 , p. 905

Keenan P. C., Morgan W. W., The Classification of the Red Carbon Stars., ApJ, 1941, vol. 94 , p. 501

Kontizas E., Dapergolas A., Morgan D. H., Kontizas M., A Catalogue of carbon stars in the LMC, A\&A, 2001, vol. 369, p. 932

Kurucz R., Diatomic Molecular Data for Opacity Calculations., Diatomic Molecular Data for Opacity Calculations. Kurucz CD-ROM No. 15. Cambridge, Mass.: Smithsonian Astrophysical Observatory, 1993., 1993, vol. 15

Landsman W. B., The IDL Astronomy User's Library, vol. 52, 1993, p. 246

Lequeux J., Interstellar Dust in the Magellanic Clouds - Extinction and Far-Infrared Emission. In Recent Developments of Magellanic Cloud Research , 1989, p. 119

Lin D. N. C., Jones B. F., Klemola A. R., The motion of the Magellanic clouds, origin of the Magellanic Stream, and the mass of the Milky Way, ApJ, 1995, vol. 439, p. 652

Lodders K., Solar System Abundances and Condensation Temperatures of the Elements, ApJ, 2003, vol. 591, p. 1220 
Lucatello S., Beers T. C., Christlieb N., Barklem P. S., Rossi S., Marsteller B., Sivarani T., Lee Y. S., The Frequency of Carbon-enhanced Metal-poor Stars in the Galaxy from the HERES Sample, ApJ, 2006, vol. 652, p. 37

Lucatello S., Gratton R., Cohen J. G., Beers T. C., Christlieb N., Carretta E., Ramirez S., Stellar Archaeology: A Keck Pilot Program on Extremely Metal-Poor Stars From the Hamburg/ESO Survey. III. The Lead (Pb) Star HE 0024-2523, AJ, 2003, vol. 125, p. 875

Mackey J., Bromm V., Hernquist L., Three Epochs of Star Formation in the High-Redshift Universe, ApJ, 2003, vol. 586, p. 1

Marigo P., Girardi L., Stellar Population Synthesis Including Planetary Nebulae. In Planetary Nebulae as Astronomical Tools , vol. 804, AIP Conference Proceedings, 2005, p. 284

Masseron T., Johnson J. A., Plez B., Eck S. V., Primas F., Goriely S., Jorissen A., A holistic approach to carbon-enhanced metal-poor stars, arXiv:0901.4737, 2009

Masseron T., van Eck S., Famaey B., Goriely S., Plez B., Siess L., Beers T. C., Primas F., Jorissen A., CS 30322-023: an ultra metal-poor TP-AGB star?, A\&A, 2006, vol. 455, p. 1059

Mathewson D. S., Cleary M. N., Murray J. D., The Magellanic stream, ApJ, 1974, vol. 190, p. 291

McClure R. D., The binary nature of the CH stars, ApJ, 1984, vol. 280, p. 31

McClure R. D., Fletcher J. M., Nemec J. M., The binary nature of the barium stars, ApJ, 1980, vol. 238 , p. 35

McClure R. D., Woodsworth A. W., The binary nature of the barium and CH stars. III Orbital parameters, ApJ, 1990, vol. 352, p. 709

McWilliam A., Preston G. W., Sneden C., Searle L., Spectroscopic Analysis of 33 of the Most Metal Poor Stars. II., AJ, 1995, vol. 109, p. 2757 
Mermilliod J., Compilation of Eggen's UBV data, transformed to UBV (unpublished), Catalogue of Eggen's UBV data, 1986

Meyssonnier N., Azzopardi M., A New Catalogue of H alpha Emission Line Stars and Small Nebulae in the Small Magellanic Cloud, A\&AS, 1993, vol. 102, p. 451

Mikulasek Z., Graf T., Atlas of H alpha emission lines and V light curves of 30 carbon Miras, Contributions of the Astronomical Observatory Skalnate Pleso, 2005, vol. 35, p. 83

Montes D., Martin E. L., Fernandez-Figueroa M. J., Cornide M., de Castro E., Library of high and mid-resolution spectra in the CA II H \& K, Halpha, Hbeta NA i D1, D2, and He i D3 line regions of F, G, K and M field stars, A\&AS, 1997, vol. 123, p. 473

Morgan D. H., Hatzidimitriou D., A survey of carbon stars in the Small Magellanic Cloud., A\&AS, 1995, vol. 113, p. 539

Mori M., Ferrara A., Madau P., Early Metal Enrichment by Pregalactic Outflows. II. Three-dimensional Simulations of Blow-Away, ApJ, 2002, vol. 571, p. 40

Muller E., Stanimirovic S., Rosolowsky E., Staveley-Smith L., A Statistical Investigation of H I in the Magellanic Bridge, ApJ, 2004, vol. 616, p. 845

Nakamura F., Umemura M., On the Initial Mass Function of Population III Stars, ApJ, 2001, vol. 548, p. 19

Nakamura F., Umemura M., The Stellar Initial Mass Function in Primordial Galaxies, ApJ, 2002, vol. 569, p. 549

Nelder J. A., Mead R., A Simplex Method for Function Minimization, The Computer Journal, 1965, vol. 7, p. 308

Norris J. E., Christlieb N., Korn A. J., Eriksson K., Bessell M. S., Beers T. C., Wisotzki L., Reimers D., HE 0557-4840: Ultra-Metal-Poor and Carbon-Rich, ApJ, 2007, vol. 670, p. 774

Norris J. E., Ryan S. G., Beers T. C., Extremely Metal-poor Stars. The Carbon-rich, Neutron Capture Element-poor Object CS 22957-027, ApJ, 1997, vol. 489, p. 169 
Ochsenbein F., Bauer P., Marcout J., The VizieR database of astronomical catalogues, A\&AS, 2000, vol. 143, p. 23

Oey M. S., The number and metallicities of the most metal-poor stars, MNRAS, 2003, vol. 339 , p. 849

Omukai K., Protostellar Collapse with Various Metallicities, ApJ, 2000, vol. 534, p. 809

Omukai K., Palla F., On the Formation of Massive Primordial Stars, ApJ, 2001, vol. 561, p. 55

Paczynski B., Evolution of Single Stars. VI. Model Nuclei of Planetary Nebulae, Acta Astronomica, 1971, vol. 21, p. 417

Paczynski B., Gravitational microlensing by the galactic halo, ApJ, 1986, vol. 304, p. 1

Paczynski B., Gravitational microlensing of the Galactic bulge stars, ApJ, 1991, vol. 371, p. 63

Piatti A. E., Sarajedini A., Geisler D., Clark D., Seguel J., Young star clusters immersed in intermediate-age fields in the Small Magellanic Cloud, MNRAS, 2007, vol. 377, p. 300

Plez B., Cohen J. G., Analysis of the carbon-rich very metal-poor dwarf G77-61, A\&A, 2005, vol. 434, p. 1117

Putman M. E., Gibson B. K., Staveley-Smith L., et al., Tidal disruption of the Magellanic Clouds by the Milky Way, Nature, 1998, vol. 394, p. 752

Rafelski M., Zaritsky D., The Star Clusters of the Small Magellanic Cloud: Age Distribution, AJ, 2005, vol. 129, p. 2701

Raimondo G., Cioni M. L., Rejkuba M., Silva D. R., Pulsation properties of C stars in the Small Magellanic Cloud, A\&A, 2005, vol. 438, p. 521

Rebeirot E., Azzopardi M., Westerlund B. E., Carbon stars in the Small Magellanic Cloud. II - Catalogue of 1707 objects with identifications and spectrophotometry, A\&AS, 1993, vol. 97 , p. 603 
Rees M. J., Opacity-limited hierarchical fragmentation and the masses of protostars, MNRAS, 1976, vol. 176, p. 483

Reeves H., Fowler W. A., Hoyle F., Galactic Cosmic Ray Origin of Li, Be and B in Stars, Nature, 1970, vol. 226, p. 727

Reimers D., A wide-angle objective prism survey for bright quasars., The Messenger, 1990, vol. 60 , p. 13

Rieke G. H., Lebofsky M. J., The interstellar extinction law from 1 to 13 microns, ApJ, 1985, vol. 288 , p. 618

Rossi S., Beers T. C., Sneden C., Carbon Abundances for Metal-Poor Stars Based on Medium-Resolution Spectra. In The Third Stromlo Symposium: The Galactic Halo , vol. 165 of Astronomical Society of the Pacific Conference Series, 1999, p. 264

Russell S. C., Dopita M. A., Abundances of the heavy elements in the Magellanic Clouds. III - Interpretation of results, ApJ, 1992, vol. 384, p. 508

Ryan S. G., Norris J. E., Beers T. C., Extremely Metal-poor Stars. II. Elemental Abundances and the Early Chemical Enrichment of the Galaxy, ApJ, 1996, vol. 471, p. 254

Sanduleak N., Philip A. G. D., Carbon stars and late-type suspected supergiants in the LMC, Publications of the Warner \& Swasey Observatory, 1977, vol. 2, p. 105

Sanford R. F., Radial Velocities of 283 Stars of Spectral Classes R and N., ApJ, 1944, vol. 99 , p. 145

Schlegel D. J., Finkbeiner D. P., Davis M., Maps of Dust Infrared Emission for Use in Estimation of Reddening and Cosmic Microwave Background Radiation Foregrounds, ApJ, 1998, vol. 500, p. 525

Schneider R., Ferrara A., Natarajan P., Omukai K., First Stars, Very Massive Black Holes, and Metals, ApJ, 2002, vol. 571, p. 30

Schneider R., Ferrara A., Salvaterra R., Omukai K., Bromm V., Low-mass relics of early star formation, Nature, 2003, vol. 422, p. 869 
Sivarani T., Beers T. C., Lee Y. S., Kennedy C., Carollo D., Masseron T., Plez B., Norris J., Bessel M., Rossi S., Parameters and Kinematics of Carbon Enhanced Metal Poor (CEMP) Stars from the Hamburg/ESO Survey. In 211th Meeting of the American Astronomical Society w/ the Historical Astronomy Division \& the High Energy Astrophysics Division , 104.16, 2008

Skrutskie M. F., Cutri R. M., Stiening R., The Two Micron All Sky Survey (2MASS), AJ, 2006, vol. 131, p. 1163

Spite F., Element Abundances in the Magellanic Clouds - Part Two - Cool Stars. In Recent Developments of Magellanic Cloud Research , Observatoire de Paris, 1989, p. 37

Stanimirovic S., Staveley-Smith L., Jones P. A., A New Look at the Kinematics of Neutral Hydrogen in the Small Magellanic Cloud, ApJ, 2004, vol. 604, p. 176

Tonry J., Davis M., A survey of galaxy redshifts. I - Data reduction techniques, AJ, 1979, vol. 84 , p. 1511

Toomre A., R0 from Exponential Disk Models, Quarterly Journal of the Royal Astronomical Society, 1972, vol. 13, p. 241

Tornatore L., Ferrara A., Schneider R., Population III stars: hidden or disappeared?, MNRAS, 2007, vol. 382, p. 945

Tumlinson J., Carbon-enhanced Hyper-Metal-poor Stars and the Stellar IMF at Low Metallicity, ApJ, 2007, vol. 665, p. 1361

Turon C., Egret D., Gomez A., Grenon M., Jahreiss H., Requieme Y., Argue A. N., BecBorsenberger A., Dommanget J., Mennessier M. O., Arenou F., Chareton M., Crifo F., Mermilliod J. C., Morin D., Nicolet B., Nys O., Prevot L., Rousseau M., Perryman M. A. C., Hipparcos Input Catalogue, Version 2 (Turon+ 1993), VizieR Online Data Catalog, 1996, vol. 1196

Udalski A., The Optical Gravitational Lensing Experiment. Real Time Data Analysis Systems in the OGLE-III Survey, Acta Astronomica, 2003, vol. 53, p. 291 
Uehara H., Inutsuka S., Does Deuterium Enable the Formation of Primordial Brown Dwarfs?, ApJ, 2000, vol. 531, p. 91

Umeda H., Nomoto K., Nucleosynthesis of Zinc and Iron Peak Elements in Population III Type II Supernovae: Comparison with Abundances of Very Metal Poor Halo Stars, ApJ, 2002, vol. 565 , p. 385

Umeda H., Nomoto K., First-generation black-hole-forming supernovae and the metal abundance pattern of a very iron-poor star, Nature, 2003, vol. 422, p. 871

van der Marel R. P., Alves D. R., Hardy E., Suntzeff N. B., New Understanding of Large Magellanic Cloud Structure, Dynamics, and Orbit from Carbon Star Kinematics, AJ, 2002, vol. 124 , p. 2639

van Kuilenburg J., A Systematic Search for High-Velocity Hydrogen Outside the Galactic Plane II, A\&A, 1972, vol. 16, p. 276

Wallerstein G., Knapp G. R., Carbon Stars, ARA\&A, 1998, vol. 36, p. 369

Wang M., Chin Y., Henkel C., Whiteoak J. B., Cunningham M., Abundances and Isotope Ratios in the Magellanic Clouds: the Star-Forming Environment of N 113, ApJ, 2009, vol. 690 , p. 580

Wannier P., Wrixon G. T., An Unusual High-Velocity Hydrogen Feature, ApJ, 1972, vol. 173, p. L119

Westerlund B. E., Discovery of an S star in the Fornax dwarf elliptical galaxy, A\&A, 1983, vol. 118, p. L5

Westerlund B. E., Review: an Overview of the Structure and Kinematics of the Magellanic Clouds. In The Magellanic Clouds, vol. 148, International Astronomical Union. Symposium no. 148, 1991, p. 15

Westerlund B. E., The Magellanic Clouds. Cambridge Astrophysics Series, 1997

Westerlund B. E., Azzopardi M., Breysacher J., Rebeirot E., Faint carbon stars in the Small Magellanic Cloud. II., A\&A, 1995, vol. 303, p. 107 
Westerlund B. E., Azzopardi M., Rebeirot E., Breysacher J., The evolution of carbon stars in the Magellanic Clouds, A\&AS, 1991, vol. 91, p. 425

Westerlund B. E., Olander N., Richer H. B., Crabtree D. R., A catalogue of carbon stars in the Large Magellanic Cloud., A\&AS, 1978, vol. 31, p. 61

Wheeler J. C., Sneden C., Truran J. W., Abundance ratios as a function of metallicity, ARA\&A, 1989, vol. 27, p. 279

Wisotzki L., Christlieb N., Bade N., Beckmann V., Kohler T., Vanelle C., Reimers D., The Hamburg/ESO survey for bright QSOs. III. A large flux-limited sample of QSOs, A\&A, 2000, vol. 358, p. 77

Wood P. R., Bessell M. S., Fox M. W., Long-period variables in the Magellanic Clouds Supergiants, AGB stars, supernova precursors, planetary nebula precursors, and enrichment of the interstellar medium, ApJ, 1983, vol. 272, p. 99

Yamashita Y., The C-classification of the spectra of carbon stars., Annals of the Tokyo Astronomical Observatory, 1972, vol. 13, p. 169

Yoshii Y., Sabano Y., Fragmentation of Cosmic Gas Clouds due to Thermal Instabilities, PASJ, 1980, vol. 32, p. 229

Yoshizawa A. M., Noguchi M., The dynamical evolution and star formation history of the Small Magellanic Cloud: effects of interactions with the Galaxy and the Large Magellanic Cloud, MNRAS, 2003, vol. 339, p. 1135

Zagury F., Reddening law and interstellar dust properties along Magellanic sight-lines, Astrophysics and Space Science, 2007, vol. 312, p. 113

Zaritsky D., Harris J., Quantifying the Drivers of Star Formation on Galactic Scales. I. The Small Magellanic Cloud, ApJ, 2004, vol. 604, p. 167 